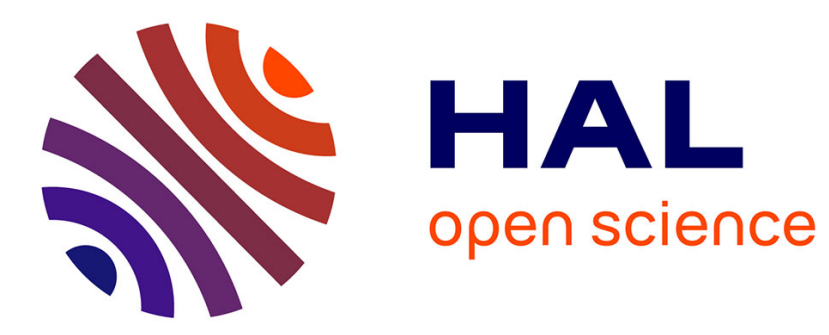

\title{
Sources historiques et produits locaux: fiscalité, techniques et production du papier dans le sud de l'Anhui
}

\author{
Michela Bussotti
}

\section{To cite this version:}

Michela Bussotti. Sources historiques et produits locaux : fiscalité, techniques et production du papier dans le sud de l'Anhui. Bulletin de l'Ecole française d'Extrême-Orient, 2014, 100 (1), pp.261-323. 10.3406/befeo.2014.6177 . halshs-01889987

\section{HAL Id: halshs-01889987 https://shs.hal.science/halshs-01889987}

Submitted on 8 Oct 2018

HAL is a multi-disciplinary open access archive for the deposit and dissemination of scientific research documents, whether they are published or not. The documents may come from teaching and research institutions in France or abroad, or from public or private research centers.
L'archive ouverte pluridisciplinaire HAL, est destinée au dépôt et à la diffusion de documents scientifiques de niveau recherche, publiés ou non, émanant des établissements d'enseignement et de recherche français ou étrangers, des laboratoires publics ou privés. 


\section{Sources historiques et produits locaux : fiscalité, techniques et} production du papier dans le sud de l'Anhui

Michela Bussotti

\section{Citer ce document / Cite this document :}

Bussotti Michela. Sources historiques et produits locaux : fiscalité, techniques et production du papier dans le sud de l'Anhui. In: Bulletin de l'Ecole française d'Extrême-Orient. Tome 100, 2014. pp. 261-323;

doi : https://doi.org/10.3406/befeo.2014.6177

https://www.persee.fr/doc/befeo_0336-1519_2014_num_100_1_6177

Fichier pdf généré le 08/05/2018 


\title{
Résumé
}

Cet article retrace la production du papier dans l'Anhui du Sud, sur la base des informations essentiellement tirées des monographies de l'administration, complétées par d'autres sources locales. La matière est organisée chronologiquement, en trois sections, correspondant à la dynastie des Song (960-1279), à la période Yuan-Ming (XIIle-XVIle s.), ainsi qu'à la dernière dynastie et à la période républicaine (du milieu du XVIle s. au milieu du XXe s.). On peut y voir comment la géographie de sa production se modifie au cours du temps, et en quoi le papier reste un produit économique important. Ce constat est valide, même si l'angle de notre regard change, en raison des transformations qui sont intervenues dans sa production et dans son utilisation, mais aussi sur la base de la nature des données à notre disposition, qui varient au cours des siècles. Quelques techniques de production sont également traitées dans les textes, ce qui est remarquable compte tenu de la relative rareté de ce type d'information.

\begin{abstract}
This article investigates the production of paper in southern Anhui, based on information mostly culled from administration monographs, supplemented with other local sources. The material is organized chronologically, in three sections, corresponding to the Song dynasty (960-1279), the Yuan-Ming period (13th-17th c.), and the last dynasty as well as the republican period (from the middle of the 17th c. to the middle of the 20th). This reveals how the geography of paper production changes over time, but also the stability of its importance as an economic product. This observation remains valid despite the fact that our perspective changes due to the transformations that occurred in its production and use, and due to the nature of the sources available to us, that vary from century to century. Some of the production techniques are discussed in the texts too, which is remarkable in view of the relative rarity of this type of information.
\end{abstract}




\title{
Sources historiques et produits locaux : fiscalité, techniques et production du papier dans le Sud de l'Anhui
}

\author{
Michela Bussottr *
}

\section{Introduction}

Lors de mes premières recherches sur l'histoire du papier ancien, j'avais cherché à savoir quels étaient les papiers utilisés dans les livres de luxe, dits de Hui 徽, imprimés vers 1600 dans cette région (Huizhou 徽州) du Sud de l'actuelle province de l'Anhui 安徽, ou dans d'autres centres urbains de la Chine centre-orientale (Cartes $1 \& 2)^{1}$. Or, sur ce point, les informations dans les textes locaux et d'époque sont rares. Cette façon de procéder fut certes dictée par le fait que le sujet était étudié dans le cadre de ma thèse de doctorat, mais elle suivait aussi une direction inspirée par des publications de référence : que ce soit dans le volume de Tsien Tsuen-Hsuin 錢存訓 dans l'encyclopédie Needham, ou dans celui de Pan Jixing 潘吉星 dans l'encyclopédie sur l'histoire des techniques chinoises ${ }^{2}$, le papier et l'imprimerie sont présentés ensemble. Le fait qu'il s'agisse de deux des «grandes inventions chinoises » a eu son poids dans l'association des thématiques. Mais il est aussi légitime de penser que cela s'est fait sous l'influence d'une vision européocentrée qui a, bon an mal an, dominé les travaux du $\mathrm{xx}^{\mathrm{e}}$ siècle,

\footnotetext{
* Écolc françaisc d'Extrême-Oricnt (EFEO) ct Centre d'ćtudes sur la Chinc moderne ct contemporaine (CEMC).

Je remercie Christian Lamouroux pour ses observations et les lectures qu'il m'a conseillées, notamment à propos de la dynastie des Song (960-1279); si des erreurs demeurent ici, elles sont entièrement de ma responsabilité. Mes remerciements vont aussi aux rapporteurs anonymes ainsi qu'aux collègues Caroline Bodolec, Frédéric Obringer et Françoise Sabban : c'est à l'occasion du séminaire « Techniques, objets élaborés et PCI » (EHESS-CECMC), pendant l'année 2013-2014, que j'ai repris mes recherches sur le papier.

1. Les recherches conduites pendant la première année de mes études doctorales ont fourni la matière à un chapitre de ma thèse sur les éditions illustrées des $x \mathrm{VI}^{\mathrm{e}}$ et $\mathrm{xVII}$ siècles de Huizhou, une ancienne préfecture dont le territoire correspond à la partie la plus méridionale de l'actuelle province de l'Anhui et à une petite portion du nord-est de la province voisine, le Jiangxi. Mais, à l'exclusion d'une traduction italienne partielle, ces matériaux sont restés inexploités. M. Bussotti, Gravures de l'école de Hui. Étude du livre illustré chinois de la fin du xvf siècle au milieu du XVIf siècle, thèse de doctorat, 1997, p. 30-53 et « Note sulla carta prodotta nel Sud dell'Anhui durante le dinastie Song e Yuan », dans Maurizio Scarpari \& Tiziana Lippiello (éd.), Scritti in onore di Lionello Lanciotti per l'ottantesimo compleanno, Venezia, Libreria Cafoscarina, 2005, p. 181-189.
}

2. Pan Jixing 潘吉星, Zaozhi yu yinshua juan 造紙與印刷卷, dans «Zhongguo kexue jishu shi » 中國科學技術史, Beijing, Kexue chubanshe, 1998 ; Tsien Tsuen-Hsuin, Paper and Printing, dans Joseph Needham (éd.), «Science and Civilisation in China », vol. 5, part 1, Cambridge, Cambridge University Press, 1985. 
y compris ceux qui se voulaient très sinophiles ${ }^{3}$. Il est tout à fait exact qu'en Occident ce binôme va de pair : le papier étant arrivé juste avant la " découverte » de l'imprimerie, de laquelle il est l'indispensable support, les deux techniques ainsi que les activités les concernant se développèrent presque au même moment ${ }^{4}$; en outre, papier et imprimerie représentent les deux éléments nécessaires à la rupture, sinon à la révolution, que la diffusion de l'imprimé a contribué à engendrer dans les systèmes culturels et sociaux des pays du continent européen.

Cependant, en Chine, le papier comptait déjà des siècles d'histoire, avant la diffusion de la xylographie dans un premier temps, et des caractères mobiles dans un second temps. Il était déjà le support de l'encre, pour les manuscrits et les estampages ; ces procédés de reproduction de textes et d'images vont d'ailleurs continuer à être utilisés en même temps que l'imprimé. La presse, elle, avec toutes les exigences qu'elle induit pour le papier à l'impression, mais aussi avec ses potentialités de mécanisation - qui en Europe allèrent de pair avec une industrialisation de la production du papier -, n'est pas employée dans le système d'impression orientale traditionnelle. Et, si l'histoire du papier est relatée dans les textes chinois depuis longtemps, il en va tout autrement pour ce qui regarde les pratiques et les métiers de l'impression : ce n'est en effet qu'au siècle dernier qu'un traité sur ces techniques a été rédigé.

Malgré cela, l'association de l'histoire des livres et des imprimés à l'histoire du papier reste habituelle, même en dehors des ouvrages à vocation encyclopédique, qu'il s'agisse d'études sur la consommation ${ }^{5}$ ou sur la culture locale ${ }^{6}$. Bien sûr, cette approche n'est

3. Alors que je concluais cet article, j'ai lu l'étude de T.H. Barrett, « The Women Who Invented Notepaper: Towards a Comparative Historiography of Paper and Print ", Journal of the Royal Asiatic Society, series 3, 21, 2 (2011), p. 199-210. Barrett est aussi sensible à la façon dont les différentes traditions historiques ont parlé du papier. Cependant, il met en avant un autre aspect de la question, à savoir le fait que les auteurs occidentaux ont peu traité la question du papier dans leurs histoires du livre du vieux continent (p. 200-201). Dans la mesure où le papier est une invention indiscutablement orientale, à la différence de l’imprimerie, il considère que cette approche " silencieuse » du thème du papier est une manifestation de l'eurocentrisme qui domine la discipline. Tout en comprenant la logique de son discours, je maintiens l'idée qu'une association " papier et imprimerie », si elle est pertinente pour l'Europe, est insuffisante pour rendre compte du cas chinois. D'ailleurs, dans la suite de l'article, Barrett le montre très bien, car comme il l'écrit, « [...] clearly paper had a cultural value in China as part of the technology of the creative scholar that it did not have in Europe, perhaps in part due to the importance - which continues to this day - of calligraphy as a means of self-expression in the public arenli. » (p. 209-210).

4. Au-delà de ce qu'on peut penser des idées de Wolfgang von Stromer (1922-1999), ce n'est pas un hasard s'il a associé l'arrivée de l'imprimerie d'Orient aux activités d'une famille de papetiers ayant installé les premiers moulins en Europe de Nord : voir son ouvrage, Le mystère Gutenberg de Tourfan à Karlstein. Les origines chinoises de l'imprimerie, Genève, Slatkine, 2000, surtout p. 52-53. Les chapitres sur le papier dans The Invention of Printing in China and Its Spread Westward de Thomas Francis Carter (1882-1925) ont justement la même fonction : expliquer que, de même que le papier a été introduit d'Asie, de même ce processus a pu se produire pour l'imprimerie, quoique Carter se garde de le dire explicitement, faute de preuves directes.

5. C'est le cas de l'ouvrage de Wei Huaxian, qui consacre un chapitre au papier et, plus particulièrement, les pages 155-159 au thème de l'utilisation du papier pour les livres imprimés ; Wei Huaxian 魏華仙, Songdai silei wupin de shengchan he xiaofei yanjiu 宋代四類物品的生產和消費研究, Chengdu, Sichuan chuban tuanji - Sichuan kexue jishu chubanshe, 2006. Ce livre traite, au niveau national, de certaines problématiques qui ont été approfondies dans cet article au niveau régional. L'approche de Wei veut surtout mettre en avant la consommation du papier, de la part des particuliers mais aussi des institutions publiques ; pour ce faire, certaines interprétations de sources sont forcées, mais les matériaux proposés au lecteur restent intéressants.

6. Par exemple dans le Sud du Hunan, voir Feng Qicai 冯瀷才 (éd.), Zhongguo muban nianhua jicheng : Tandou juan 中國木版年畫集成 滩头卷, Beijing, Zhonghua shuju, 2007,p. 264-267. 
pas toujours dénuée de signification, par exemple dans le cas des producteurs d'estampes qui furent jadis papetiers ${ }^{7}$. Je dirais même que le moment serait venu pour que l'association devienne pleinement significative, puisque les institutions chinoises recensent actuellement toutes leurs collections, en y incluant des fonds locaux ${ }^{8}$, jusqu'à présent marginalisés. Elles devraient pouvoir établir un échantillonnage des papiers localement disponibles et, procédant pour des localités d'abord proches, ensuite plus éloignées, avoir ainsi une idée des circuits des papiers effectivement utilisés pour l'imprimerie. Avec une telle analyse, on pourrait pour une fois parcourir le chemin inverse de celui trop souvent emprunté qui consiste à définir le papier selon des catégories célébrées dans les textes et des caractéristiques souvent floues ; on comprendra bien ce problème à la lecture des pages suivantes. Il est difficile de savoir si des travaux de la sorte sont en cours, et si de nouvelles informations seront bientôt disponibles ${ }^{9}$. En attendant que les techniques de laboratoire bouleversent les pratiques de l'historien, j'ai donc repris l'étude du papier du sud de l'Anhui, en faisant des choix, notamment à propos des sources analysées.

\section{Sources et problématiques}

Dans cette étude, je vais présenter essentiellement des extraits de monographies locales de l'Anhui, en commençant par la plus ancienne et la plus célèbre d'entre elles le Xin'an $z h i$ 新安志 $\mathrm{du} \mathrm{XII}{ }^{\mathrm{e}}$ siècle, correspondant au territoire de la préfecture -, pour arriver aux plus récentes, comme le Huizhou fuzhi 徽州府志 du XvI ${ }^{\mathrm{e}}$ siècle. Il sera aussi question de monographies de circonscriptions administratives plus restreintes, comme le district, pour la dynastie des Qing (1644-1911). D'ailleurs, il est intéressant de s'arrêter sur le rapport entre ces textes - informatifs à propos du papier - et les catégories d'ouvrages qui les contiennent, autrement dit sur la façon dont les « formats » des œuvres ont imposé diverses formulations à des contenus similaires. Même si les monographies forment un genre assez codifié et que les responsables de ces compilations sont toujours des administrateurs, on y constate des évolutions, qui sont dues à un changement dans l'organisation et le développement des contenus au fil du temps, mais aussi peut-être aux compilateurs, plus ou moins compétents, travaillant en solo, en petits groupes ou collectivement. De plus, les ouvrages révèlent un rapport plus ou moins étroit entre administrations centrale et locale

7. Imprimerie et papier sont associés dans le chapitre 9 de Hu Huakai 胡化凱 \& Zhang Binglun 張秉倫, Huizhou keji 徽州科技, 《Huizhou wenhua quanshu 》徽州文化全書, Hefei, Anhui renmin daxue chubanshe, 2005. Voir aussi l'ouvrage de Zhang Binglun (1938-2006), co-signé par Fan Jialu 樊嘉祿 \& Fang Xiaoyang 方曉陽, Zaozhi yu yinshua 造紙與印刷, “Zhongguo chuantong gongyi quanji 》 中國傳統工藝全集, Zhengzhou, Daxiang chubanshe, 2005.

8. En travaillant sur les généalogies de Huizhou conservées à la Bibliothèque nationale de Chine qui datent essentiellement de la dynastie des Ming (1368-1644), j'ai souvent pensé qu'il serait important de mener une étude technique sur les fibres du papier de ces ouvrages qui, par nature, sont « locales ». Les dates, mais aussi les lieux de réalisation y sont toujours indiqués avec précision, parfois un village ou un hameau. Établir un échantillonnage des papiers utilisés pour les généalogies aurait été utile pour analyser d'autres livres, y compris ceux destinés au marché. Cela serait une façon de pallier le manque d'information dans les sources écrites sur les livres illustrés de la même époque : ces publications sont des objets de luxe produits par des ateliers privés et les archives privées des commerçants et des artisans de la Chine impériale font cruellement défaut. Sur ces généalogies de Huizhou, voir le catalogage informatique en libre accès sur le site de l'EFEO, entièrement réalisé à partir des originaux : $M$. Bussotti et Bao Guoqjang 鮑國強, http://www.efeo.fr/hase_huizhou/.

9. Un grand programme national de recension d'ouvrages, lancé en Chine continentale en 2007, porte aussi sur des projets de restaurations prioritaires, ce qui implique logiquement la collecte d'informations sur le papier. 
(cette dernière étant l' " éditeur » des monographies locales), ce qui traduit une politique centralisée à des degrés différents. Parfois, les informations transitent à l'identique des mentions dans les monographies à celles des recueils encyclopédiques. Ces contenus finissent ensuite par être transcrits dans des sources historiques plus générales. Beaucoup plus tard, on les retrouve dans les histoires du " papier et imprimé » qui ont façonné, au $\mathrm{xx}^{\mathrm{e}}$ siècle, notre connaissance et notre représentation de la « civilisation chinoise $» 10$.

À ces documents ont été associées d'autres sources contemporaines, qui confirment les liens entre des publications de nature différente et les " emprunts " d'information que les compilations/les compilateurs opèrent, en copiant les un(e)s sur les autres. En revanche, j'ai cherché à m'affranchir le plus possible des textes - notes, poèmes, traités d'amateurs - qui ont une approche artistique et esthétique du sujet. Conçus et théoriquement diffusés dans des circuits " privés ", composés par des lettrés qui ont montré souvent par leur silence leur désintérêt pour certaines techniques (dont celles de l'impression), ils indiquent si, et jusqu'à quel point, le procédé ou l'objet manufacturé dont il est question a été " promu » aux yeux des élites, intégré dans un patrimoine culturel qui mérite la consécration par l'écriture. Mais ces textes ont aussi, avec le temps, une forte tendance à la citation et à la répétition. Et même si les figures du lettré et du fonctionnaire se superposent, surtout pour la période la plus ancienne traitée dans cet article, le papier décrit dans les monographies locales n'est pas uniquement voué à être un « trésor du lettré ». C'est tout d'abord un produit du monde agricole, soumis de ce fait à l'impôt. Le papier est donc un produit économiquement important, dans un milieu rural où sa production s'associe aux travaux des champs ou à d'autres exploitations. Mais il s'inscrit aussi dans des circuits plus larges qui, par les prélèvements fiscaux. le commerce, les échanges et les cadeaux, l'amènent des lieux de production aux villes, jusqu'à la capitale. Il est également un produit manufacturé décrit dans quelques textes, certes brefs, mais qui se démarquent par leur technicité et leur précision.

Ayant ainsi fait le deuil de certaines sources " et de certaines problématiques, prenant du recul par rapport à mes premiers travaux inspirés de la relation entre papier et imprimerie, ce qui me semblait ressortir le mieux dans le cas du papier dans l'extrême sud de l'Anhui (Carte 1) était une concentration d'information assez riche dans un territoire relativement restreint. Sauf à écrire une histoire littéraire et générale du papier en Chine, rester concentré sur cette zone géographique trahit évidemment l'ambition de présenter ce cas comme emblématique pour l'ensemble du pays, tout en gardant ses limites présentes à l'esprit : d'autres aires géographiques auraient pu être choisies ${ }^{12}$; cet empire, trop souvent décrit en raison de son unicité, a connu des divisions et des phases

10. L’importance des monographies locales est aussi signalée dans le volume sur l'agro-industrie de l'encyclopédie de Joseph Needham. Tout en mettant en garde les lecteurs contre les « adaptations » que les auteurs peuvent avoir introduites dans la narration, les monographies sont signalées comme des sources riches et relativement fiables, tandis que le papier est présenté comme l'un des produits dérivés des récoltes agro-industrielles à étudier, même si son cas n'est pas développé dans l'ouvrage en question. Voir Christian Daniels, Nicholas Kay Menzies, Agro-industries: Sugarcane technology, Forestry, dans Joseph Needham (éd.), « Science \& civilisation in China », vol. 6, part 3, Cambridge, Cambridge University press, 1996 : « Local gazeteers as sources for agro-industries », p. 45-51.

11. Bien évidemment, mon choix ne signifie pas que d’autres sujets ne méritent pas d’être abordés : une anthologie de textes chinois sur le papier traduits en français est annoncéc par Jean-Pierre Drège. Je remercie ce dernier de mavoir signalé les ouvrages de F. A. Bischoffet de Hans Ulrich Vogel, cités plus bas dans cet article.

12. C'est notamment le cas du Sichuan qui, pour les premiers siècles du deuxième millénairc, est très important, et de toute la zone du Jiangxi-Zhejiang-Fujian pendant les dynasties Ming et Qing. 
où le contrôle central s'est exercé différemment (nous le verrons tout spécialement à propos de la production du papier). De plus, bien que le papier d'écorce soit privilégié dans les pages qui suivent, il ne faudrait pas oublier qu'ont existé ou que persistent des productions importantes de papiers faits à partir d'autres matériaux comme le chanvre, le rotin ${ }^{13}$ ou le bambou.

La zone géographique choisie s'inscrit également en contrepoint de la longue période traitée, pour laquelle le lieu circonscrit permet de tracer une ligne directrice. Le propos est donc organisé chronologiquement en trois parties, abordant tout d'abord la dynastie des Song (960-1279), puis la période Yuan-Ming $\left(\mathrm{XIII}^{\mathrm{e}}-\mathrm{XVII}^{\mathfrak{c}} \mathrm{s}\right.$.) et enfin la dernière dynastie ainsi que la période républicaine (du milieu du $\mathrm{XVII}^{\mathrm{e}} \mathrm{s}$. au milieu du $\mathrm{XX}^{\mathrm{e}} \mathrm{s}$.). Ces longues durées sont parsemées d'informations et de données qui méritaient d'être relevées, mais qui n'étaient malheureusement pas suffisamment resserrées et explicites pour fournir en elles-mêmes un récit vraiment continu. Sans avoir la prétention d'utiliser des références célèbres à propos des « temps longs » de l'histoire, cette approche est également une façon de s'adapter aux sources disponibles ou choisies ${ }^{14}$. Le récit des événements successifs permet de comprendre des évolutions, notamment celle du contrôle par l'autorité centrale qui semble s'affaiblir pendant les dernières dynasties, du moins par rapport au papier. Au contraire, l'intérêt envers ce produit semble être particulièrement vif sous la dynastie des Song. L'Empire chinois organise alors véritablement son administration, fondée sur un système de recrutement massif par concours : lettrés et fonctionnaires, diplômés ou aspirants, sont des « intellectuels » professionnalisés qui consomment du papier dans leur travail et leur fonction, mais aussi dans leur vie quotidienne. C'est bien à ce moment-là que l'impression xylographique se développe et que la jonction entre papier et imprimé, dont nous parlions en ouverture de cette introduction, se fait, sans que la fonction et les utilités du papier se limitent à cette unique application. Dans les sources écrites de l'administration locale et centrale ici prises en compte, lorsque l'impression est évoquée, il n'est pas question de livres, mais de papier-monnaie.

13. On peut comprendre l'importance de ces deux plantes au vu de leur présence dans les anciens exemplaires imprimés encore conservés, mais aussi par la lecture des sources. Par exemple, dans la

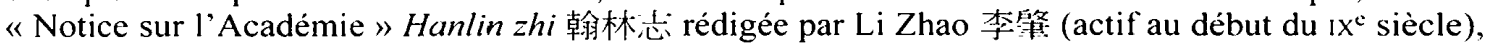
on trouve une énumération de papiers en chanvre ou en rotin spécifiquement utilisés pour différentes sortes d'écrits et codifiés sur la base des couleurs et des motifs ; voir F. A. Bischoff, La forêt des pinceaux. Étude sur l'Académie du Han-lin sous la dynastie des T'ang et traduction du Han lin tche, "Bibliothèque de l'Institut des hautes études chinoises », vol. XVII, Paris, PUF, 1963, p. 49, 50-54.

14. À noter que le récent ouvrage collectif de Dagmar Schäfer (éd.), Cultures of Knowiedge: Technology in Chinese History, Leiden, Brill, 2012, revendique une approche sur une longue période, afin d'être à l'unisson avec les travaux d'histoire globale (p. 4). Mais là aussi, il me semble que cette perspective sur la durée est dictée par les sources : on le voit dans l'article de Schäfer sur le textile et dans celui de Francesca Bray sur les traités agricoles qui couvrent des siècles sinon des millénaires. En revanche, Luo Wenhua 維文化, qui utilise des documents d'archives, ne s'intéresse qu'à une mission d'artisans népalais à la Cour vers 1744-1745 (« Sympathetic Relations: Foreign Craftsmen at the Qing Court », dans op. cit., p.127-146). 


\section{La dynastie des Song (960-1279) : la monographie locale Xin'an $z h i^{15}$}

La pertinence des textes relatifs au papier dans la " Monographie locale de Xin'an » du XII ${ }^{\mathrm{e}}$ siècle confirme la célébrité dont jouit cet ouvrage. Deux aspects notamment y sont développés, dont celui de " produit du lettré », d'une façon très concrète et éclairante à propos de quelques papiers locaux typiques pour leur taille et leur épaisseur. Mais tout d'abord il y est question du papier offert en tribut ou recueilli avec un engouement méthodique dans les provinces par une administration efficace : celui-ci était nécessaire pour alimenter la cour, une pléthore de bureaux et de fonctionnaires, ainsi qu'un nouveau système monétaire qui se mettait en place. Même si le raisonnement peut paraître des plus banals, qu'arriverait-il de nos jours si, malgré la généralisation de l'usage de l'informatique, le papier venait à manquer à la Fonction publique ou au Trésor ? Nous allons lire comment ce produit a pu être au cœur du système bureaucratique qui se déploie sous les Song. On peut ainsi saisir l'importance d'un lieu, le sud de l'Anhui, qui en était producteur, en quantité et en qualité, et qui pendant la période des Song du Sud n'était pas trop éloigné de la capitale. Voici donc qu'un discours sur le papier revendiquant l'importance du produit pour des fonctions autres que celles les plus souvent évoquées - les supports de peintures et de calligraphies, les feuilles de livres manuscrits et imprimés - trouve des témoignages dans les pages des monographies, écrites par et pour l'administration centrale et provinciale. On constate alors la valeur d'une autre « littérature » que les biji (筆記 《notes au fil du pinceau ») ou les poèmes; tout en contribuant à la constitution d'une même mémoire savante et écrite, elle met en avant d'autres formes matérielles ou plutôt d'autres récits à propos d'une même forme matérielle, car certains des papiers réservés à la Cour et à ses bureaux étaient vraisemblablement aussi précieux que ceux pour une peinture ou une calligraphie. Prendre en compte les données, un peu « sèches » et parfois contraditoires, des énumérations administratives permet de faire ressortir des informations utiles et nouvelles en dehors des appréciations esthétiques des lettrés et des amateurs, mais n'oublions pas pour autant que tous ces écrits émanent de la même élite intellectuelle. Les gens du commun, les (agriculteurs)-artisans et les commerçants qui sont à la base des circuits où le papier est produit, échangé, commercialisé, restent complc̀tement « muets». C'est pourquoi nous avons décidé de terminer cette section sur une anecdote qui, bien que relatée également par un fonctionnaire-lettré, nous décrit un monde où l'économie du petit commerce, la religion, les croyances locales s'entremêlent, permettant de ne pas oublier complétement ces aspects concernant les utilisations les plus communes du papier.

15. Là où elles sont disponibles, les sources en ligne ont été utilisées, consultées entre novembre 2013 et décembre 2014. Ce sont essentiellement la version en ligne du Xin'an zhi et du Songshi 尔史 inclus dans le Siku quanshu (http://10.34.2.9.ezp-prod 1. hul.harvard.edu/scripts/skinet.dll?OnTrigger?code=1124), abrégés comme suit : XAZ-SKQS et SS-SKQS. La version en ligne du Song huiyao jikao 宋會要輯稿 a été consultée à la même période : http://songhuiyao.hul.harvard.edu.ezp-prod I hul.harvard.edu/; il aurait d'ailleurs été bien plus difficile d'effectuer autrement ces recherches ciblées : tout en restant incomplet et imparfait, cet excercice voudrait au moins montrer la richesse de la matière qui reste à exploiter. Le titre Song huiyao est abrégé en SHY; il est suivi des indications pour retrouver les informations dans une édition imprimée (Beijing, Zhonghua shuju, 1957). Le Xu Zizhi tongjian changbian 續資治通鑑 長編 de Li Tao 李率 (1115-1184), abrégé en XLC'B, est disponible en ligne à l'adresse : http://www. guoxue 123.com/shibu/0101/01 jzztjcp/037.htm; nous avons également consulté l'édition faite en 1979 à Pékin par la Zhonghua shuju, dont le numéro du volume et la pagination sont donnés entre parenthèses. 


\section{Le Xin'an zhi et ses contenus}

Le Xin'an zhi de Luo Yuan 羅願 (1136-1184, zi Ruiliang 瑞良, hao Cunzhai 存齋, jinshi en 1166) date de 1175 (deuxième année de l'ère Chunxi 淳熙); c'est la première monographie consacrée à Xin'an, ancien nom de la préfecture de Huizhou (Carte 3). Il n'est pas impossible que, comme bien des monographies locales, elle soit une cuvre collective, mais elle nous est parvenue comme ayant été écrite par Luo, qui était originaire des lieux, et préfacée par le préfet Zhao Buhui 趙不悔. Le Xin'an zhi est célèbre, car il s'agit de l'une des plus anciennes monographies locales chinoises dont le contenu ait été conservé. Les éditions connues datent en réalité toutes de la dynastie des Qing ${ }^{16}$, mais développer ce point nous amènerait loin de notre sujet sans apporter grand chose à notre présentation à propos du papier, du moment que l'on considère que le texte actuel est fidèle à l'original.

Luo Yuan était le descendant à la huitième génération d'une famille réputée être arrivée à Chengkan 呈坎, une localité à $40 l i$ au nord-ouest de Shexian, lors des désordres qui ont précédé l'établissement de la dynastie des Song. Engagé dans l'administration publique, il a laissé deux autres titres d'ouvrage : le Erya yi 爾雅翼 (Aides à la lecture du Erya) et le Ezhou xiaoji 鄂州小集 (Petite collection d'Ezhou), Ezhou étant un de ses surnoms, dû à ses années de service dans cette localité correspondant aujourd'hui à Wuchang 武昌, dans l'actuelle province du Hubei.

Le texte de la monographie de Xin'an frappe le lecteur par l'organisation de son contenu, déjà très structuré malgré sa datation ancienne ; en revanche, on y trouve ni carte ni présentation tabulaire des données. Nous ne pouvons pas exclure que cette présentation linéaire des informations dépende de manipulations effectuées sur la mise en page originale et désormais oubliée, qui seraient donc intervenues après coup, par exemple au moment de la réalisation des versions aujourd'hui conservées. Les contenus des chapitres peuvent être ainsi résumés :

- le premier chapitre, consacré à la préfecture (zhoujun 州郡), inclut les entrées 《 classiques », comme les changements des divisions administratives (vange 沿革) et leurs correspondances astronomiques (fenye 分野), les coutumes locales (fengsu 風俗) et les (anciens) fiefs (fengjian 封建). Viennent ensuite la description des structures et infrastructures présentes sur le territoire - les principaux bâtiments de l'administration, les routes, les écoles, les temples... - mais aussi les données d'enregistrement (hukou 戶口) ou les noms de lignages (xingshi 姓氏).

- le deuxième chapitre est divisé en deux sections complémentaires sur les produits locaux et les différentes formes d'imposition, sujet sur lequel nous allons revenir.

- les trois chapitres suivants sont consacrés aux districts de She 歌 (chapitre 3), de Xiuning 休寧 et Qimen 祁門 (chapitre 4), de Wuyuan 丞源, Jixi 績溪 et Yi 黟 (chapitre 5). Dans cette partie, les contenus déjà exposés au niveau de la préfecture sont systématiquement repris : les changements successifs de l'administration de ces territoires, la population et les impôts, le territoire physique et les voies de communication, ainsi que les temples, les lieux connus, les inscriptions, etc.

16. Xiao Jianxin 蕭建新, 《Lun Xin'an zhi keben de xitong he jiazhi 》 論《新安志》刻本的系統和 價值, Huazhong keji daxue xuebao (shekeban), 3 (2006) et Zhang Yingpin 張英聘, Mingdai Nanzhili fangzhi yanjiu 明代南直隸方志研究, Beijing, Shehui kexue wenxuan chubanshe, 2005, p. 54-55. En plus du XAZ-SKQS, voir le fac-similé Chunxin Xin'an zhi 淳熙新安志 (Monographie de Xin'an de l'ère Chunxi), 1888, réédition reproduite dans «Zhongguo fangzhi congshu»中國方志橗畫 (Recueil des monographies locales de Chine), Taipei, Chengwen chubanshe, 1970, abrégée en XAZ-F ou la récente édition critique de Xiao Jianxin, “Xin'an zhi » zhengli yu yanjiu 《新安志》整理與研究, Hefei, Huangshan shushe, 2008, abrégée en XAZ-EC. 
- les chapitres 6 à 9 sont composés de biographies, d'abord des personnages classés comme 《 ancêtres méritants » dans la voie de la sagesse (xianda 先達), des hommes qui ont vécu entre le $\mathrm{VI}^{\mathrm{e}}$ siècle et le $\mathrm{XII}^{\mathrm{e}}$ siècle, dont Xianjun shangshu 先君尚書 (alias Luo Ruji 羅汝揖, le père de Luo Yuan). Viennent ensuite les noms des licenciés aux concours et des officiers de l'administration civile et militaire, les « personnes loyales » (yimin 義民) parmi lesquelles quelques femmes; ensuite les «taoïstes » et les «bouddhistes » (xianshi 仙釋). Enfin, est presentée la liste des préfets (mushou 牧守) qui ont exercé sur place, à partir de l'époque du royaume de Wu (222-258) jusqu'aux années 1160.

- le chapitre 10 (《 notes diverses » zalu 䌖録) traite des affaires humaines (renshi 人事), de la poésie (shihua 詩話) et des arts (zayi 雜藝), ainsi que des « trésors du lettré », tels les pierres à encre yan 硯, le papier $z h i$ 紙 et l'encre mo 墨. L'ouvrage se conclut sur les «prédictions » (dingshu 定數), les « esprits et faits prodigieux » (shenyi 神異), et les « choses entendues 》 (jiwen 記聞).

En fait, même si les titres de chapitres sont formulés différemment dans d'autres monographies au cours des siècles et si l'ordre de présentation des données est parfois modifié, on trouve déjà dans le Xin'an zhi les thèmes proposés aux lecteurs à l'époque impériale tardive, moment où les monographies locales atteignent leur développement maximal ${ }^{17}$. Les trois chapitres consacrés à Shexian, Xiuning, Qimen, Yixian, Jixi et Wuyuan traitent de districts existant encore de nos jours, mais Wuyuan est inclus dans la province du Jiangxi depuis le début du $\mathrm{Xx}^{\mathrm{e}}$ siècle (Carte 2$)^{18}$. Avant de clore par un chapitre de « Notes diverses » où l'on retrouve aussi des informations sur le papier, les chapitres 6 à 9 du Xin'an zhi des Song citent un grand nombre de personnalités, avec des notices plus ou moins élaborées. Ces énumérations préludent à une autre caractéristique des monographies qui se développe sous les dernières dynasties, notamment dans notre localité : l'hypertrophie des sections biographiques, y compris des chapitres entièrement consacrés aux biographies de femmes.

\section{Les produits du territoire comme tributs : l'exemple du papier}

Le papier de Xin'an est un produit local soumis à taxation, ainsi qu' un des « trésors du lettré "; il est donc traité à deux reprises dans la compilation de Luo Yuan. Le chapitre 2 du Xin'an zhi est divisé entre les «produits locaux » (wuchan 物産) et les «tributs et impôts " (gongfu 貢賦). Parmi les produits, sont énumérés les céréales et différents végétaux, les patrimoines animaliers et autres « richesses» (huohui 貨賄). Dans cette section, le papier revient à deux reprises, tout d'abord parmi les « arbres et leurs fruits " (muguo 木果), avec le thé et la laque, à la suite d'une liste de noms d'arbres. Mais, localement, le papier sert aussi à protéger : imbibé d'huile de $s h i$ 柿 (Diospyros [kaki]), il permet

17. T. Brook, The Chinese State in Ming Society, London, Routledge Curzon, 2005, p. 43-44; voir aussi Pierre-Étienne Will, «Chinese Local Gazetteers: An Historical and Practical Introduction », Notes de recherches du centre Chine, $\mathrm{n}^{\circ} 3,1992$, surtout p. 9-14.

18. La « capitale » de la préfecture était située le plus souvent à Shexian, parfois à Xiuning, tandis que de nos jours, le chef-lieu administratif se trouve à Tunxi 屯溪. Cette municipalité était un village à la confluence des cours d'eau alimentant le Xin'an jiang 新安汀. et appartenait par le passé au territoire du district de Xiuning. Elle est le chef-lieu de la région administrative de Huizhou, qui a perdu Wuyuan, mais a acquis des territoires au nord de l'ancienne préfecture. De l'ouest à l'est, ces nouveaux territoires sont le district de Shitai 不治, la municipalité de Huangshan 革り」柿 et le district de Jingde 旌德 (Carte 1) ; cf. Huizhou diqu jianzhi 徽州地简志, s.1., Huangshan shushe, 1989, p. 50-53. 
d'envelopper ${ }^{19}$ les branches des arbres fruitiers; on l'utilise plus particulièrement pour protéger les fruits d'une sorte de poirier (gantang 甘棠, Pyrus betulaefolia Bunge) qu'on cueillait après les premières gelées, avant qu'ils ne soient endommagés par les attaques des insectes, susceptibles de nuire à leur commercialisation. Ce produit est loin du « trésor du lettré » et le texte décrit une utilisation du papier par des paysans. Nous pouvons en déduire qu'ils disposaient d'un papier suffisamment épais et résistant aux intempéries pour être efficace. Notons que l'utilisation d'huiles végétales destinées à consolider le papier pour pouvoir l'employer dans différents secteurs, dont la construction, est attestée par d'autres sources des Song ${ }^{20}$.

Plus loin dans la monographie, après qu'il est expliqué que She n'est plus producteur d'or et d'argent ${ }^{21}$, il est question du bois précieux, du thé, de la laque (l'arbre à laque), mais aussi de certains produits manufacturés, tels l'encre, les nattes de bambous (dian 䉪) et, plus particulièrement, le papier. Nous apprenons que sa production se fait à partir du « mûrier à papier » (chu 楮, broussonetia papyrifera) :

Parmi les papiers il y a ceux qui sont appelés maiguang 笠光 (éclat de blé), baihua 白滑 (blanc poli), bingyi 冰翼 (aile de glace) et ningshuang 凝霜 (gelée). De nos jours, entre les districts de She et de Ji, comme il y a un lieu qui s'appelle Longxu 龍琵, le papier qui y est produit est nommé papier Longxu (littéralcment « Barbe du dragon »22).

19. Je n'ai pas trouvé jusqu'à présent d'autres sources aussi anciennes confirmant cette pratique, mais elle n'est pas étonnante pour autant. D'ailleurs le terme $s h i$ 柿 indique un genre comprenant des centaines de plantes, dont l'arbre que nous connaissons pour ses fruits, ainsi que d'autres arbres qui produisent des vernis et des huiles utilisés pour l'imperméabilisation ; cf. Francine Fèvre et Georges Métailié (Dictionnaire Ricci des plantes de Chine, Paris, éditions du Cerf, 2005, p. 408 et 553). Il faut également signaler que selon ce dictionnaire (op. cit., p. 111), gantang est un synonyme de duli 杜梨尘, et que celui-ci n'aurait pas eu d'intérêt à la consommation alimentaire, ce qui semble contredire notre citation ; en revanche, il aurait été utilisé comme excipient.

20. Wei Huaxian (2006, p. 170) signale l'huile d'aleurite (ou d'abrasin tongyou 桐油), et aussi le biyou 碧油, mais cette expression reste plus difficile à interpréter car elle se réfère à une huile de couleur bleuâtre, et non à une substance précise.

21. Selon le Guochao hui yao 㽣朝會要, des circonscriptions produisent ces métaux, mais ce n'est pas le cas de She. En fait, l'ère Chunxi (1174-1189) marque l'apogée d'une augmentation d'impôts qui dure quelques décennies; quelques années après la compilation de la monographie, une politique de limitation d'impôts pour alléger leur poids sur la population est mise en place. Cf. Christian Lamouroux, Fiscalité, comptes publics et politiques financières dans la Chine des Song, Paris, Collège de France, IHEC, 2003, «L'accroissement des charges fiscales au XII" siècle », p. 212-214; la note 212 cite l'exemption des charges fiscales en or et en argent.

22. Dans la reprise de ce passage par des ouvrages plus tardifs, aux deux caractères Longxu est associé shan U; il s'agit ainsi de la « montagne de la barbe du dragon ». La montagne en question se trouve précisement aux confins de deux districts, dans la partie la plus orientale (voir la Carte 2), et, selon les sources contemporaines, elle dépasse de peu les 1000 mètres. Le papier de Long Xu sera aussi cité par Tu Long 屠隆 (1543-1605) : “Sous les Song, il y avait du papier de She, de nos jours Shexian à Huizhou : dans un lieu appelé Longxu on produisait un papier d'un blanc éclatant très agréable [...] 》 Cf. Zhimo biyan jian 紙墨筆硯笺, dans « Meishu congshu » (1936, p. 1v), reproduit à Yangzhou, Jiangsu guji chubanshe, 1986, vol. 2, p. 1219. À propos du terme longxu, une ambiguïté demeure, car ces caractères sont communs à beaucoup de noms de plantes utilisées comme excipients ; par exemple, le Potamogeton pectinatus, qui vit en eaux douces, en hauteur. Sur ces termes, voir Fèvre \& Métaillé, 2005, p. 84 et 412. De plus, selon Lucien Polastron, au Sichuan, une plante portant ce nom (longxucao 龍㰮荤, qu il traduit comme Juncus setchuensis) est employée de nos jours pour produire, associée à du bambou et à du mûrier, un papier dit " de xuan ». Cela est d'autant plus frappant que le terme xuan désigne aussi la localité du sud de l'Anhui qui produit du papier de qualité, sur lequel on reviendra en fin d'article. Cf. L.C. Polastron, Le papier: 2000 ans d'histoire et de savoir-faire, Paris, éditions de 1'Imprimerie nationale, 1999, p. 39. 
Généralement, les eaux à Xin'an ${ }^{23}$ sont si pures que l'on peut voir le fond et on en profite pour y faire longtemps macérer du mûrier à papier. [En faisant le papier, on l'agite légèrement; il ressemble] au blanc de jade : c'est l'eau qui fait sa couleur. En fin d'année, on casse la glace pour en faire, ce qui en augmentant sa résistance fait sa valeur.

Un autre texte des Song va reprendre la conclusion de cette première citation : le Shanlu 剡錄 (Notes sur Shan, alias le district de Shen 嵊縣 dans le .Nord-Est de la province du Zhejiang, voir Carte 3) de Gao Sisun 高似孫 (1160-1220), avec une préface de 1215. Le passage revient dans une énumération de papiers célèbres, qui est connue en tant que telle : on a ainsi tendance à oublier qu'il s'agit ici d'une section du septième chapitre d'une monographie et qu'une fois encore, l'information à propos du papier nous parvient grâce à cette catégorie d'ouvrages. Le Xin'an zhi est cité dans le Shanlu à deux reprises ${ }^{24}$ pour présenter deux types de papier, dont le « papier de glace pilée » (qiaobing $z h i$ 敲冰紙). Cette expression qui, dans le Xin'an zhi, désignait la méthode pour obtenir un papier très résistant, sert dans le Shanlu à nommer le même produit. Seulement une quarantaine d'années se sont écoulées, et le nouveau texte s'est approprié l'autre, en modifiant un peu le discours. En plus des citations poétiques et de celle reprise du Xin'an $z h i$, Gao dit que l'expression était utilisée à Yue 越 (Nord du Zhejiang, région à laquelle Shan appartient) et ajoute qu'à Shan ce papier est excellent ${ }^{25}$, car les eaux occidentales sont très pures. Dans la montagne, on trouve beaucoup de plantes rampantes et du mûrier à papier (teng chu 藤楮 ${ }^{26}$ ), et on considère qu'un meilleur résultat est obtenu quand on pile de la glace, des qualités étant associées à l'eau hivernale. De nos jours aussi, l'eau froide reste indispensable à la production artisanale du papier asiatique, car la chaleur est connue pour nuire au liant de la pâte ${ }^{27}$.

Au XIII siècle, le passage du Xin'an zhi ci-dessus sera repris presqu'à l'identique dans la compilation géographique Fangyu shenglan 方輿勝覽 ${ }^{28}$. Dans cette synthèse sur le territoire des Song du Sud, il est aussi noté que les montagnes produisaient du bois

23. C'est-à-dire à Xin'an, à moins qu'il ne s'agisse ici des eaux du Xin'an, le fleuve qui portait le même nom, et qui coule vers l'est.

24. L'autre citation concerne le fameux papier du Chengxin tang 澄心堂, sur lequel nous allons revenir ci-après.

25. Plus tard, d'autres auteurs ont éliminé la référence à la monographie de Xin'an : ce papier est alors présenté comme un produit typique du Zhejiang septentrional. C'est le cas de Yang Shen 楊慎 (1488-1559), qui en parle dans son Taishi Sheng'an wenji 太史仆庵文集 en 66 juan : cette citation est mentionnée dans le Hanyu dacidian (vol. 5, p. 500), qui estime que le qiaobing zhi est un produit du Zhejiang.

26. Nous avons cité le papier de rotin à la note 13. Sur ce papier connu depuis les Tang, notamment pour cette localité, voir aussi Pan Jixing, 1998, p. 140-141. En revanche, Camille Schmitt cite le ziteng 紫藤 (glycine, Wisteria sinensis) comme un dispersant dans la pâte à papier; cf. La médecine de l'art. Conservation des calligraphies et peintures chinoises en rouleaux à partir des textes de la dynastie Ming (1368-1644), Paris, Picquier, 2011, p. 261.

27. Voir Érik Orsenna, Sur la route du papier. Petit précis de mondialisation III, Paris, Stock, 2013, p. 103-104, où est décrite la production du papier japonais d'Echizen : les meilleurs produits sont faits en hiver.

28. Voir Zhu Mu 祝穆 (xIII siècle), Fangyu shenglan 方輿勝覽, Taipei, Taiwan Shangwu yinshuguan, 1986, SKQS, ch. 16, p. 177, où les quelques caractères du Xin'an zhi-évidemment déjà peu clairs à l'époque (之成振之似) - ont été éliminés ; dans la traduction de la citation ci-dessus, les mots correspondants sont entre crochets. 
d'œuvre de qualité (shanchu meicai 山出美材) et que, grâce au cours d'eau de Qimen qui se jette dans le Po [yanghu] 鄱陽湖 ${ }^{29}$, les gens envoyaient au Jiangxi le thé, la laque, le papier et le bois, mais gardaient le riz pour leur propre subsistance ${ }^{30}$.

En revenant à la section « Présentation de tributs " (進貢 jingong) dans le chapitre 2 du Xin 'an zhi, trois qualités de ces papiers sont de nouveau citées dans un récit historique, mais cette fois, malheureusement, en dehors des noms, nous n'apprenons rien sur les matériaux utilisés, les méthodes ou les lieux de production :

Selon le Tang Liudian 唐六典 les préfectures offraient en tribut du tissu de ramie blanc au chancelier de la Cour du Palais des revenus (taifuqing 太府卿). Les produits en tissu de chaque région étaient rangés en neuf catégories, des plus beaux aux plus grossiers ; la ramie de She était de septième catégorie. Selon le Tongdian, 15 duan $^{31} \mathrm{de}$ ramie et une natte de bambou furent offerts. Dans le Xintang shu, il est aussi question du papier et du huanglian 黄連 (Coptis chinensis) ${ }^{32}$. Sous notre dynastie, on envoie en tribut du biaozhi 表紙, du papier maiguang, baihua et bingyi, des herbes médicamenteuses séchées, du thé et des tissus fins ${ }^{33}$. Pendant le $5^{\mathrm{c}}$ mois intercalaire de la $4^{\mathrm{e}}$ année jingde (1007), un décret spécial réduisit les produits offerts par soixante-six localités, et sept produits de She en faisaient partie. Et il fut décrété que les fonctionnaires, à l'avenir, ne seraient plus autorisés à lever arbitrairement des surtaxes sous prétexte de contribution tributaire. Pendant l'ère Xining (1068-1077), il y avait 1000 feuilles de (papier) baihua, 10 jin d'encre dalongfeng ${ }^{34}$, et pendant l'ère Yuanfeng (1078-1085),

29. À cette brève citation sur l'envoi de marchandises vers l'ouest, on peut associer ce que Fan Chengda écrit à propos des bois de sapin acheminés par voie fluviale vers le Zhejiang, $\mathrm{cf}$. Fan Chengda 范成大 (1126-1193), Canluan lu 驂鸞錄, “Conghu jicheng jianbian », Taipei, Shangwu yinshuguan, 1966, p. 4. L'ouvrage a été traduit en anglais par James H. Hargett, On the road in Twelfth Century China: The travel diaries of Fan Chengda, Stuttgart, Steiner Verl. Wiesbaden, 1989, p. 185 ; pour une traduction en français de cet extrait, voir ma thèse de doctorat, 1997, p. 23-24.

30. Ce dernier passage à propos du commerce au Jiangxi est extrait d'une autre section de la monographie, au chapitre 1 (p. 5 dans la version du XAZ-SKQS), où, pour la première fois, le papier est cité parmi les produits dont la région faisait commerce - informations que l'auteur du Fangyu shenglan a donc réunies dans son texte. Certains chercheurs interprètent ce passage en concluant que Qimen échangeait le papier et d'autres produits pour obtenir du riz.

31. Le duan 端 est une ancienne mesure de longueur pour les pièces de toile, équivalant à 20-18 尺 chĭ ou pieds selon le dictionnaire Ricci.

32. Le Coptis chinensis est une plantée herbacée ; son rhizome est utilisé comme excipient : voir Fèvre \& Métaillé, 2005, p. 205. Dans l'édition de l'ère Jiajing du Huizhou fuzhi (Monographie de Huizhou), les tributs annuels sous les Tang sont déjà au nombre de six ; cf. le fac-similé inclus dans Beijing tushuguan guji chuban bianji zu 北京圖書館古籍出版编辑組 (éd.), « Beijing tushuguan guji zhenben congkan 》 北京圖書館吉籍珍本趣刊, vol. 29 (Shibu 史部: dililei 地理类), Beijing, Shumu wenxian chubanshe, 1998. Le titre de l'ouvrage est abrégé en HF-JJ.

33. La traduction de ces listes est toujours compliquée : il suffit de comparer cela avec la même citation reprise dans le Huizhou fuzhi de 1502 (ch. 2, p. 51 v-52r), pour constater que le texte est coupé différemment, avec quelques variations de caractères : soit les auteurs disposaient d'une autre version que la nôtre, soit ils ont choisi d'autres formulations par souci de clarté. Dans l'ensemble, le sens reste cependant correct. Le Huizhou fuzhi de l'ère Hongzhi, inclus dans Tianyi ge cang Mingdai fangzhi xuankan 天一閣藏明代方志選刊, Shanghai, Shanghai guji chubanshe, 1964, est désormais abrégé en HF-HZ.

34. La quantité d'encre du " grand dragon et du phénix » était de 100 ding 錠 (onces, lingots) en 1068 selon le SHY, section shihuo 食貨 « ressources en biens et denrées 》, 四一/曆代土貢 (vol. 6, p. 5557 ; ce $142: 41$ ). Elle est ensuite de 1000 plaquettes (qianpian 千片), dans le commentaire du HF-HZ de 1502 , op. cit., p. 52r. Dans ces témoignages, on trouve des adaptations de mesures et de poids, de siècle 
$10 p i$ de ramie blanche ${ }^{35}$, la même quantité de papier qu'à l'époque précédente, mais pas d'encre. Actuellement la ramie est encore à $10 \mathrm{pi}$.

\section{Des réglementations et fiscalités du papier sous les Song}

Des sources très anciennes font effectivement référence à des tributs de papier, à commencer par la Nouvelle histoire des Tang où quatre produits sont cités : la ramie blanche, le store (de bambou), le papier et le Coptis chinensis ${ }^{36}$; seul le papier et la ramie sont cités dans le Songshi ${ }^{37}$. Dans les informations réunies dans le Song huiyao 宋會要 (Recueil des documents institutionnels des Song) ${ }^{38}$, qui viennent confirmer ou éclairer les autres sources, parmi les sept produits tributaires de She, quatre sont des papiers, ce qui est un cas unique dans la liste des lieux bénéficiant d'une réduction des tributs en 1007. Pour les autres localités, selon cette même liste, l'offre est beaucoup plus restreinte en matière de qualité de papier ${ }^{39}$.

Quant au biaozhi 表紙, également présent dans la précédente citation, l'expression sert souvent de nos jours à désigner un " papier de support » pour peintures et calligraphies. Mais, sous les Song, elle désignait un des papiers versés à l'administration, qui aurait pu être utilisé plus anciennement pour des mémoires au trône ${ }^{40}$. Le même Song huiyao nous renseigne sur la distribution régulière des diverses catégories de papier,

en siècle ; ceci suggère que les anciennes unités n'étaient plus significatives pour les compilateurs des périodes suivantes.

35. Un pi 匹, mesure de longueur pour les étoffes, équivalait à 4 zhang $丈$ ou toises, selon le dictionnaire Ricci ; dix pi seraient donc équivalents à une douzaine de mètres : des vérifications seraient nécessaires sur ces unités, mais cela nous éloignerait de notre analyse. Nous ne disposons pas non plus de dimensions pour les « feuilles » de papier dont il est question ici, mais d'autres textes en font mention : voir plus bas, p. 276, 279, 292, 303.

36. Xin Tangshu, ch. 41, p. 1067.

37. Songshi, ch. 88 , p. 2187 . Ces produits sont identiques à ceux décrits pour les années 1078-1085 dans le Yuanfeng jiuyu zhi 元豊豐九域志 (Traité de neuf régions de l'ère Yuanfeng, inclus dans le Siku quanshu et consultés en mars 2014 : http://10.34.2.9.ezp-prod1.hul.harvard.edu/scripts/skinet. dll?OnTrigger?code=18142). Dans cet ouvrage, dont le titre est abrégé en YJZ pour les occurrences suivantes dans cet article, Wang Cun 王存 (1023-1101) et ses collaborateurs donnent peu d'informations à propos des tributs locaux en papier : pour Shezhou sont comptabilisées dix pièces de ramie et mille feuilles de papier (土貢白艼一十正紙一千張); voir aussi ch. 6, vol. 1, p. 242, dans l'édition de la Zhonghua shuju de 1984.

38. À propos de ce précieux compendium compilé sous les Qing par Xu Song 徐松 et de ses multiples informations, cf. Chen Zhichao 陳智超, Jiekai Song huiyao zhimi 解開《宋會要》之謎, Beijing, Shehui kexue wenxian chubanshe, 1995. Voir aussi C. Lamouroux, "A travers le Miroir, Une controverse politique sous les Song », Études chinoises, XXXII-1 (2013), p. 129-151, en particulier p. 133.

39. Il est aussi question du juanzhi 益蜀紙 (qualité de papier blanc et brillant, qui est aussi utilisé pour les certificats d'exemption d'impôt, de service, etc.) dans la région du Sichuan : les lieux cités sont Jianzhou 劍州, Puzhou 酱州, Changzhou 戟州, Suizhou 遂州, Dazhou 達州, Yuzhou 渝州 et Longzhou 龍州, mais cette dernière circonscription pourrait aussi désigner le Guangxi. Ceci ne veut pas dire que ces papiers n'étaient pas produits ailleurs, mais que, localement, la production devait être importante pour modifier les quotas. D'ailleurs, selon Wei Huaxian (2006, p. 127 et 135), ce papier était typique de Wenzhou 溫州 (Zhejiang). On trouve aussi mention d'un changmozhi 長模紙 (papier pour modèles de grand format?) pour la région de Changsha (Danzhou 潭州) au Hunan. Cf. SHY, shihuo君六/金戶 部度支/戶部 (vol. 6, p. 5777 ; ce $148: 9$-10).

40. Dans son traité Wenfang sipu 文房四譜, Su Yijian 蘇易間 (958-996) cite un passage ancien où le terme biao est donné comme un équivalent au «papier blanc » utilisé dans des mémoires adressés à l'héritier présumé, et non au «papier jaune » utilisé pour les mémoires courants - affïrmation qui, d'ailleurs, ne semble pas convaincre Su lui-même ; voir le Wenfang sipu dans la version qu'inclut le "Congshu jicheng » (Shanghai, Shanghai Shangwu yinshuguan, 1936, p. 52, entrée 42). L'utilisation 
dont le biaozhi, aux différents bureaux. Une des premières informations que l'on y trouve - en suivant chronologiquement les données - concerne une nouvelle procédure d'audience, assortie de la création du journal "Chroniques des activités et repos »qui était présenté à l'empereur avant sa transmission au bureau de l'historiographie. Son institution est attribuée à Liang Zhouhan 梁周翰 (929-1009) en 994 ${ }^{41}$. Chargé d'établir des « rapports » à échéances régulières, de cinq ou dix jours selon les autres bureaux de l'administration concernés, le bureau fut doté de deux scribes en écriture régulière, ainsi que d'une disponibilité mensuelle de dix milliers de monnaies et cinq cents feuilles de biaozhi (表紙五百番) ${ }^{42}$. On aurait donc plutôt tendance à voir ces feuilles de biaozhi comme du papier destiné à servir à la rédaction des mémoires au trône et non comme du papier de support, en raison de sa présence dans le lot des " fournitures » attribuées à ce bureau, de la même manière que le sont les deux calligraphes.

D'ailleurs, le caractère pan (que nous avons ici traduit par " feuille ») sert aussi à indiquer des billets de papier-monnaie, alors que, au tout début de la dynastie, l'impression du papier-monnaie ne semble pas encore relever de l'administration centrale ${ }^{43}$. À ce propos, la volonté de rendre uniforme les feuilles de papier pour la monnaie paraît être très tôt une façon de contrôler la première production de papier monnaie, lorsqu'il s'agit encore d'une « affaire " gérée par des maisons privées au Sichuan ${ }^{44}$. Par la suite, l'État en prend peu à peu le contrôle à partir de 1023 (première année de l'ère Tiansheng), sans prévoir au début d'installer ses papeteries; selon Wei Xianhua, ce système se met graduellement en place à partir de 1072, sans que le contrôle en soit jamais tout à fait effectif ${ }^{45}$.

Dans le Song huiyao, pour l'année 1082 (qui clôt une période de réforme de l'administration publique), est cité deux fois un papier jiabiao 夾表紙 ${ }^{46}$, qui ferait donc penser

d'un papier avec des caractéristiques différentes et codifiées pour établir des mémoires au trône, qu'il soit blanc ou plus probablement jaune, ne semble pas incongrue.

41. Cf. XZCB, ch. 35 (vol. 4, p. 779) : d'après le résumé que fait Li Tao, on comprend le fonctionnement de ce bureau : chaque mois, les informations étaient d'abord présentées à l'empereur, avant de redescendre vers le bureau d'historiographie.

42. Ce rythme serait donc différent de celui qui est indiqué dans la note précédente. Cf. SHY, zhiguan 職它 (Fonctionnaires), 二/起居院, vol. 3, p. 2377 (ce 59:11).

43. Voir C. Lamouroux, 2003, p. 215 : celui-ci résume la politique monétaire de la période Song et dresse une liste d'ouvrages de référence. Je m'appuie aussi sur une synthèse inédite du même auteur («L'émergence du papier monétaire dans l'empire chinois $\left(\mathrm{XI}^{\mathrm{c}}-\mathrm{X} 1 \mathrm{I}^{\mathrm{e}}\right.$ siècles) ») qui m'a autorisée à en tirer quelques indications utiles à mon propos.

44. C. Lamouroux rappelle que les sources des Song donnent des dates différentes pour la création du premier papier-monnaie au Sichuan : entre 993 et 1004, ou bien entre 1008-1016, voire précisément en 1011 (cf. note précédente).

45. Wei Xianhua, 2006, p. 159-160: les exemples portent principalement sur le Sichuan qui, selon Wei, reste le centre le plus important pour cette production spécifique pendant toute la dynastie.

46. Il est difficile de trancher sur le sens précis de ces deux caractères; ils apparaissent aussi dans la section «Fonctionnaires " du SHY à propos des Song du Sud ; en 1134 (4 année Shaoxing), il est question de « 100 feuilles de Chibiao 池表; 150 feuilles chacun de jiabiao et de Xuanlian 宣連》 (témoignage sur des faits qui se sont produits l'année précédente ; SHY, zhiguan, 一/起居院, vol. 3, p. 2380 [ce $57: 17]$ ). En 1151 (21 année shaoxing), « on a accepté d'ajouter une part de papier jiabiao et Xuanlian, pour chaque type de 1000 feuilles " (SHY, zhiguan, 一三禮部, vol. 3, p. 2667 [ce $67: 6]$ ). Le jiabiaozhi semble désigner dans ces deux cas une qualité de papier. Toutefois on s'attendrait davantage à ce qu'il s'agisse d'un nom de lieu, puisqu'il est aussi question du biao de Chi et du lian de Xuan, deux localités peu éloignées dont il est également question dans la partie finale de cet article. 
à une qualité de papier pour le revêtement ou le redoublement ${ }^{47}$. Ce papier aurait dû être utilisé pour imprimer des «certificats gagés sur le sel » (yanchao 覧鈔), mais apparemment, en raison de ses caractéristiques, il ne fit pas l'affaire. Il n'est d'ailleurs pas question dans ce cas du sud de l'Anhui, mais d'une production de Xiangzhou 裹州 dans le Nord de l'actuelle province du Hubei :

Au $22^{\mathrm{c}}$ jour du $4^{\mathrm{c}}$ mois (de la cinquième année de l'ère Yuanfeng 1082), la Commission des finances a signifié la chose suivante : "Le décret de la Cour ordonne que 2000000 de ligatures des certificats gagés sur le sel soient distribuées dans le Circuit du bassin du Jing-Yuan [涇原路, correspondant au Shaanxi-Gansu]. Or l'intendance fiscale (zhuanyun si 轉運司) du Shaanxi a dressé un bilan de ses papiers monétaires (chaozhi 鈔紙) et il manque effectivement 480000 feuilles. Si l'on attendait que les préfectures de Shang 商, Guo 虢, etc. procèdent à des achats obligatoires (kemai 科買), évidemment cela aboutirait à un blocage. Nous souhaitons donc utiliser du papier jiabiao de Xiangzhou [dans le Hubei septentrional] de l'entrepôt des objets divers pour en imprimer. » Mais l'empereur a annoté un rescrit indiquant : « Cette qualité de papier, qui n'a jamais été faite pour cet usage, n'est pas pratique ; il convient d'ordonner que la qualité supérieure de papier utilisée ainsi depuis longtemps reste la meilleure pour l'impression. »

Le fait que ce papier ne fit pas l'affaire et que sont emploi ait été envisagé dans d'autres localités ne veut pas dire que, sous les Song, les papiers du Sud-Est ne furent jamais utilisés comme papier-monnaie. Environ vingt ans plus tard, il fut accordé aux régions du Sud-Est, productrices de papier, d'en imprimer à l'usage exclusif de l'administration ${ }^{48}$. Au siècle suivant, Hong Mai 洪邁 (1123-1202) note que l'administration publique fait des billets huizi 會子 à partir de la trentième année de l'ère Shaoxing (1160), lorsque Qian Duanli 錢端禮 (1109-1177), devenu vice-président du ministère des Finances, confie à Huizhou la production de 500000 feuilles (dont les bordures n'étaient pas découpées ${ }^{49}$ ).

47. Elle est évoquée à deux reprises dans le SHY : dans le règlement à propos du sel (yanfa) inclus dans la section shihuo . 四/監法, puis dans les compléments, toujours dans la section shihuo, aux entrées portant sur les certificats gagés sur le sel, yanchao; 補編 ·補遺/食貨四 | 七儖鈔. SHY, vol. 6, p. 6205 (ce $133: 22$ ).

48. Les notes du Song huiyao reviennent sur ce sujet, cette fois au nom du ministère du Cens ( $($ G部') et, plus exactement, de la cour du Palais des revenus (Taifu si 太府孝). On y apprend qu'en 1102 (première année Chongning), outre le « papier monétaire» (chaozhi 鈔紙) des localités septentrionales (Shang 商, Guo 虢, Hezhong 河 11) qui produisaient un papier de qualité grossière (maotou zhi 毛頭紙), on accorda à la préfecture civile et militaire du Sud-Est productrice de papier de fabriquer une catégorie de «papier monétaire » avec engagement d'achat sur trois ans. Les quantités (684000 feuilles) et les dimensions (pour une planche d'impression de $1,7 \times 1,1 \mathrm{chi}$ ) de ce papier furent alors fixées. Il était exclusivement destiné à être versé à l'administration ; toute autre production ou commercialisation était interdite : on fixa à 100 coups de bâtons la peine pour ceux qui viendraient à transgresser cette prescription et à 30 ligatures la récompense pour les délateurs des infractions : cf. SHY, shihuo, 一匹匹/ 覧法; vol. 6, p. 5211-5212 (ce 133 : 34-35). Le maotou zhi est aujourd'hui décrit comme un papier épais, pouvant être utilisé pour la confection des emballages, etc. Il fait partie d'une livraison de papier pour la $12^{\mathrm{e}}$ année Shaoxing (1142), citée plus bas ; voir note 61 ci-dessous.

49. Entrée Guanhui zheyue '户會折閱 (Échange [à perte] des hui de l'administration): Hong Mai,

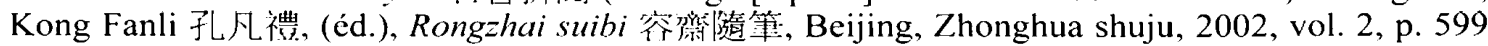
(Rongzhai sanbi 容纃三筆, ch. 14). Ce texte est partiellement cité dans Bao Yilai 鮑军来, Huizhou gongyi 徽州工藝, “Huizhou wenhua quanshu », Hefei, Anhui renmin daxue chubanshe, 2005, ch. 12, p. 125. 
Plus tard, les auteurs du Songshi citent eux aussi l'opération de Qian Duanli, témoignant de la situation aux environs de l'année 1162 (fin du règne de Gaozong) :

Pendant la $32^{\mathrm{c}}$ année du règne a été fixée la loi contre la fausse production de huizi... À l'époque, le papier des [billets] hui venait de Hui 徽 et de Chi 池. Ensuite la production était effectuée à Chengdu 成都, et également à Lin'an 臨安; au début la circulation était limitée au Liangzhe 兩浙, mais après ils ont circulé dans l'ensemble des circuits de la Huai 淮, du Zhe 浙, du Hubei 湖北 et du Jingxi 京西... ${ }^{50}$

Le Song huiyao fait état d'informations concernant l'achat et la distribution d'autres papiers. Le nom de She[zhou] ${ }^{51}$ y apparaît, mais aussi ceux des localités voisines de Xuan 宣 et Chi 池, également situées dans le sud de l'Anhui et appelées à devenir particulièrement célèbres pour leur production de papier. Parfois, les énumérations de produits présentent une succession qui pourrait être liée à l'utilité ou à la valeur du papier ; de même, la quantité de papier de Chi mise à disposition est souvent inférieure à celle de She. Ces quotas nous font supposer que le papier de Chi était plus précieux ou produit dans de plus petites quantités.

De plus, les quantités de biaozhi sont moindres par rapport à celles de dalian 大連, de dachao 大抄 et de xiaochao 小抄. On comprend qu'il y a là aussi unc « hiérarchie » des produits, qui sont souvent cités dans le même ordre. C'est le cas, par exemple, dans les quotas de papier qui sont notés en 1136 (sixième année de l'ère Shaoxing) pour les prescriptions du «Bureau des visites 》 (客省格) et de son bureau subordonné, le «Bureau des quatre directions » (四方館格) : à chaque saison, le premier reçoit 700 feuilles de biaozhi, 1000 de dachao, et 1200 de xiaochao, ainsi que 4 liang de vermillon (朱紅); pour le deuxième, les quotas de ces papiers sont de 200, 800 et 100 feuilles, plus 3 liang de vermillon et, dans les deux cas, les quotas sont augmentés d'un tiers quand la saison compte un mois intercalaire ${ }^{52}$.

Si notre lecture du Song huiyao se limitait aux informations concernant étroitement le territoire de Huizhou, plusieurs dates seraient à retenir : elles sont concentrées dans le premier quart $\mathrm{du} \mathrm{XI}^{\mathrm{e}}$ siècle, puis pendant l'ère Xining (1068-1077) et enfin dans les années 1140 .

- En 1007, ainsi que le Xin'an zhi le relatait (voir plus haut), Shezhou est concerné par une baisse des quotas de papier, en raison peut-être des contrôles voulus par Zhenzong 真宗 qui craignait que les augmentations des bénéfices en cours puissent cacher des prélèvements abusifs ${ }^{53}$.

-En 1011, Wang Qinruo 王欽若 (965-1025) en charge à la Cour des affaires militaires (shumi yuan 樞密院) écrit : “" “e biaozhi de Shezhou, qui est réclamé par toutes les sections de cette Cour, à partir de la première année Dazhong xiangfu (1008) est surveillé

50. SS-SKQS (史部/正史類/宋史/卷一百八十一); Songshi, p. 4406. Ce passage est également signalé par Pan Jixing (1998, p. 205); Pan transcrit aussi un texte de Wu Zimu 吳自牧 (1231-1309) où Huizhou est de nouveau évoqué comme lieu de production de papier pour le huizhi; cf. ibid.

51. Le nom de Shezhou est employé par la préfecture à plusieurs reprises depuis les Sui (589); sous les Song, il est changé en Huizhou en 1121 ; il est donc logique qu'il soit question de Hui dans les trois textes sur les billets huizi que nous venons de lire.

52. SHY, zhiguan, 三五/回方館; vol. 4, p. 3066 (ce $77: 12$ ).

53. C. Lamouroux, 2003, p. 132. Voir aussi plus haut, p. 271. 
rétrospectivement année après année. Actuellement, en dehors des commissaires adjoints, il en reste 118300 feuilles. En encourageant la Commission des finances à contrôler les dépenses pendant un an, on arriverait à réduire la quantité pour cette préfecture." On a agi en conséquence et des émissaires impériaux ont été envoyés au Palais pour faire une déclaration aux subalternes de Zhang Zhi 張質 (944-1017), vice-rapporteur à la Cour ; aussi y a-t-il eu un banquet offert au nom de l'empereur au temple Taiping Xingguo. À ce moment-là, l'empereur se servait de la quantité de papier grand format versée chaque année par Shezhou. Comme les chiffres allaient croissant, c'était extrêmement pénible pour le petit peuple ; afin de le soulager, Qinruo a donc adressé ce mémoire sur la base de ces différentes considérations $"{ }^{54}$. Cette même affaire revient dans l'ouvrage de Li Tao ${ }^{55}$, ainsi que dans la monographie de Luo Yuan, comme nous le verrons plus bas.

- Pendant la cinquième année de Dazhong xiangfu (1012), la préfecture de Kaifeng 開封府 propose des réglementations; il y est question d'un papier jiang 降 mesurant 2,3 chi de longueur, dont la production était confiée aux circonscriptions de She et de Hong 洪 (au Jiangxi, près de l'actuel Nanchang 南昌) ${ }^{56}$.

- Deux ans plus tard (1014), au dixième mois de la septième année de l'ère Dazhong xiangfu, on ordonna de produire du biaozhi à Shezhou. Des fonctionnaires contrôleurs (au niveau de la préfecture) étaient mandatés pour vérifier que les artisans en fabriquent autant qu'il y avait de matériaux, après que ceux-ci avaient été pilés (et blanchis), tandis que les préfets en poste devaient examiner et réceptionner le papier ${ }^{57}$.

- En 1068, il est question des 1000 feuilles de (papier) baihua, recensées aussi dans le Xin'an zhi ${ }^{58}$.

- Pour l'année $1077(?)^{59}$, dans une correspondance d'achats de biens dont la valeur totale est indiquée en argent, sont comptabilisées pour le circuit du Jiangnan oriental 江南東路 3255400 feuilles, provenant de Chizhou, Xuanzhou, Nankang 南康, du Jiangxi 江.西 et de Shezhou. La quantité de cette production est importante quand on la compare avec les autres chiffres relatifs au papier dans le même document, notamment par rapport à ceux du circuit occidental du Jiangnan 江南西路 (1 274000 feuilles) et du circuit septentrional de Jinghu 荆湖北路 (559 550 feuilles) ${ }^{60}$ (Tableau 2).

54. SHY, zhiguan，六/榀密院承旨司 ; vol. 3, p. 2458 (ce 63 : 3). Sur Wang Qinruo et Zhang Zhi, cf. Chang Bide 晶彼得 et al., Songren zhuanji ziliao suoyin 宋人傳記資料索引 I, Taipei, Dingwen shuju, 1977, p. 350-351 et p. 2316.

55. XZCB, ch. 76 (vol. 6, p. 1726-1727): dans ce passage, il semble être question pour la Commission de suspendre pendant un an les approvisionnements de papier afin de réduire les quotas pour la préfecture.

56. SHY, xingfa, 一/禁約; vol. 7, p. 6501 (ce 165:11). Ce même passage fait mention de l'utilisation d'un papier jaune teint de qualité inférieure, ainsi que d'un flottement dans la réglementation suivie.

57. SHY, shihuo 石二/雜物庫; vol. 6, p. 5703 (ce 146:8). Il est parfois difficile d'établir un distinguo entre les caractères bai 白 (blanc) et jiu Fł (piler). Mais de nos jours encore, il arrive que des fibres se retrouvent décolorées à force d'être pilées dans l'eau : les deux caractères finissent alors, en dépit d'un sens différent à l'origine, par désigner quelque chose de comparable.

58. Voir plus haut, les textes traduits aux pages 269 et 271.

59. Je choisis de suivre la version en ligne du SHY (shihuo, 三四/各路產物買銀俉) qui ouvre le passage par cette date. Cependant le texte est très lacunaire; quand on consulte la version imprimée de cette œuvre, on voit que cette date figurait en note ; SHY vol. 6, p. 5407 (ce 138 : [3]8). Les autres dates sur la même page, également en note, se réfèrent aux mêmes années : à nouveau la $10^{\circ}$ année Xining (1077), puis la première et deuxième année de l'ère Yuanfeng (1078-1079).

60. Dans ce cas, les listes de qualités de papier qui sont données soulèvent la question de leur rationalisation. Ce problème se retrouve aussi dans les informations du Xin'an zhi (voir note 63). Nous avons été ainsi incitée à cet excursus pour comparer d'autres sources, sans pouvoir arriver à des conclusions 
- En 1142-1143 (fin de la $12^{\circ}$ année Shaoxing), pour le Bureau des palanquins impériaux (Yunian yuan 御替院) sont prévues 50 feuilles de biao de Chi[zhou], 100 de biao de She[zhou] de grandes dimensions et 200 de petites dimensions, ainsi que 500 feuilles de maotouzhi ${ }^{61}$.

Avant cette dernière note, nous pouvons compter pour les années 1080-1085 mille feuilles de papier supplémentaires comptabilisées pour She dans la liste des contributions tributaires incluses dans le Yuanfeng jiuyu $\mathrm{zhi}^{62}$.

Ces informations réunies nous permettent de mieux comprendre le sens d'un dernier passage du Xin'an $z h i$, dans lequel Luo Yuan décrit les quantités de tributs annuels versés en papier (shanggong zhi 上供紙) : il est bien évident que ce tribut est important en quantité, et que le papier est le support des textes de l'administration qui, nous l'avons lu, s'en sert à différents niveaux, d'une façon assez codifiée. La liste précédente nous permet de saisir aussi les limites de notre source locale, car les données du Xin'an zhi sont finalement restreintes et certes moins nombreuses que ce qu'on aurait dû conserver dans les archives de la préfecture. Dans le Xin'an zhi, nous lisons encore qu'il existait différentes sortes de papier :

Sont versées en tribut sept qualités de papier, pour un montant annuel de 1448632 feuilles. Les sept qualités sont : la changyang 常様, la jiangyang 降様, la dachao 大抄, la jingyun sanchao 京運三抄 et la jingyun xiaochao 京運小抄. En dessous du sanchao, il y a le papier pour achats mai(zhi) 買(紙) et le papier pour rédiger les mémoires zouzhi 奏紙, ainsi ils forment bien sept sortes ${ }^{63}$. Il y a, en plus, des quotas annuels de papier

probantes. En fait, seulement trois qualités de papier semblent se dégager pour Shezhou en se basant sur la ponctuation donnée dans la version en ligne du SHY (制州詔紙降樣, 常樣大抄、三抄連紙). Mais on comprend qu'elles sont probablement plus nombreuses en comparant cette version avec d'autres textes. D'ailleurs l'expression zhaozhi 詔紙 (papier pour édits?) est aussi utilisée pour l'année 1013, où il est question de l'usage d'un zhaozhi blanc ou jaune selon les postes et les grades de l'administration concernés : SHY, zhiguan, 六/翰林院; vol. 3, p. 2520, 2521 (ce 63 : 49). Si nous schématisons les informations du SHY pour cette période, en les comparant à celle de l'ère Yuanfeng du YJZ, nous obtenons les données présentées au Tableau 2.

61. SHY, zhiguan 一九/禦婑院; vol. 3, p. 2819 (ce 71: 18). Voir plus haut, note 48 .

62. Voir aussi note 37 et Tableau 2.

63. Nous avons été obligés de trancher sur ce point, sans savoir si nous faisions vraiment le bon choix. En fait, une partie de ces noms de papiers, quand nous regardons ce passage dans les versions existantes du Xin'an zhi (qui, rappelons-le, datent des Qing) dont celle du Siku quanshu, ou dans celle incluse dans le Huizhou fuzhi (qui est une compilation plus récente que le Xin'an zhi, mais qui nous est parvenu dans des versions plus anciennes que la première monographie), sont à chaque fois écrits différemment. Ici, nous avons utilisé la version du XAZ-SKQS, chapitre 2, ponctué d'une façon parfois différente de celle utilisée par les auteurs chinois de nos jours. Mais la cohérence manque et nous ne pouvons pas exclure que le texte soit corrompu; d'ailleurs dans le HF-HZ, un caractère a été introduit et il y est question de zhemai zouzhi 折買奏紙. Si nous consultons le XAZ-F, repris dans la version moderne ponctuée XAZ-EC, ou encore dans des citations contemporaines, les sept papiers sont distingués en changyang 常様,jiangyang 降様, dachao 大抄,jingyun 京運, sanchao 三抄,jinglian 京連 et xiaochao 小抄; ainsi les qualités sont en nombre suffisant, et le papier pour rédiger les mémoires est (acheté ?) à part. Dans les deux volumes du « Huizhou wenhua quanshu » (2005), les auteurs du volume sur les techniques reprennent l'énumération (Hu Huagai et Zhang Binglun, Huizhou keji, p. 267), tandis que Bao Yilai (Huizhou gongyi, 2005, p. 125) préfère ne pas donner de liste, mais interprète qise 二色 dans le sens de « sept couleurs » et imagine donc du papier teint. Cette lecture peut avoir été inspirée par des textes comme celui du Hanlin zhi (voir plus haut note 13), dans lequel on découvre que la couleur fonctionnait 
pour les billets commutables en monnaie (zhiqian zhi 折錢紙) qui est employé comme des [feuilles] dachao commutables en achats (zhemai dachao 折買大抄). Tout ce qui rentre dans les normes est envoyé pour être entreposé au Trésor de Gauche. Il y a encore le papier pour les académiciens (學士院紙), celui pour la Division de droite [右漕紙 du ministère du Cens] ainsi que celui pour les papiers monétaires gagés sur le sel et le papier des certificats du thé (yanchao chayin zhi 櫺鈔茶引紙), dont les quantités ne sont pas chiffrées. Les quantités de papier de grandes dimensions versées chaque année (sui gong dazhi 歲供大紙) par la préfecture de She 㰸州 ont augmenté depuis le sixième mois de la quatrième année Dazhong xiangfu (1011), ce qui, à cause de leur ampleur, est devenu un souci pour la population...

Le texte s'achève par la reprise de l'affaire de Wang Qinruo et Zhang Zhi, citée plus haut ${ }^{64}$, concernant la tentative de réduire l'utilisation du papier produit à Xin'an. Luo Yuan précise aussi que l'on ne sait pas à quel moment les nouveaux quotas ont été établis.

Les mesures de ce type semblent se succéder. Li Tao nous informe en effet qu'à la fin de la deuxième année du règne Tiansheng (1024), sous Renzong, une décision concernant la réduction d'un tiers pour les tributs en papier de Shezhou est prise par la Commission des finances ${ }^{65}$.

\section{Les trois trésors du lettré}

Si les listes de quantités de papier récupérées et distribuées par l'administration restent des données assez stériles, ces passages attestent dans l'ensemble d'une production locale importante et variée. On réalise cependant qu'il n'y pas de distinction entre les noms des qualités de papier et les noms que l'on donne au papier pour son emploi ou en fonction des bureaux où il est utilisé : nous sommes face à une catégorisation certainement aléatoire, qui est aussi déroutante que répandue dans ce type de littérature ancienne où les juxtapositions sont nombreuses, ce qui a d'ailleurs fini par inspirer nos écrivains et philosophes ${ }^{66}$.

Cependant, ce sont les qualités chao 抄, notées dans plusieurs des citations examinées, qui méritent notre attention, car ce caractère sert aussi à désigner la confection de la feuille de papier en grand (大抄) ou en petit (小抄) format, ou en répétant l'opération à plusieurs reprises (三抄 en triple épaisseur ?) ${ }^{67}$. On en déduit donc que les formats

comme élément de distinction pour des papiers à usages codifiés. Il existe une importante littérature à propos des papiers colorés, surtout pour la dynastie Ming. Mais ici, il nous semble préférable d'interpréter se 色 comme «sorte/qualité » de papier (ce qui n'exclut pas complètement la possibilité d'une variété de couleurs). Dans le HZFZ-HZ, ch. 2, p. 52r, il n'est pas question de jingyun 運, mais de jinglian 連, ce qui est aussi logique au vu des données du SHY, réunis dans le Tableau 2.

64. Voir plus haut, notes 54 et 55 .

65. Voir XZCB, ch. 102 : vol. 8, p. 2396. Ce moment correspondrait aux premiers programmes de réforme pour contenir les dépenses publiques, que relate aussi le Songshi; voir C. Lamouroux, 2003, p. 137-138.

66. La fameuse citation d'《 une certaine encyclopédie chinoise » inventée par Borges qui est donnée par Michel Foucault dans l'introduction de Les mots et les choses, une archéologie des sciences humaines (Paris, Gallimard, 1991, p. 1) dépasse largement les soucis et les recherches des sinologues; à ce propos voir Extrême-Orient, Extrême-Occident, 1988, n 10 : «Effets d'ordre dans la civilisation chinoise (rangements à l'œuvre, classifications implicites) ».

67. Selon Pan Jixing (1998, p. 251), il ne s'agirait pas de replonger plusieurs fois la forme, mais de prendre plus de pâte à l'aide d'une immersion plus profonde, ou d'obtenir un papier à plusieurs couches ensuite lors du séchage, en superposant deux ou trois feuilles encore humides pour n'en confectionner 
étaient eux aussi fixés. Nous ne savons pas si ces papiers, « grand » et " petit » format, ainsi que le papier de grandes dimensions (dazhi 大紙) de 1011 dont il est également question dans le texte précédent, pourraient être des évolutions des papiers « grands » et « petits » formats du passé. Pour l'époque Jin 晋 (266-420), Su Yijian 蘇易間 (958-996) en avait décrit les dimensions un siècle et demi plus tôt dans son Wenfang sipu 文房四譜, le Recueil des quatre trésors du lettré. À titre indicatif, selon l'équivalence établie par Pan Jixing, les mesures étaient de $31,3 \times 43,4 \mathrm{~cm}$ pour le grand format, et de $22,9 \times 33,7 \mathrm{~cm}$ pour le petit ${ }^{68}$, ce qui est finalement assez conforme aux nombreux papiers d'ouvrages calligraphiques des Song qui ont été conservés jusqu'à nos jours. D'autres papiers, un peu plus grands, auraient été destinés à accueillir l'impression de planches d'environ $34 \times 53 \mathrm{~cm}$ en $1084^{69}$. Par ailleurs, le format aurait augmenté au cours des siècles. Nous avons rappelé les dimensions d'un papier pour l'administration, long d'environ $72 \mathrm{~cm}$, fabriqué entre She et Hong vers 1012. On peut encore ajouter un papier pour décrets (zhaochi 詔勑) d'environ 40 × $62 \mathrm{~cm}$, cité dans le Qingyuan tiaofa shilei 慶元條法事類 (Articles de loi de l'ère Qingyuan [1195-1200] classés par catégories), le plus grand format utilisé à la fin du $\mathrm{XII}^{\mathrm{e}}$ siècle dans l'administration. D'autres papiers auraient pu être fabriqués dans un format très long, afin d'être ensuite découpé en plusieurs feuilles destinées à la vente ${ }^{70}$.

Mais il existait également un papier bien plus grand, dont il est question dans un texte de Su Yijian repris dans la monographie de Luo Yuan. En fait, les parties finales du $X i n$ 'an zhi révèlent son ancienneté : malgré un nombre important de biographies, on n'y trouve pas de personnages célébrés pour leur savoir-faire technique, comme c'est parfois le cas dans certaines monographies des Ming et des Qing. De plus, même si cette section biographique avait existé, il est probable que le papier n'y aurait pas été évoqué. Dans la littérature des lettrés, les papiers les plus précieux sont décrits et appréciés, mais à quelques exceptions près, les informations sur le papier ne sont pas «personnalisées » ${ }^{71}$. Ce fut en revanche le cas d'autres produits locaux, tels les bâtons d'encre et toute une kyrielle de noms subsiste, à commencer par ceux de Li Chao 李超 et de son fils Li Tinggui 李廷珪. En fuyant le Nord du pays au $\mathrm{X}^{\mathrm{e}}$ siècle, ils auraient introduit la manufacture d'encre dans

qu'une seule. De nos jours, il semble que les différentes méthodes soient utilisées ; communication personnelle de Claude Laroque, que je remercie ici.

68. Nous avons choisi d'utiliser ces équivalences, mais en réalité on pourrait en établir d'autres, à cause d'une ambiguïté entre le cun et le fen dans le texte. Dans son ouvrage, Pan Jixing (ibid. p. 127) rationalise la transcription, en prenant des libertés avec le texte à propos des nombres décimaux. Si l'on suit à la lettre la $38^{\circ}$ entrée du chapitre 4 du Wenfang sipu (1936), sous les Jin, le grand papier mesurait 1,08 chi de long et entre 1,03 et 1,4 chi de large ; le petit, lui, mesurait 0,95 chi de large et 1,4 chi de long, ce qui rendrait finalement les dimensions des deux types de papier quasi identiques.

69. Ces dimensions sont calculées sur la base des informations données à la note 48 et d'une l'équivalence de $1 \mathrm{chi}$ pour $31,2 \mathrm{~cm}$; cela rendrait cependant les planches très grandes pour du papier-monnaie, plus ce que l'on trouve dans les exemples présentés par H. U. Vogel, Marco Polo Was in China, New Evidence from Currencies, Salts and Revenues, Brill, Leiden-Boston, 2013, p. 103-105 et suiv.

70. Toutes ces informations sont données par Wei Huaxian, 2006, p. 137 et 178. Selon cet auteur, les grands formats seraient le fruit d'un travail collectif, dépassant le cadre d'une production familiale restreinte : plus grand est le format, plus il témoignerait d'un système de travail associatif et structuré ; ibid. p. 141-142; voir aussi note 201 .

71. Si l'acquis technique est souvent associé à l'intervention d'un personnage mythique ou historique, les compétences techniques des artisans sont parfois transmises par des textes biographiques, inclus dans les histoires locales ; sur cet aspect qui, à quelques exceptions près (voir plus bas, notes 174 et 195), fait normalement défaut pour le papier, voir les contributions de Martina Siebert et de Martin Hofmann dans D. Schäfer, 2012, p. 253-297. 
cette région montagneuse qui abritait les spécimens de sapins nécessaires à la production d'une suie de qualité.

Le Xin'an zhi n'a pas non plus de section bibliographique, qu'il s'agisse d'une énumération des titres d'ouvrages publiés localement par des lettrés originaires de la région, ou bien d'une anthologie des extraits sélectionnés correspondant normalement aux textes introductifs desdits ouvrages, comme cela se pratique normalement dans les monographies locales. Mais, à propos du papier dans le chapitre 10 , Luo Yuan utilise la méthode de la citation, ce qui ne se reproduit pas toujours dans les monographies suivantes. La section porte sur le papier, les pierres à encre et les encres. Il n'y est pas question des pinceaux qui, à l'époque, sont plutôt des produits provenant de la région voisine. Il n'est donc pas surprenant de lire quelques pages plus loin : «On dit qu'à Shezhou sont produits les quatre trésors du lettré qui sont le papier, l'encre, les pinceaux et les pierres à encre; mais en réalité ils ne sont que trois car à Shezhou on ne fait pas de pinceaux, qui eux viennent de Xuanzhou ${ }^{72}$. D'autres sources contemporaines ou plus tardives attribuent en effet la production de pinceaux à Xuan et celle des autres produits à $\mathrm{Hui}^{73}$.

Dans la section du chapitre $10 \mathrm{du}$ Xin'an zhi sur le papier, nous lisons ensuite deux extraits du Wenfang sipu de Su Yijian à propos de ces papiers spéciaux, en dimension ou en utilisation ${ }^{74}$ : ce sont de produits sophistiqués nécessitant des compétences techniques indéniables. Il est probable que les différentes étapes correspondaient à des interventions spécialisées, mais malheureusement les divers intervenants ne sont pas présentés comme tels dans ce texte, même si nous pouvons imaginer " ouvreurs ", " coucheurs " ou «vireurs » occupés autour de l'énorme feuille décrite dans le premier texte :

Entre Yi et She, il y a beaucoup de papiers d'excellente qualité, dont ceux appelés ningshuang et chengxin. Il y a également un papier de grande longueur, dont la taille est de 50 chi. Les gens de She préparent du mûrier à papier pendant plusieurs jours. Ensuite, ils le plongent dans l'eau dans une [cuve allongée en forme de] coque. Des dizaines de personnes lèvent la [pâte avec une] forme [pour confectionner une feuille], tandis qu'à côté quelqu'un marque le rythme avec un tambour. Ensuite [le papier] est séché au feu, après avoir été installé sur une grande claie de bambou. On ne l'étale pas sur des murs. On obtient des feuilles toutes fines et régulières du début à la fin.

Ceux qui vivent dans les montagnes utilisaient du papier pour faire des vêtements. Ceux qui respectent (la doctrine du) Bouddha disent qu'il s'agit d'une façon de s'habiller sans utiliser les vers à soie. Ce sont en fait des vêtements très chauds : celui qui porte ces vêtements a, en moins de dix ans, le visage jauni et l'énergie reduite, dans le souci

72. Cette citation est tirée du Bishu luhua 避暑録話 (Notes prises dans ma villa d'été, vers 1135 ) de Ye Mengde 葉罗得 (1077-1148) : voir le texte inclus dans le SKQS, juanshang, p. 16-17. Dans ce même passage, Ye se plaint aussi de la qualité du papier contemporain, inadaptée pour obtenir une écriture à l'encre aux noirs bien profonds.

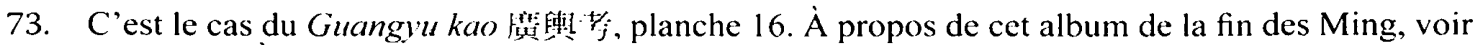
plus bas, p. 298. A propos du YJZ, voir aussi plus bas, note 145.

74. XAZ-SKQS ch. 10. Hu et Zhang (Huizhou keji, p. 268) font aussi référence à un papier spécial

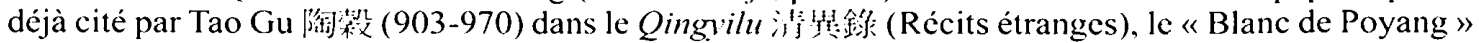
(Poyang bai 翻陽备1) grand comme un pi de tissu (pour cette unité de mesure, voir plus haut, note 35), en avançant l'idée qu'il était produit à Huizhou. Or, cette attribution non seulement semble un peu forcée, mais l'ouvrage même est douteux; cf. É. Balazs, Y. Hervouet, A Sung Bibliography, Hong Kong, The Chinese University Press, 1978, p. 320. 
de contenir ses passions. De plus, ils ne conviennent pas au lavage. [Les vêtements en papier] ne laissent pas pénétrer le vent et le souffle intérieur ne peut pas en sortir. On dit que pour faire des vêtements de papier, pour cent feuilles, il faut ajouter une once de hutao 胡桃 (noix, Juglans regia L. [?]) et une once de ruxiang 乳香 (résineux du boswellia - résine pour fabriquer l'encens ${ }^{75}$ ). On fait bouillir [les feuilles pour absorber le liquide pendant la cuisson], ou on les fait cuire à la vapeur, ce qui est encore mieux. Pendant la cuisson à la vapeur on arrose constamment de résine et d'autres liquides. Dès qu'elles sont cuites, on les fait sécher à l'ombre ; en utilisant un bâton de bambou, on en fait un rouleau dans le sens de la largeur, ce qui permet de presser facilement [une feuille en la faisant rouler ?] ; et quand il y a un défaut, on le raccomode minutieusement. De nos jours, dans les préfectures de She et de Yi, certains font des vêtements en papier, dont une pièce peut être presque aussi grande qu'une porte à deux battants. Actuellement, les fonctionnaires qui partent en mission utilisent ces vêtements car ils profitent de leur résistance au vent dans les moments de grand froid. Tao l'ermite (陶鿵居, Tao Hongjing 陶弘景, 456-536) disait que les gens de Wuling 武陵人 (Changde 常德, au Hunan ?) faisaient des vêtements en écorce de mûrier à papier, gupi $i$ 穀皮, qui étaient extrêmement résistants.

Cette production d'objets en papier est bien connue en Chine : des pic̀ces anciennes ont été retrouvées ${ }^{76}$. Les témoignages écrits sont particulièrement nombreux pour la dynastie des Song, à tel point que le papier est parfois présenté comme un produit de substitution, notamment aux tissus, pour répondre à la demande provoquée par la croissance démographique ou comme alternative moins onéreuse ${ }^{77}$. Mais notre texte montrerait que des catégories spécifiques de personnes étaient concernées, qu'il s'agisse de religieux ou d'administrateurs-voyageurs, et non le commun des mortels.

Parmi les citations incluses dans le Xin'an zhi, il y a encore ce bref extrait issu du Wenfang sishuo 文房四説, ouvrage attribué à Cai Xiang 蔡襄 $(1012-1067)^{78}$ :

Le papier du Chengxin tang 澄心堂 sous les souverains Li 李 [des Tang du Sud à Nankin, 937-975] était un produit de premier choix, qui venait des préfectures de Chi et de She du Jiangnan. On ne fait plus actuellement de produits d'excellente (qualité).

75. L'utilisation de la résine d'oliban est attestée dans les pratiques montagnardes pour fabriquer de la colle et des cartons; Schmitt, 2011, p. 272. Il ne serait donc pas étonnant de la trouver utilisée ici afin de renforcer cet épais papier, mais le problème est que, logiquement, ce serait un produit importé (Fèvre \& Métaillé, 2005, op. cit., p. 379). Quant à l'expression hutao 胡桃, selon la même source (p. 167), il s'agit d'un synonyme de hetao 核桃, et désigne donc du noyer. Mais Pan Jixing (1998, p. 242), en commentant un texte du $\mathrm{Xv}^{\mathrm{e}}$ siècle, dit que hutaoteng 胡桃藤 serait un synonyme de yangtaoteng 楊桃藤 (Actinidia chinensis). La matière extraite des tiges de cette plante (le kiwi) augmente la cohésion des fibres du papier et peut donc logiquement avoir aussi servi dans la fabrication de ce type de papier pour vêtements. Voir aussi plus bas p. 294, note 114.

76. Selon Tsien Tsuen-Hsuin, de tels vêtements ont été conservés au Japon; en Chine, il existe des objets retrouvés dans les sépultures, ainsi que de nombreux témoignages écrits (Tsien, 1985, op. cit., p. 110-113). Plus bas, nous parlerons des rideaux et des parasols.

77. Wei Huaxian, 2006, p. 162-175.

78. Ce texte bref qui souvent n'est pas répertorié dans les bibliographies traditionnelles à propos de Cai Xiang, est aussi en ligne : http://site.douban.com/111253/widget/notes/1317348/note/128255594/ (consulté le 2/10/2013). Au premier paragraphe, le terme Shujian 虫茂 désigne des papiers de bonne facture produits au Sichuan. 
Les papiers à lettres de Shu (蜀侥 Shujian) ne durent pas longtemps et les autres ne sont pas des produits de qualité...

Le papier de Jixi dans la préfecture de She est un nouveau produit qui a hérité (des qualités) de celui du Chengxin tang et son éclat dépasse (celui du papier ancien). Mais de nos jours, la plupart du papier vient du Sud, comme le papier de Wutian 烏田, Gutian 古田 (Fujian) et Youquan 由拳 (Zhejiang), ainsi que de Wenzhou 温州 et Huizhou 惠州. Tous sont devenus célèbres en l'imitant, tandis que celui de Jixi n'est même pas parvenu à franchir la porte (de la célébrité...).

Ce dernier paragraphe ne comporte aucune mention quant à son auteur ou à l'ouvrage cité, mais il semble bien être la continuation du précédent. Après la liste et l'éloge des pierres à encre produites à Wuyuan, il est question des encres : c'est grâce aux meilleurs sapins du Huangshan qu'on a pu obtenir des produits aussi précieux que ceux de Li Tinggui. Et le texte s'achève par des considérations qui sont aussi d'ordre socio-économique :

Ainsi, parmi les artisans, beaucoup sont de pauvres gens et, de ce fait, (motivés par) la recherche du profit, ce qui explique qu'ils n'atteignent pas le niveau. Récemment les " gens du Dao " savent chauffer la suie (pour la ramollir afin de la travailler), ils sont envoyés sur le Huangshan pour obtenir du noir de fumée, il [leur] faut quelque chose d'excellent. En raison du caractère exceptionnel de ces trois produits [papier, encre et pierre à encre] de Shezhou, seuls les amateurs qui disposent de grosses ressources peuvent parvenir (à s'en procurer); mais face au pouvoir de l'administration, personne n'y parvient...

D'autres textes de la période Song pourraient être ajoutés à ce paragraphe. Cependant, pour la plupart, ils concernent la production associée au nom du Chengxin tang, et sont donc indirectement associés à notre localité.

En conclusion de cette présentation à propos de la période des Song, nous pouvons rappeler une dernière " facette » de l'histoire du produit, concernant son commerce et son utilisation. Dans des années relativement proches de la compilation du Xin'an zhi, Hong Mai écrit le Yijian zhi 夷堅志 (Collection des nouvelles merveilleuses de Yijian). Cet ouvrage, tout en étant littéraire, est considéré comme une source riche d'informations sur la société de l'époque. Ainsi Wei Huaxian le cite, car on y trouve le nom d'un marchand de papier installé près d'un pont de She, Zhu Qing 朱慶. C'est un cas qu'il prend en exemple de la commercialisation privée du produit. Nous n'avons pas de raison de douter de l'existence d'un tel négoce dans la préfecture. En revanche, s'il est vrai que le nom de Zhu Qing est présent dans une version du Yijian zhi, l'histoire est fantas-

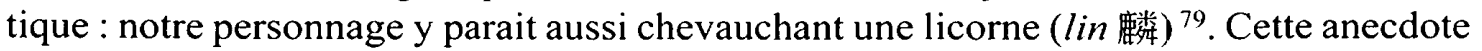
peut être mise en relation avec d'autres passages inclus dans le Yijian zhi, témoignant de

79. Voir Wei Huaxian, 2006, p. 146 et le texte de Hong Mai disponible en ligne (consulté le 16/04/2014): http://blog.sina.com.cn/s/blog_6a5826b60102e 2 w8.html. Il scmblc que l'édition du Yijinzhi imprimée en 1927 par la Commercial Press, conservée en Chine mais non à Paris (et que je n'ai pas encore pu vérifier), contiendrait la section supplémentaire du Yijian zhibu 夷堅志補 où se trouverait ce passage, sous le titre « Jinyuan tong 》 金源洞. 
l'utilisation rituelle du papier comme monnaie d'offrande (zhiqian 紙錢) ${ }^{80}$. On pourrait alors se demander quelles ont pu être les vraies activités d'un tel personnage. Si l'on se base sur ce que nous connaissons de nos jours, la production et la vente de ces papiers s'associe parfois à l'offre de services d'ordre religieux : à une extrapolation des données pour une lecture étroitement économique du récit, comme le fait Wei Huaxian, il faudrait associer une lecture plus nuancée, et s'interroger sur l'existence du magasin et la vraie nature de ses activités ${ }^{81}$.

Cette histoire revient dans un recueil presque contemporain (vers 1150), le Mozhuang manlu 墨莊漫錄 (Notes prises au hasard dans la villa des encres) de Zhang Bangji 張邦基 qui est connu comme bibliophile. Selon ce texte, il n'est plus question de vente, et l'expression utilisée est d'ailleurs obscure ${ }^{82}$. Ainsi, nous sommes encore une fois confrontés à la nature des sources littéraires qui nous montrent certes des pistes intéressantes, sans cependant nous renseigner d'une façon tout à fait certaine.

Une dernière voix que l'on peut inclure parmi les témoignages de la fin des Song est celle de Chen You 陳標, jinshi en 1190, réputé pour sa calligraphie. Dans le « Discours sur les papiers » (Lun zhipin 論紙品) inclus dans son Fuxuan yelu 負暄野錄 (Notes officieuses prises en s'exposant au soleil $)^{83}$, on lit que pour le papier yuban de Xin'an, la couleur est d'un blanc luisant et la matière extrêmement molle, ainsi les fonctionnaires le traitent avec de la colle/amidon pour l'utiliser; le papier est alors brillant et solide et sa longue conservation est assurée sans devoir le faire cuire à la vapeur pour éliminer les mites ; en dehors de la conservation, ce traitement nous fait penser à la préparation d'un papier dit «cuit » (shuzhi 熟紙), en opposition à un papier « cru » (shengzhi 生紙), exempt d'apprêt pour mieux recevoir l'encre. Sous les Yuan, Fei Zhu 費著 (1303-1363 environ) parle des papiers de Hui et de Chi, car des productions similaires à celles de ces endroits furent développées au Sichuan ${ }^{84}$, et Cao Zhao 曹昭 (1330-1400 environ) cite le papier de She dans les termes exacts du Xin'an $z h i^{85}$.

80. Voir, par exemple, l'histoire d'une veuve qui faisait brûler des morceaux ce papier, dans la version du Yijian zhi incluse dans le SKQS (夷堅志/甲卷八, 鄂渚王媼). Cet exemple est cité par Hou Ching-ling, Monnaies d'offrande et la notion de trésorerie dans la religion chinoise, Paris, Collège de France - IHEC, 1975, p. 103.

81. Autrement dit, ce cas peut bien être cité comme exemple d'un point de vente (吾即 災 橋瞉紙 朱慶也), mais encore faudrait-il vérifier où se trouvait le pont près duquel Zhu Qing aurait pratiqué son négoce. Étant donné que les faits sont situés dans la circonscription de She, le territoire est vaste. Difficile ainsi de considérer ce texte comme un témoignage direct à propos d'un processus précis de commercialisation, urbaine et organisée, d'une production papetière paysanne locale ; mais le récit fait évidemment état de pratiques courantes.

82. C'est au chapitre 3 de ces miscellanées que l'on retrouve l'histoire où la phrase est formulée autrement (吾即 歌州某橋南停紙 朱慶也) : voir le Mozhuang manlu dans le SKQS.

83. Ce texte disponible en ligne est cité dans les compilations de savants locaux; consulté le 03/10/2013 : http://zh.wikisource.org/zh-hant $\% \mathrm{E} 8 \% \mathrm{~B} 2 \% \mathrm{~A} 0 \% \mathrm{E} 6 \% 9 \mathrm{~A} \% 84 \% \mathrm{E} 9 \% 87 \% 8 \mathrm{E} \% \mathrm{E} 9 \% 8 \mathrm{C} \% 84$.

81. Fei Zhu, Shujian pu 蜀片戔譜, «Meishu congshu », Shanghai, 1936, vol. 3-5, p. 244-245. À propos du Sichuan, voir aussi plus haut, notes 12, 22, 45. Le fait que les papiers de l'Anhui aient été utilisés au Sichuan montre que ce produit circulait, même entre des régions qui en étaient toutes deux productrices.

85. Cao Zhao et al., Xinzeng gegu yaolun 新增格古要論, “Congshu jicheng chubian », Shanghai, Shangwu yinshuguan, 1939, ch. 9, p. 154. 
Tableau 1 : Papiers de Hui, période Tang - Song * d'après des textes des époques Song et Yuan (cités plus haut, essentiellement aux pages 271-283)**.

\begin{tabular}{|c|c|c|c|c|c|c|c|c|}
\hline $\begin{array}{l}\text { Ouvrages } \\
\text { consultés (à } \\
\text { droite) }\end{array}$ & \multirow{2}{*}{$\begin{array}{l}\text { Xin Tangshu } \\
\text { 新唐書 }\end{array}$} & \multirow{2}{*}{$\begin{array}{l}\text { Wenfang sipu } \\
\text { 文房四譜 (cité } \\
\text { dans Xin'an zhi, } \\
\text { ch. 10) }\end{array}$} & \multirow{2}{*}{$\begin{array}{l}\text { Xin'an zhi } \\
\text { 新安志, } \\
\text { ch. } 2 \text { et } 10\end{array}$} & \multirow{2}{*}{$\begin{array}{l}\text { Fangyu } \\
\text { shenglan } \\
\text { 方舆勝覽 }\end{array}$} & \multirow{2}{*}{$\begin{array}{l}\text { Shanlu 剡錄, } \\
\text { ch. } 7\end{array}$} & \multirow{2}{*}{$\begin{array}{l}\text { Song huiyao } \\
\text { 宋會要 }\end{array}$} & \multirow{2}{*}{$\begin{array}{l}\text { Fuxuan yelu } \\
\text { 負暄野錄 }\end{array}$} & \multirow{2}{*}{$\begin{array}{l}\text { Rongzhai suibi } \\
\text { 容齋隨筆 }\end{array}$} \\
\hline $\begin{array}{l}\text { Noms des papiers } \\
\text { (ci-dessous) }\end{array}$ & & & & & & & & \\
\hline maiguang 麥光 & $\checkmark$ & & $\checkmark$ & $\checkmark$ & & $\checkmark$ & & \\
\hline baihua 白滑 & $\checkmark$ & & $\checkmark$ & $\checkmark$ & & $\checkmark$ & & \\
\hline bingyi 冰翼 & $\checkmark$ & & $\checkmark$ & $\checkmark$ & & $\checkmark$ & & \\
\hline $\begin{array}{l}\text { ningshuang } \\
\text { 凝霜 }\end{array}$ & & $\checkmark$ & $\checkmark$ & $\checkmark$ & & & & \\
\hline chengxin 澄心 & & $\checkmark$ & $\checkmark$ & & & & & \\
\hline longxu 龍䰅 & & & $\checkmark$ & $\checkmark$ & & & & \\
\hline $\begin{array}{l}\text { qiaobing[zhi] } \\
\text { 敲冰[紙] }\end{array}$ & & & $\checkmark$ & & $\checkmark$ & & & \\
\hline yuban 玉版 & & & & & & & $\checkmark$ & \\
\hline $\begin{array}{l}\text { [da]lian[zhi] } \\
\text { [大]連[紙] }\end{array}$ & & & & & & $\checkmark$ & & \\
\hline dazhi 大紙 & & & $\checkmark$ & & & & & \\
\hline changyang 常様 & & & $\checkmark$ & & & $\checkmark$ & & \\
\hline jiang yang 降様 & & & $\checkmark$ & & & $\checkmark$ & & \\
\hline
\end{tabular}

* Ce tableau peut être comparé avec celui établi pour l'ensemble du pays par Wei Huaxian (2006, p. 126-128), où régne une certaine confusion entre les catégories (basées sur les matériaux de production, l'utilisation ou la dénomination des papiers). Le même problème est aussi manifeste pour des sources concernant notre région, comme nous l'évoquons aux notes 60 et 63 .

** On peut constater que les noms cités dans le XAZ sont les plus nombreux, suivis de ceux qui figurent dans le SHY. 


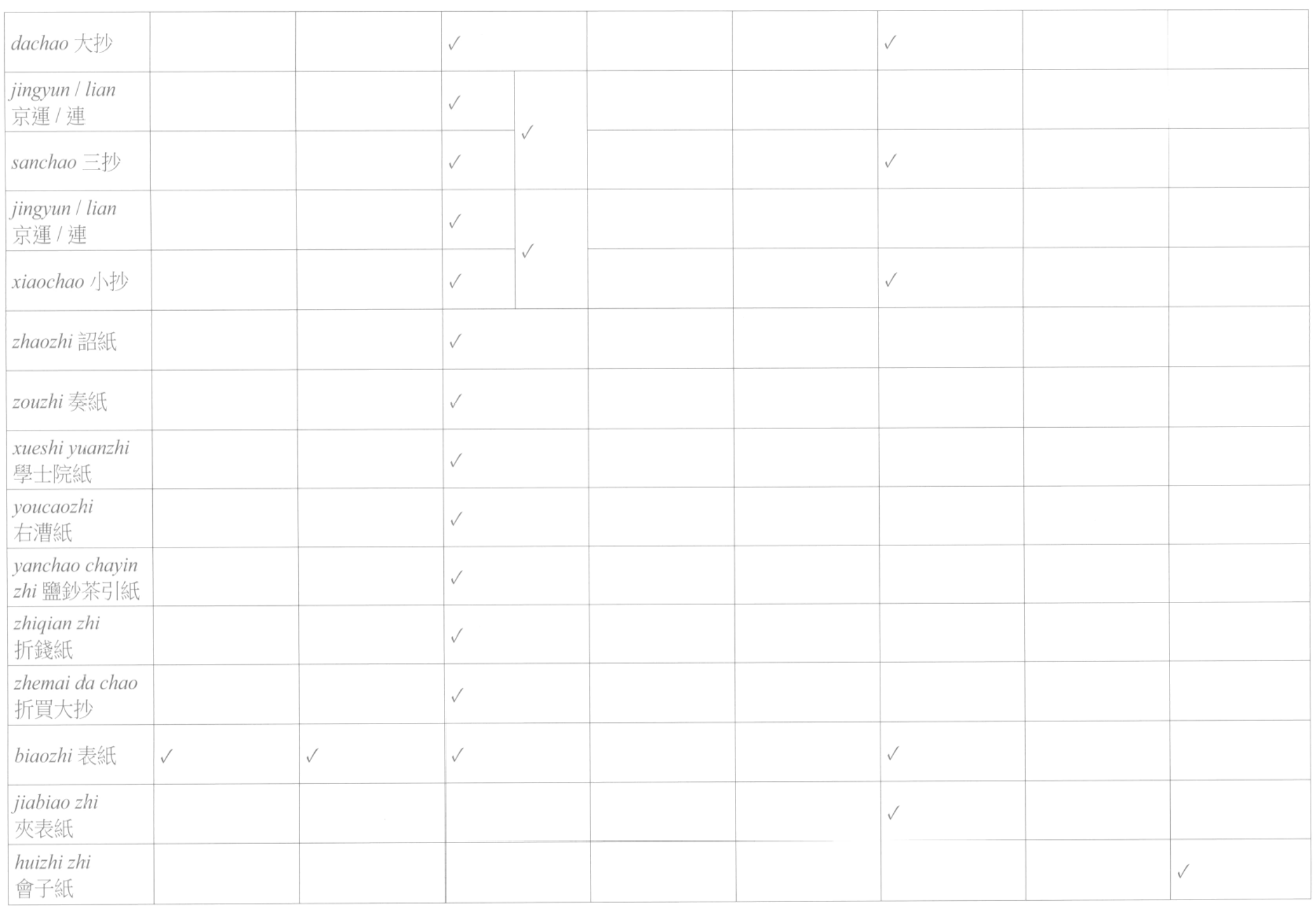


Tableau 2 : Informations sur les papiers produits [pour l'année 1077 ?] figurant dans le SHY; à titre de comparaison, dans la colonne de droite, quelques chiffres donnés dans le YJZ (vers 1080) pour cette même zone géographique

\begin{tabular}{|c|c|c|c|c|c|c|c|c|}
\hline \multicolumn{8}{|c|}{ SHY - différentes qualités de papier selon les localités } & \multirow{3}{*}{\begin{tabular}{|l|} 
YJZ \\
Papier \\
local \\
offert en \\
tribut 土貢 \\
紙一千張 \\
\end{tabular}} \\
\hline \multirow[t]{2}{*}{ Localités } & \multicolumn{7}{|c|}{ Qualités et quantités de papier } & \\
\hline & $\begin{array}{l}\text { (changyang) da } \\
\text { chao lianzhi } \\
\text { [常樣]大抄犝紙 }\end{array}$ & $\begin{array}{l}\text { (changyang) } \\
\text { sanchao lianzhi } \\
{[\text { 常樣] 二抄連紙 }}\end{array}$ & $\begin{array}{l}\text { Lianzhi } \\
\text { 連紙 }\end{array}$ & $\begin{array}{l}\text { Dachao } \\
\text { [表紙]大抄 }\end{array}$ & \begin{tabular}{|l|} 
Sanchao \\
[表紙]三抄
\end{tabular} & $\begin{array}{l}\text { Shaochao } \\
\text { [表紙]小抄 }\end{array}$ & $\begin{array}{l}\text { Jiangyang } \\
\text { [諤紙]降樣 }\end{array}$ & \\
\hline Jiangnan donglu 江南東路 & \multicolumn{7}{|c|}{ 三百二十五萬五千元百張 3.255 .400 feuilles } & \\
\hline Chizhou 池州 & $\sqrt{ }$ & & & & & & & $\begin{array}{l}\text { Mille } \\
\text { feuilles }\end{array}$ \\
\hline Xuanzhou 宣州 & $\sqrt{ }$ & $\sqrt{ }$ & & & & & & \\
\hline Nankang 南㥆 & & & & $\sqrt{ }$ & V & $\sqrt{ }$ & & \\
\hline Jiangxi 江西 & & & & $\sqrt{ }$ & & $\sqrt{ }$ & & \\
\hline Shizhou 隹州 & $\sqrt{ }$ & $\sqrt{1}$ & & & & & $\sqrt{ }$ & $\begin{array}{c}\text { Mille } \\
\text { feuilles }\end{array}$ \\
\hline Jiangxi nanlu 江南西路 & \multicolumn{7}{|c|}{ 一百二十七萬四千張 1.274 .000 feuilles } & \\
\hline Xingjun guo 興写國 & & & & $\sqrt{ }$ & $\sqrt{1}$ & $\sqrt{ }$ & & \\
\hline Hongzhou 洪州 & & & & $\checkmark$ & $\checkmark$ & $\sqrt{ }$ & & \\
\hline Xunzhou 笉州 & & & & $\checkmark$ & $\checkmark$ & $\sqrt{ }$ & & \\
\hline $\begin{array}{l}\text { Jinghu beilu Yingfu Guangxi } \\
\text { 荆湖北路應付廣西 }\end{array}$ & \multicolumn{7}{|c|}{ 五十五萬九千五百五十張 559.550 feuilles } & \\
\hline Ezhou 鄂州 & & & $\sqrt{ }$ & & & & & \\
\hline Xiazhou 峽州 & & & & & & $\sqrt{ }$ & & \\
\hline Yuezhou 嶽州 & & & & $\sqrt{ }$ & V & $\checkmark$ & & \\
\hline
\end{tabular}




\section{Entre les Yuan et les Ming : la monographie locale Huizhou fuzhi}

Dans cette deuxième section, nos sources principales sont des passages de monographies : le Huizhou fuzhi du $\mathrm{XvI}^{\mathrm{e}}$ siècle, des fragments d'une monographie plus ancienne, ainsi que deux versions d'une monographie de la province du Jiangxi. Il en résulte un récit très ancré dans le territoire, d'autant que nous n'avons pas pu identifier des textes qui nous auraient permis d'associer les témoignages des administrateurs locaux et un récit plus général de l'administration impériale, comme cela a été possible pour les Song. Restant dans le cadre de notre exposé, les théories empruntées à d'autres auteurs ne sont pas non plus exhaustives, même si le sud de l'Anhui sous les Yuan semble représentatif d'un système qui se veut très étatique dans un premier temps, mais qui se relâche dans un second.

Ces monographies présentent une caractéristique majeure : les auteurs des Ming ou bien leurs proches prédécesseurs semblent avoir un rapport plus étroit à leur territoire et aux savoir-faires locaux qu'auparavant. Les techniques dont Luo Yuan a transmis le témoignage, pour le papier épais et en grand format, avaient déjà été traitées dans les notes de Su Yijian. Autrement dit, elles étaient déjà inscrites dans un discours littéraire à propos de ce «trésor » du lettré qu'est le papier, même si les chapitres précédents du Xin 'an zhi servent à élucider le rôle du papier en tant que produit d'une économie locale et en tant qu'instrument de la fiscalité. La technique qui est narrée, pour la période Yuan, dans le Huizhou fuzhi ne se trouve pas dans d'autres recueils littéraires. On peut donc imaginer, faute d'autre trace, le travail que les enquêteurs menèrent auprès des manufactures de papier, dans le cadre de la rédaction des monographies.

\section{Des cuves à papier selon le Xin'an fuzhi et le Jiangxi tongzhi}

Sous la dynastie des Ming, les compilations de lettrés sur les objets de collection où l'on parle du papier sont nombreuses. Leur contenu est assez répétitif : dans le Zunsheng bajian 遵生八茂 de Gao Lian 高濂 (actif pendant les années 1580-1600) et dans le Kaopan yushi 考槃餘事 de Tu Long 屠隆 (1543-1605), des références sont faites à quelques papiers de Xin'an, mais rien qui permette de comprendre sa production et sa distribution sur le territoire régional ${ }^{86}$.

Nous conservons également deux monographies de la préfecture de Hui pour cette même période : l'une de 1502 ( $15^{\mathrm{e}}$ année Hongzhi), ayant pour réviseur principal Peng Ze 彭澤 et pour rédacteur principal Wang Shunmin 汪舜民, l'autre de $1566\left(45^{\mathrm{e}}\right.$ année de l'ère Jiajing), dont les coresponsables sont He Dongxu 何東序 et Wang Shangning 汪尚寧 87. Dans les deux versions, le nom du titre n'est plus Xin'an, mais désormais celui de «Monographie de la préfecture de Hui » (Huizhou fuzhi 徽州府志), selon un choix fait par Peng Ze. Le laps de temps entre la publication des deux éditions est " canonique ",

86. Gao Lian, Zunsheng bajian, Taipei, Shangwu yinshuguan, [1979?], ch. 15, p. 35r ; Gao Lian explique notamment qu'à Xin'an, on faisait du papier pour sūtra à la façon des Song. Sur Tu Long, voir plus haut, note 22 et plus bas, notes 93 et 100 .

87. Sur ces monographies voir Zhai Tunjian, «Étude comparative des monographies de la préfecture de Hui et du district de She », dans M. Bussotti et J.-P. Drège (éd.), Imprimer sans profit, Paris-Genève, EPHE-Droz, 2015, p. 289, 293, 295 et suiv. Il va de soi que ces monographies ne sont pas le fruit de travaux de deux seules personnes. D'ailleurs, dans les deux cas, on trouve de nombreux personnages cités dans les textes introductifs, même si les différentes fonctions des uns et des autres ne sont pas encore explicitées dans les pages d'ouverture des compilations. Ceci sera le cas sous Kangxi ; entre les deux éditions des Ming, il y a cependant une évolution, car le nombre de personnes impliquées augmente et quelques appellations de fonctions apparaissent ; cf. Zhai, ibid., p. 301-302. 
environ 60 ans. En revanche, il faudra attendre plus d'un siècle avant qu'une nouvelle édition ne soit publiée pendant le règne de Kangxi (1662-1723) des Qing.

Antérieurement aux deux éditions qui nous sont parvenues, d'autres, qui n'ont pas été préservées, ont été produites sous les Ming : parmi celles-ci, la Monographie de la préfecture de Xin'an (Xin'an fuzhi 新安府志) compilée sous l'autorité de Zhu Tong 朱同. Zhu Tong, pendant l'ère Hongwu (1368-1399), suivit les instructions de l'empereur de la nouvelle dynastie, qui souhaitait la compilation des monographies locales ${ }^{88}$. Cette œuvre ne fit pas l'unanimité, notamment auprès des membres des lignages qui considéraient que leur histoire n'y était pas suffisamment représentée ${ }^{89}$, mais elle fut toutefois incluse dans le Yongle dadian 永樂大典, la fameuse encyclopédie manuscrite des Ming, jadis perdue et aujoud'hui en partie reconstituée.

Récemment, Pu Xia 浦霞 a identifié et publié quelques passages de cet ouvrage inclus dans le Yongle dadian : selon lui, ces citations présenteraient la situation à Huizhou au début des Ming - mais, au moins pour la première d'entre elles, nous pensons qu'elle pourrait se référer à la dynastie des Yuan, une période à propos de laquelle le Xin'an fuzhi, compilé au début des Ming, devait rapporter de nombreuses informations. Ces brèves citations du Xin'an fuzhi dans le Yongle dadian sont les suivantes:

- Quantité de papier produit dans le district de Xiuning.

À Shuinan 水南 près [de la ville] du district lui même, ainsi que dans les trois cantons (xiang 鄉) de Yurui 虞获, Hemu 和睦, Liang'an 良安 ${ }^{90}$, il y a des foyers chargés des cuves (caohu 槽戶) qui fabriquent du papier. Sous ce règne, en plus de la production apportée en tribut mensuel de 3800 feuilles de papier bangzhi 榜紙, il y a du [papier] hemai 和買 (d'achats publics à prix harmonisé ${ }^{91}$ ), sans montant fixé.

- Le papier d'achat public à prix harmonisé (hemai zhi 和買纸).

Les arrondissements ( $d u$ 都, unité territoriale au-dessous du district) 10 et 11 du district de Jixi comptent des foyers chargés des cuves. Selon l'ancienne monographie, sous ce règne, en plus de la production apportée en tribut chaque mois de 1000 feuilles de papier bangzhi, chaque année il y a des achats publics à prix harmonisé (hemai) et à chaque fois l'évaluation de la qualité et de la quantité varie.

88. Sous les Ming, l'administration locale fut sollicitée à plusieurs reprises pour rédiger des monographies : pendant les règnes de Taizu (Hongwu $9^{\circ}$ année et $11^{\circ}$ année, 1376 et 1378 ) et de Yongle (1403-1424) ; cf. M. Bussotti, «Compiler, éditer, illustrer : les monographies du district de Xiuning », BEFEO, 95-96, 2008-2010 [2012], p. 220-221.

89. Voir le cas de la famille Jin, étudié par A Feng 阿風 et Zhang Guowang 張國旺, « Ming Longqing ben Xiuning Danxi Jinshi zupu suoshou Song Yuan Ming gongwenshu kaoxi 》 明龍拯本修掌《璫溪金 氏族譜》所收宋元明公文書考析, texte inédit transmis par l'auteur que je remercie ici.

90. Pour les trois noms de lieu Yurui, Hemu et Liang'an, voir plus bas, p. 297. Quant à Shuinan, littéralement 《au Sud du fleuve» du district (本縣之水南), il ne s'agit pas d'un xiang ni d'un du. Cependant, sur d'anciennes cartes du district, on le trouve au Sud-Est de Xiuning ; ce lieu est en correspondance avec la localité de Zhongtang 鍾塘 et avec des endroits encore nommés actuellement Xiashuinan (下水南, carte de 2005, Xiuning xian xingzheng qu huatu 休密縣行政區劃圖) ou Zhongshuinan (中水南, carte de 1988, Huangshan shi ditu 黃山市地圖). Cela ferait penser à une localité proche de la capitale du district même, autrement dit, cette cuve pourrait être à proximité de la ville, les autres cuves étant dispersées dans les campagnes environnantes. Shuinan est donc un nom de lieu, mais pas d'un quatrième xiang, contrairement à ce qui est dit parfois (voir l'article de Chen Rui, cité note 117).

91. Sous les Yuan, l'expression hemai désignerait plutôt des achats ou des confiscations réglés sur la base d'un prix fixé par l'autorité. Ainsi, le terme est traduit par « achat public à prix harmonisés » ou « achats par accords »: ces achats auraient été profitables aux autorités mongoles qui fixaient les prix; Paul Ratchnevsky et al., Un code des Yuan, Paris, PUF, 1972-1977, vol. 1 p. 229 et suiv. 
À propos du papier produit dans le district de She, chacun des arrondissements ( $d u$ ) $4,5,17$, de 30 jusqu'à 34 et 36 , a des cuves. En plus des 4800 feuilles apportées en tribut chaque mois, il y a un surplus chaque année de [papier] hemai (d'achats publics à prix harmonisé) de toute sorte; selon les moments, les règles établies pour les qualités et les quantités varient... ${ }^{92}$

Dans ces citations, nous trouvons deux nouvelles appellations désignant des types de papier. Le premier terme, bangzhi, désigne un papier pour écrits et placards (bang 榜) qui est souvent décrit comme un produit à base de bambou ${ }^{93}$. Le deuxième terme, hemai zhi, fait référence à une utilisation et, plus largement, à une modalité d'achat : il pouvait être constitué de différentes qualités de papier. Quant aux noms de lieu cités dans l'ouvrage du XIV ${ }^{\mathrm{e}}$ siècle, nous allons en reparler, mais constatons déjà que Xiuning vient rejoindre She et Jixi : ces trois districts producteurs forment une zone allongée au centre de l'ancienne préfecture qui correspond aux cours d'eau principaux, vers l'Est et en direction de la province du Zhejiang. Dans ces quelques phrases du Xin'an fuzhi selon le Yongle dadian, un nouveau terme technique apparaît aussi : l'expression à deux caractères caohu 槽戶 (foyers chargés des cuves) ${ }^{94}$, rarement utilisée ; comme nous le verrons, elle est maintenue dans le Huizhou fuzhi de 1502 pour décrire le système de production de papier sous les Yuan.

Auparavant, dans la compilation Zhongzhengde wenji 忠正德文集 de Zhao Ding 趙鼎 (1085-1147), il avait été question de foyers chargés de fours (yaohu 窑戶), occupés à produire de grandes quantités de papier à Hongzhou 洪州 dans les années antérieures à 1130 (quatrième année de l'ère Jianyan), mais par la suite, ces gens furent attaqués et exterminés et il n'y eut plus de foyers chargés du papier ( $z h i h u$ 紙户). Dans cet ouvrage des Song du Sud, l'expression faisant référence aux fours pourrait s'expliquer par le fait que, dans la préparation de la pâte à papier, on recourait à la cuisson ou à la vapeur, les fours n'ayant probablement pas d'usage exclusif ${ }^{\prime 5}$.

Beaucoup plus tard, dans le Jiangxi tongzhi 汇西通志, la monographie de la province du Jiangxi du XIX ${ }^{\mathrm{e}}$ siècle qui reprend des sources de la fin des Ming, un terme proche est employé : caofang 槽房 (« maison» des cuves). Selon les histoires officielles, celui-ci indiquerait les distilleries pendant la dynastie des Yuan ${ }^{96}$. En revanche, dans le Jiangxi tongzhi, le terme caofang sert à indiquer les « ateliers » de production du papier. Le

92. Cf. Pu Xia 蒲霞, «Zhu Tong Xin'an zhi de jiazhi ji qi liyong 》朱同《新安心》的傮值及其利 用, Huixue 6, 2010, p. 211-219, notamment p. 216 . Voir aussi les textes inclus dans le Yongle dadian shiqi juan: Haiwai xin faxian 永祡人典十七卷：海外新發現, Shanghai, Shanghai cishu chubanshe, 2003, p. 267-268. L'exemple de Xiuning est répété deux fois mais, la seconde fois, le quota de feuilles n'est que de 1800 au lieu de 3800 .

93. Ce serait un papier utilisable comme support (Schmitt, 2011, p. 53) cité d'abord par Cao Zhao puis par Tu Long.

94. Normalement, le terme caohu ne signifie pas « papetier », mais cao 槽 désigne une cuve, un canal ou une citerne. D'où l'idée qu'il fasse référence à la cuve du papetier ou à des lieux où l'cau est abondante et où l'on pouvait la stocker et éventuellement la chauffer pour certaines phases de la production.

95. Voir le chapitre 3 (p. 6) de l'ouvrage dans le SKQS en ligne ou bien les passages cités par Wei Huaxian, p. 141, 144.

96. D. M. Farquhar, The Government of China under Mongolian Rule, A Reference Guide, Stuttgart, Franz Steiner, Verlag GmbH, 1990, p. 181. Selon cet ouvrage, les caofang, contrôlés par la surintendance des alcools et produits distillés, étaient au nombre d'une centaine au début du XIVe siècle. Il y avait aussi une manufacture de papier (chaozhi fang 抄紙坊/房, vers 1260) pour les papiers monétaires et les certificats gagés. 
sujet est traité dans la section relative aux produits de la préfecture de Guangxin 廣信. Cette préfecture fut instituée sous les Yuan et elle exista jusqu'aux Qing ; son territoire correspondait à l'actuel Xinzhou 信州, dépendant de la municipalité de Shangrao 上饒. L'endroit est situé à une centaine de kilomètres au sud-ouest de Kaihua 開化, autre grand centre de production de papier pendant les dernières dynasties ; il est encore moins éloigné de Wuyuan, le district situé dans le Sud-Ouest de la préfecture de Huizhou à l'époque et qui fait à présent partie de la province du Jiangxi. Le Jiangxi tongzhi décrit les conditions naturelles qui déterminaient si certains lieux étaient « favorables à un cao 槽 : en remontant aux sources, parmi les rochers et les ravins, près des cours d'eau limpide et des courants impétueux ». Selon ce texte, la matière première est blanchie - d'un blanc immaculé, et la pâte est cuite à la vapeur puis pilée finement ; on prépare alors les " médicaments " que l'on dissout pour purifier (la pâte) et on drague l'eau à l'aide un treillis pour fabriquer une feuille. Les cuves ne se situent pas à un seul endroit et effectivement, plusieurs noms de lieu sont transcrits dans le passage qui précède la description. Le Jiangxi tongzhi conclut que « (le procédé) est subordonné à des changements continus, jamais on ne peut le connaître à fond ; d'autant plus que cela se transmet dans les demeures du peuple et les notes (à son propos) sont approximatives ${ }^{97}$. "

Le terme cao (cuve) apparaît aussi dans les textes [administratifs] à propos des cuves utilisées pour faire des alcools fermentés. Or, on ne peut pas exclure que ces cuves eussent, initialement, d'autres utilisations que la papeterie, comme ce fut d'ailleurs le cas au moment des premières productions de papier en Europe. Les moulins étaient employés pendant les mois de l'année où il n'y avait plus rien d'autre à moudre que des chiffons. En Occident ${ }^{98}$ comme en Orient, ce sont les rivières dans les vallées qui fournissent les endroits nécessaires pour développer cette production, exactement comme le présentent ces quelques lignes du Jiangxi tongzhi.

\section{Le Huizhou fuzhi et les qualités de papier des Yuan et des Ming}

Les informations tirées du Xin'an fuzhi du tout début de la dynastie des Ming transmises par le Yongle dadian sont reprises, à quelques mots près, dans les deux éditions du Huizhou fuzhi du $\mathrm{XVI}^{\mathrm{e}}$ siècle qui ont été conservées. On comprend ainsi qu'à propos des quotas, les indications transmises à ce moment-là correspondraient donc plutôt à celles du tout début de la dynastie Ming (1368-1644), à moins de les penser stables deux siècles durant ${ }^{99}$. En ouverture de la section des « marchandises » (huowu 貨物) du Huizhou fuzhi de 1502 les noms de papier, maiguang, baihua, bingyi et ningshuang, sont à nouveau cités ainsi que la description du papier fait à Longxu et de celui qui bénéficie du froid de l'hiver. L'auteur ajoute que, " sous les Song, parmi les papiers célèbres il y avait aussi ceux appelés jinzha 進简, dianzha 殿答, yuban 达版, guanyin 觀它, jinglian 京策 ${ }^{100}$ et tangzha 堂答i 》, ainsi que ceux provenant de trois cantons de Xiuning, que l'on peut voir dans la «Monographie des faits omis [par les auteurs d'autrefois] » (Shiyizhi 拾遺志). Ce

97. Jiangxi tongzhi, ch. 27. La monographie Qing, de Xie Min 謝旻, est disponible en ligne dans le SKQS ; voir plus bas, note 111 .

98. Érik Orsenna, 2013 , p. 57 et 60.

99. Voir HZFZ-HZ, juan 2, p. 55r et HZFZ-JJ, juan 7, p. 48. Dans les extraits du Yongle dadian sont donnés exactement les mêmes quotas de papier bang pour chaque localité (4800 pour She ; 3800 pour Xiuning ; 1000 pour Jixi). Pour les autres papiers, il est expliqué que les quotas et qualités ne sont pas fixés.

100. Tu Long utilise quatre caractères pour un même type de papier : le Guanyin lianzhi, cf. Zhimo biyan jian, op. cit., p. $1 \mathrm{v}$. 
bref passage s'achève par l'affirmation que la production locale était en train de décliner tandis que deux centres proches, au Zhejiang (Carte 3) ${ }^{101}$, se développaient : «Bien que l'on ait consigné cela par écrit selon l'ancienne monographie, parmi les qualités de papier de Xin'an de nos jours, il n'y en a pas d'excellente et seules celles dont on fait commerce dans les deux districts de Kaihua et Changshan 常山 sont bonnes ${ }^{102}$. ”

Dans la section du Huizhou fuzhi sur les «produits locaux offerts [annuellement] en tribut " (tugong 土貢), les informations sont présentées dynastie par dynastie : on y retrouve les données des époques Tang et Song ${ }^{103}$ qui ont fait l'objet d'explications dans la section précédente. Cette énumération chronologique, où le papier est traité avec les autres tributs, comprend des informations à propos de la dynastie des Yuan qui sont particulièrement riches par rapport aux sources habituelles. De plus, comparées à l'ancienne monographie, ces notes sont mieux organisées dans l'énumération et plus précises dans la description : les papiers sont identifiés par type ou selon leur usage et ces deux catégories sont mieux distinguées que dans le Xin'an zhi des Song. Certains passages restent cependant assez obscurs. La publication du Huizhou fuzhi de l'ère Hongzhi, à la différence de celui de l'ère Jiajing, connut une réalisation compliquée et l'édition fut critiquée par ses contemporains : l'un des reproches faits à cette monographie fut que son texte comportait de nombreuses fautes ${ }^{104}$. Mais, au moins pour la description des tributs en papier sous les Yuan, le texte de 1502 est repris à l'identique en 1566. Il s'ouvre ainsi :

Chaque année, parmi [les papiers] offerts à l'administration, il y avait du papier destiné à être envoyé au Nord (fubeizhi 赴北紙), du papier pour le Censorat provincial (Xingtaizhi 行臺紙) et du papier pour la Commission de surveillance de la circonscription locale (bendao lianfangsi zhi 本道廉訪司紙) ${ }^{105}$. Ces papiers étaient de trois sortes : le jiazhi 夾紙, le xianzhi 線紙 et le jianzhi 檢紙. Le [volume du] jiazhi envoyé au Nord, chaque année, atteignait 3000000 de feuilles. En 1313 (deuxième année Huangqing) on a envoyé une lettre officielle [à propos des quotas] afin que [le papier] ait une place parmi les matériaux du Magasin des pièces d'orfèvrerie et des joyaux (Jinyufu 金玉府) ${ }^{106} \ldots$

Notre témoignage concerne donc l'année 1313, quand apparemment - toujours selon ce texte, dans sa partie conclusive qui est discutée plus bas - fut établie une taxe d'été qui comprenait l'envoi d'un tribut de papier à la capitale. Pendant la période finale de la

101. Nous restons ici dans la même zone, dans la partie du Zhejiang limitrophe à l'Anhui et au Jiangxi. Ces papiers de qualité sont souvent cités dans les sources des Ming et des Qing.

102. HZFZ-HZ, ch. 2, p. 50v.

103. Comme expliqué plus haut (note 63), quelques caractères ont été changés dans le texte concernant les papiers des Song (voir jinglian 連: HZFZ-HZ, ch. 2, p. 52r).

104. Zhai Tunjian, 2015, p. 297-298. L'édition suivante ne fut pas si critiquée. Elle reprend ces textes tels quels sans corrections ou commentaires qui auraient pu aider à leur compréhension.

105. Le «Xingtai » ou 《Xing yushi tai » 行[御史]臺. peut être le Censorat qui contrôle la région du Jiangnan et le Liangfangsi ou [Suzheng] liangfangsi [肅政] 廉訪司, voir C. Hucker, A Dictionary of Official titles in Imperial China, Taipei, SMC Publishing INC., 1985, p. 246, 247, 312. Une première hiérarchie est donc formulée, entre les produits envoyés au Nord, donc aussi à la capitale, les papiers destinés au Censorat et finalement ceux pour l'administration de la circonscription locale.

106. Il s'agirait de l'un des bureaux de la Manufacture impériale (Jiangzuo yuan 將作院) qui contrôlait les activités artisanales; voir ibid. p. 169 ; les ateliers de fabrication portaient aussi le nom de Jinyu ju 金无局. 
domination mongole, les envois vers le Nord sont d'ailleurs importants ; les grains, surtout, sont acheminés par le canal enfin achevé, ou par voie maritime. Assez logiquement, dans les lignes suivantes de ce texte, nous lisons des informations très précises pour les qualités de papier « envoyées au Nord », que l'on peut résumer dans le tableau ci-dessous :

Tableau 3 : Différentes qualités du papier envoyé en tribut sous les Yuan, selon les données de la Monographie de préfecture de Hui du Xvi $l^{\mathfrak{e}}$ siècle

\begin{tabular}{|c|c|c|c|c|}
\hline \multirow{2}{*}{$\begin{array}{l}\text { Qualité } \\
\text { jiazhi }\end{array}$} & \multicolumn{2}{|c|}{ Dimensions de chaque feuille* } & \multirow{2}{*}{$\begin{array}{l}\text { Poids de } 1000 \\
\text { feuilles de papier } \\
50 \mathrm{jin}\end{array}$} & \multirow{2}{*}{$\begin{array}{l}\text { Quantité de } \\
\text { «bois » utilisé } \\
\text { pour } 1000 \\
\text { feuilles } \\
150 \text { jin } * *\end{array}$} \\
\hline & $2,4 \times 2$ chi & $74,88 \times 62,4 \mathrm{~cm}$ & & \\
\hline xianzhi & $2,2 \times 1,8 \mathrm{chi}$ & $\begin{array}{l}68,64 \mathrm{x} \\
56,16 \mathrm{~cm}\end{array}$ & 32 jin 8 liang & $\begin{array}{l}90 \text { jin et } \\
3,48 \text { liang }\end{array}$ \\
\hline jianzhi & $2 \times 1,65 \mathrm{chi}$ & $62,4 \times 51,4 \mathrm{~cm}$ & $20 \mathrm{jin}$ & $\begin{array}{l}55 \text { jin et } \\
4,1 \text { liang }\end{array}$ \\
\hline
\end{tabular}

"Équivalence fondée sur l'estimation moyenne d'un chi à $31,2 \mathrm{~cm}$ pendant les dynasties Song et Yuan dans le Hanyu daci dian.

** Le jin 觔 équivaut à seize liang ou onces. On peut imaginer combien étaient importantes les quantités de mûrier nécessaires pour obtenir les trois millions de feuilles, si l'on songe qu'une toute petite partie de la plante est exploitable.

Il est difficile de dire de quels papiers il s'agissait exactement, leurs noms nous aidant peu : il s'agirait d'un « papier cartonné » (jiazhi 夾紙), d'un papier « à traits » (xianzhi 線紙) et d'un papier «à lettres » (jianzhi 檢紙). Pour le xianzhi, en admettant que papier « à traits » soit la bonne traduction, il faudrait se demander si les traits pourraient avoir été tracés ${ }^{107}$ ou si l'expression serait une allusion aux traces des vergeures de la forme laissées sur la feuille; de nos jours, ce terme désigne parfois un papier à la surface un peu crépue, à la différence d'un papier de type jian à la surface uniforme. L'Histoire des Yuan (Yuanshi 元史) décrit l'utilisation pour les correspondances militaires d'un papier jian propre et enveloppé, au-dessus duquel on mettait un épais papier jia et des bandes de papier pour sceller ${ }^{108}$.

Ces papiers sont présentés du plus épais au plus fin, du plus grand au plus petit. En confrontant les dimensions et les poids, il est clair que les formats étaient importants, mais aussi que l'écart des dimensions n'était pas très grand. En revanche, l'épaisseur et la quantité de la pâte - donc le « grammage »- étaient aussi réglées, car ces éléments constituaient des gages de qualité et de résistance. Quant à la matière première, il s'agit d'une pâte de mûrier très blanche (白淨楮, littéralement « mûrier à papier d'un blanc

107. De nos jours, xianzhi est une appellation utilisée pour du papier à musique ; il pourrait alors s'agir d'une sortc de quadrillé : par exemple, il existe un papier à colonnes tracées par impression des traits de couleur bleue utilisés dans certains manuscrits des Ming.

108. Yuanshi (SKQS), ch. 101. 
limpide » ou « mûrier à papier de pure écorce ${ }^{109}$ et blanc ») et plus exactement - nous le verrons plus bas - d'une pâte traitée afin d'obtenir le degré de blancheur voulu. La rentabilité serait d'un peu plus d'un à trois, si l'on compare les poids des feuilles obtenues et de la matière travaillée.

Sur cette dernière, il devait exister toute une économie, liée à l'arbre lui-même. Car, comme le dit le Nongshu 宸書, le mûrier à papier était source de profit justement parce qu'il permettait de faire du papier. L'exemple de ce traité agricole est plutôt significatif, car Wang Zhen 王禎 (1271-1333) fut en service à Jingde, donc dans le district juste au nord-est de Huizhou (Cartes 1 et 3), avant de se rendre à Yongfeng 永豐 dans la province du Jiangxi. C'est là qu'il complète l'écriture du Nongshu et il explique qu'avec trente $m u$ 畧 de terre, on pouvait assurer une rentabilité productive du mûrier à papier sur un cycle de trois ans, en découpant dix $m u$ à l'année, puisque trois ans étaient nécessaires pour obtenir une écorce suffisamment épaisse pour être travaillée ${ }^{110}$.

Le texte du Huizhou fuzhi continue en expliquant que pour les papiers du Censorat provincial (Xingtai zhi) et de la Commission de surveillance (Lianfangsi zhi), on évaluait le total des quotas annuels, sans le calculer exactement, à 200000 feuilles. Les qualités n'étaient pas hiérarchisées en rang et les poids n'étaient pas gradués non plus; il y avait encore, pour tous les yamen 衙門 (sièges dc l'administration de la circonscription), le papier pour achat au prix harmonisé, le papier standard à usage quotidien (changke rizhi 常課日紙) et le papier pour les ouvrages classiques par achat au prix harmonisé (Hemai jingwen zhi 和買經文紙), avec des transactions calculées en millions de feuilles, sans établir de chiffres constants.

Suit enfin la description de la technique de production du papier d'écorce, qui devait être employée localement. Ce passage est intéressant car il est rare dans son genre : les méthodes connues par les textes d'époque sont limitées. Cette description serait plus ancienne, précédant les autres d'environ un siècle ${ }^{111}$. L'un des autres textes Ming sur les techniques de production de papier est, une fois de plus, un extrait d'une monographie du Jiangxi. On y lit que dans le Nord de cette province, à la fin du $\mathrm{Xvl}^{\mathrm{e}}$ siècle, les marchands de Huizhou et d'Anji 安吉 (Zhejiang) géraient les produits importés nécessaires à la pro-

109. Plutôt qu'à l'écorce, il faudrait penser à l'aubier, juste au-dessous de l'écorce extérieure ; pour ces papiers, de nos jours, on utilise aussi le terme bizhi 皮紙, «papiers d'écorce ». Dans son ouvrage Marco Polo Was in China (2013), Hans Ulrich Vogel explique que sous les Yuan on utilisa du mûrier, du mûrier à papier et notamment de l'aubier pour faire le papier destiné à monnaie, selon les sources occidentales, p. 107, 126-127.

110. Wang Zhen, Nongshu, ch. 10, guzhu (款楮, 未滿三年者皮薄不任用); voir le SKQS. À propos de Jingde, voir note 18 .

111. Le texte de la production du papier de bambou, vraisemblablement employé dans la province du Fujian, est inclus dans le Tiangong kaiwu 天工開物 (1637) de Song Yingxing 宋應星 (1587-1666), cf. «Zhongguo gudai keji tulu congbian chuji »中國古代科技圖錄荍編初集, Beijing, Zhonghua shuju, 1959, juanzhong, p. 70-77. Un autre texte provient de la monographie de la province du Jiangxi, cf. Wang Zongmu 王宗沐, Jiangxi sheng dazhi 江西省大志 de 1556. Il est repris dans d'autres versions de la monographie et dans le Liu Yueyun 劉䲞雲, Gewu zhongfa 格物中法, juan 6 inclus dans «Zhongguo kexue jishu dianji tonghui »中國科學技術典籍通菓 zonghe juan 綜合卷, Zhengzhou, Henan jiaoyu chubanshe, 1995, vol. 7, p. 1164 . En français, le résumé des deux processus est proposé dans l'ouvrage de C. Schmitt (2011, p. 36-37) qui les cite en exemple pour les deux procédés de fabrication du papier de bambou et du papier d'écorce. Pan Jixing avait consacré quelques pages de sa monographie à ces textes (1998, p. 245-248). On y apprend que la partie sur le papier apparaît dans la révision de la monographie de 1597, avec des ajouts de Lu Wanhai 陸萬垓 (1515-1600). Elle est ensuite reprise pour le Jiangxi tongzhi, cité plus haut, note 97. 
duction de papier, et que les treillis (lian 策) pour la forme à plonger dans la cuve étaient notamment produits à Huizhou ${ }^{112}$. Selon une version tardive de cette monographie, reprise sous les Qing et incluse dans le Siku quanshu, établie sur la base de sources de la période Ming, il serait difficile de prouver que la production locale du papier a pu commencer au Jiangxi avant l'ère Hongwu (1368-1399) et, plus probablement, l'ère Jiajing (1522$\left.{ }^{1567}\right)^{113}$. Au Jiangxi, l'activité papetière se serait donc développée plus tard que dans le sud de l'Anhui : vu l'importance des marchands de Huizhou dans la commercialisation des objets utilisés pour cette manufacture, on pourrait imaginer qu'ils aient aussi joué le rôle de « passeurs » à la technique de production elle-même, entre les deux provinces voisines, d'autant plus que, dans les deux localités, on produisait du "papier d'écorce ».

\section{Techniques et production du papier}

Si l'on respecte la présentation de la monographie de Huizhou de 1502, qui attribue ces données à la période Yuan, on comprend que Wang Shunmin ne serait qu'un narrateur tardif de cette technique de production utilisée deux siècles plus tôt. À propos des matières employées, le lecteur remarquera dans cette description qu'il est question d'un mûrier qui au début est «noir » et sauvage (huangheichu 荒黑楮). De l'extrait d'actinidia chinensis ${ }^{114}$ servait, comme les mucilages encore utilisés de nos jours dans la production orientale du papier, à augmenter la viscosité de la pâte et la cohésion des fibres entre elles. Par conséquent, l'adhésion avec d'autres surfaces diminue, par exemple l'adhésion de chaque feuille à la forme, aux autres feuilles, aux surfaces de séchage, et la substance facilite le détachement de la feuille sans rupture ${ }^{115}$, d'où probablement son nom de huashui 滑水 (《eau glissante ») dans le passage ci-dessous.

\section{Méthode pour fabriquer le papier (zao zhi zhi fa 造紙之法)}

L'écorce de mûrier noir sauvage est collectée, découpée en dix ou, d'une façon plus grossière, en six. Elle est mise à tremper dans un cours d'eau pure, puis sous un « couvercle » de cendres, elle est exposée au soleil. On continue à asperger [d'eau], jusqu'à ce que l'on obtienne le degré de blancheur désiré. Dépurée des cendres, dans un grand chaudron, elle est cuite jusqu'à être réduite en une bouillie tendre. On la plonge à nouveau dans l'eau peu profonde, où elle trempe pendant une journée, pour trier et éliminer les impuretés. Ainsi, ensuite, on met (la pâte) dans un récipient pour la piler le plus finement possible. Puis, elle est placée dans un sac de coton immergé dans des eaux profondes ; là, le sac est remué à l'aide d'un treuil. Une fois bien nettoyée, la pâte est versée dans une cuve. De plus, on a pris des [branches] de kiwi finement pilées ; dans

112. Pan Jixing, 1998, p. 245-246.

113. Jiangxi tongzhi, ch. 17, SKQS version en ligne, voir note 97.

114. Pan Jixing (1998, p. 214-215) traite de cet exemple parmi la «pharmacopée du papier » (yaozhi 䒛紙 ou zhiyao 紙樂) employée dans les processus de production. Il revient ailleurs sur le sujet : yangtao 楊桃, yangtao 羊桃 et houtao 猴桃 (Actinidia chinensis) sont équivalents. Signalons que yangtao 楊桃 est interchangeable avec mihoutao 㺟猴桃 (Actinidia chinensis) mais aussi avec le yangtao 陽桃 (Averrhoa carambola) selon le dictionnaire botanique de Fèvre \& Métaillé, 2005, p. 320, 529, 532. Voir aussi C. Schmitt, 2011 , p. 262 et plus haut dans cet article, note 75 . À propos de la "pharmacopée du papier », Zhang Binglun donne une longue liste recensant 39 noms de plantes, leur lieux de diffusion, la préparation du médicament, mais il n'explique pas clairement quelle était leur fonction. Voir Zhang Binglun et al., 2005, p. 29-31.

115. Le rôle des « médicaments » est de « gainer les fibres afin de leur imposer une flottaison égale qui se traduira par l'entrelacement régulier de la matière et permettra que les feuilles humides ne collent pas entre elles, là où la méthode occidentale impose d'intercaler des tapis de feutre. »; Polastron, 1999, p. 58. 
un seau d'eau, on les a imbibées et frottées : c'est ce qu'on appelle " l'eau glissante ». Une fois versée dans la cuve (cao 槽) avec de l'écorche blanchie, on agite et on bat [le tout] pour qu'il soit uniforme. On soulève la pâte avec un treillis et on obtient les feuilles qui sont alors pressées et séchées sur un mur chaud. Une à une, les feuilles sont étalées et brossées. Ensuite elles sont rognées et livrées à l'administration.

Après cette recette, nous retrouvons des informations plus administratives qui sont malheureusement très elliptiques et donc difficiles à interpréter. Il est possible que la perte de la section sur les manufactures (zaozuo 造作) dans le Zhiyuan xinge 至元新格 (Nouveaux statuts de l'ère Zhiyuan, 1264-1294) soit un frein à notre compréhension de ces sources ${ }^{116}$. Chen Rui 陳瑞, qui fait état de ce texte dans un article relativement récent sur l'artisanat sous les Yuan, n'arrive pas à esquisser la toile de fond dans laquelle ces données pourraient trouver une place plus significative et éclairante ${ }^{117}$. Malgré cela, sa théorie est que l'Anhui du Sud aurait été parmi les centres les plus importants du moment, si ce n'est le plus important, pour la production du papier de l'Empire ; le papier, mais surtout les autres trésors des lettrés et l'imprimerie xylographique auraient bénéficié localement d'une situation de gestion « mixte», entre privé et public, qui favorisa leur développement, contrairement aux cas où la gestion des produits était exclusivement gouvernementale, avec des performances plus limitées.

La partie finale du Huizhou fizhi reprend le thème de l'importance du papier destiné à la production de billets : cette monnaie, sous les Yuan, est très développée, constituant selon certains auteurs le «phénomène économique le plus significatif » de la dynastie ${ }^{118}$. Cette dernière section du texte explique d'abord que, déjà à la fin des Song, il était nécessaire de rechercher de nouveaux endroits pour la production du papier des guanzi 關子 (les « certificats » d'échange). On comprend que Huizhou fut choisie, avec un transfert de production du Sichuan à l'Anhui qui aurait donc été inverse à celui que nous avons évoqué plus haut, pour le papier des «billets » huizi un siècle plus tôt ${ }^{119}$. Car - est-il expliqué - au Sichuan on avait subi des pertes. À ce moment-là, les manufactures se

116. Cf. Paul Ratchnevsky, Un code des Yuan, Paris, Collège de France, Institut des hautes études chinoises, 1985, vol. 1, p. XII.

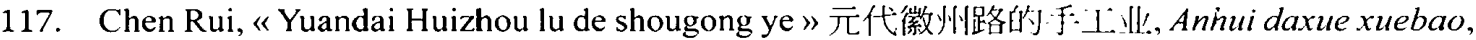
2009, 33-1, p. 101-107. Le papier est absent dans H. F. Schurmann, Economic Structure of the Yucan Dynasty, Translation of Chapter 93 and 94 of the "Yuanshi " (Cambridge - Massachusetts, Harvard University Press, 1956), où nous trouvons en revanche des informations sur les minéraux, les céréales et le riz, les tissus, le sel, les produits distillés, etc. Cette partie du texte du Huizhou fuzhi manque dans les différents ouvrages consultés en chinois à propos du papier et cela pourrait s'expliquer par les difficultés d'interprétation évoquées.

118. H. F. Schurmann, 1956, p. 131-145. Khubilai aurait veillé à mettre en place une conversion "douce " des huizi chinois vers des billets circulant déjà dans le Nord de l'empire, permettant aux Chinois de convertir leur monnaie pendant plusieurs années (Morris Rossabi, Khubilai Khan His Life and Times, London - Berkeley, University of California Press, 1988, p. 186). Dans ce passage il est cependant question de « certificats » guanzi et non de « billets » huizi. D'ailleurs, différents « certificats » auraient justement été émis à la fin des Song, entre autres pour contrer la dévaluation des « billets » : sur cc point, voir C. Lamouroux, 2003, p. 215-216, surtout la fin de la note 214 . A propos de la politique des Mongols pour développer lentement mais fermement la monnaie en papier, voir aussi $\mathrm{H}$. Ulrich Vogel, 2013, notamment p. 91 et suiv.

119. Cf. plus haut, p. 274-275. 
trouvaient dans la partie méridionale des villes, aussi les bureaux de production de guanzi furent-ils établis dans ces mêmes lieux, mais au Sichuan ils furent aussitôt détruits ${ }^{120}$.

Puis, « sous les Yuan, les foyers assignés aux cuves n'arrivaient pas à supporter (leur tâche) ; [les gens] s'enfuyaient et les migrants devinrent très nombreux. Avant la $3^{\mathrm{e}}$ année de l'ère Zhida (1310-1311), on a fait dépendre cette production des bureaux des manufactures (zazaoju 雜造局). Ensuite on a changé et elle a été soumise aux autorités constituées (yousi 有司) ». La profession aurait donc d'abord été transmise selon un système héréditaire. Ceci fait penser au cas des chauffeurs de saumure (zaohu 筐戶, zao, le four, remplaçant parfois yan 鹽, le sel) qui, sous les Yuan, travaillaient de génération en génération et possédaient les ateliers où était produit le sel et, par conséquent, des cuves et des fours ${ }^{121}$. Même si la situation des artisans de l'époque est souvent décrite comme avantageuse ${ }^{122}$, au point que le départ de certains artisans aurait été provoqué par l'administration elle-même ${ }^{123}$, selon le Huizhou fuzhi le travail des cuvistes-papetiers était pénible et ils finissaient par s'enfuir. De surcroît, ces artisans préféraient une position autonome permettant une meilleure rétribution. Le système fut donc abandonné, d'abord en assignant cette production aux manufactures qui dépendaient de l'administration du district, les zazao ju 雜造局.

Grâce à d'autres citations de la monographie de Zhu Tong incluses dans le Yongle dadian, on connaît l'existence et la localisation d'un zazao ju 雜造局 à Huizhou, qui fut détruit à la fin de Yuan, bien que le papier ne soit pas cité parmi les objets produits ${ }^{124}$. Plus tard, il est question d'institutions (yousi) compétentes : il semblerait donc qu'une institution de contrôle ait bien été établie, tandis que l'activité productive était désormais menée hors des structures administratives. Des achats de mûrier sur la base de leur véritable valeur furent également institués. Cette réforme n'eut pas le temps d'être complètement appliquée. Le texte du Huizhou fuzhi cite en effet une deuxième fois la réforme de 1313-1314 ( $2^{\mathrm{c}}$ année Huangqing) : la taxe d'été (xiashui 夏稅) comprenait des paiements commutés en nature (zhena 折納), dont des tributs de papier [pour la capitale] ; ce papier " destiné à être envoyé au Nord (fubeizhi) » semble être celui qui remplit le Magasin des pièces d'orfèvrerie et des joyaux (Jinyufiu), selon les règles que nous venons de lire en ouverture de ce texte et de résumer dans le Tableau 3.

120. Au XIII" sic̀cle, les Mongols s'installent sur les territoires septentrionaux (1215, prise de Pékin) et le Sichuan est le lieu de campagnes militaires de Mengke et Khubilai dans les années 1250 ; les désordres auxquels le texte fait allusion pourraient être liés à cette période de transition.

121. Cf. la section « Des vivres et marchandises », dans P. Ratchnevsky, Un code des Yuan, 1985, vol. 2, p. 156 et vol. 3, p. 173 .

122. En plus des compensations en salaires et biens, de l'exemption des corvées, les artisans pouvaient vendre leurs produits une fois qu'ils avaient rempli leurs quotas annuels obligatoires ; cf. H. Franke et D. C. Twitchett (dir.), The Cambridge history of China vol. 6, Alien Regimes and Border States 907-1368, New York, Cambridge Univesity Press, 1994, p. 448-449. Le contrôle des abus de la part de l'administration sur les artisans est aussi rappelé par Rossabi, 1988, p. 122.

123. Des départs furent organisés en 1280, 1290 et 1308 pour se libérer des artisans médiocres, selon Chü Ch'ing-yuan, "Gouvernement artisans of the Yuan Dynasty », in E-tu Zen et John de Francis, Chinese Social History: translations of selected studies, Washington, American council of learned socictics, 1956, p. 234-246, surtout p. 240.

124. Cf. Pu Xia, 2010, p. 217. Xic Jin 解繮 et al., Yongle dadian, juan 19781 p. 22v, dans «Siku quanshu cunmu congshu bubian », Jinan, Qilu shushe, 1997, vol. 72, p. 26. 
Par ailleurs, cette décennie fut cruciale pour l'empire mongol sur le territoire chinois, coïncidant non seulement avec la mise en place définitive du système de concours, mais aussi avec les premières rébellions qui affaiblirent les liens entre occupants et élites chinoises. Le dernier fait narré dans le Huizhou fuzhi intervient en 1319 (6 $6^{\mathbb{C}}$ année Yanyou), quand le commandant de circuit Zhu Qi 朱雼 se serait adressé au secrétariat provincial (xingsheng 行省) ${ }^{125}$ afin que dans les trois districts de She, Jixi et Xiuning, là où poussait le mûrier à papier et où était produit le papier, puisque la taxe d'été épuisait les ressources, les foyers des classes supérieures puissent récupérer par commutation le papier produit et que, selon un édit administratif, les foyers responsables des entrepôts achètent (du papier) à sa valeur effective dans le cadre d'achats à prix harmonisé. Les autorités ${ }^{126}$ craignant qu'une prescription appliquée seulement aux trois districts puisse engendrer des disparités et des pertes, élaborèrent la proposition d'appliquer des mesures similaires à l'ensemble du circuit.

Ce long texte s'achève par la description précise des lieux où la production se pratiquait sous les Yuan : "À Shexian les lieux qui produisaient le papier étaient les arrondissements ( $d u$ 都) 4, 5, 17, 30, 31, 32, 33, 34, 36, qui tous avaient des cuves. Dans les districts de Xiuning, les lieux de production du papier étaient Shuinan et les cantons de Yurui, de Hemu et de Liang'an, tous dotés de cuves. Ainsi que les arrondissements $(d u)$ 10 et 11 de Jixi. " Ce sont exactement les mêmes endroits qui sont citćs, toujours dans le Huizhou fuzhi à propos des Ming, et qui plus tôt étaient apparus dans le Xin'an fuzhi, d'où notre difficulté à déterminer une date précise pour ces données. Dans cette conclusion, pour Shexian et Jixi, on a énuméré des arrondissements $(d u)$; pour Xiuning, trois cantons (xiang 鄉) et, sur la base de monographies contemporaines, on pense que Yurui et Hemu occupaient la partie sud-occidentale du territoire, en direction de Qimen et au nord de Wuyuan ${ }^{127}$. Selon le même Huizhou fuzhi, sous les Song et les Yuan, le district de Xiuning comptait 11 cantons (xiang) et 60 village (li) : Liang'an était au nord, Hemu au sud et Yurui à l'ouest ${ }^{128}$. Malheureusement, faute de cartes d'époque assez détaillées, nous avons dû renoncer à cartographier les sites concernés ${ }^{129}$.

125. Xingsheng pour Xingzhong shusheng 行中書省, structures administratives des zones provincialcs. Selon Chen Rui, il s'agit de celle de Jiang-Zhe 江浙. Quant à Zhu Qi (1260-1321), on trouve en ligne quelques informations sur des sites génériques : surnommé Jingzhou 竟周, originaire de Xintai 新泰, il occupa son dernier poste à Huizhou où il fut nommé en 1316-1317. Dans ce texte il est présenté comme zongguan 總管, qui était normalement l'administrateur en charge d'un circuit ( $l u$ 路) sous les Yuan.

126. Le texte cite le dusheng 都省, terme qui peut désigner le département des affaires d'État ; on y trouve aussi l'expression tingta 停摆, proche de tunji 屯績, c'est-à-dire stocker un produit, éventuellement à des fins spéculatives. Pour les achats à prix harmonisé, voir plus haut note 91 .

127. Une monographie moderne de Xiuning reprend des informations de la monographie du district de la troisième année Daoguang (1823) : Hemu couvrait le territoire de deux du (19-20) avec une dizaine de villages, et Yurui comprenait $4 d u(30-33)$ et 35 villages. Cf. Xiuning xianzhi, Hefei, Anhui jiaoyu chubanshe, 1990, p. 44-45.

128. HF-HZ, juan 1, p. 51-52. Les mêmes lieux se trouvent dans le Xiuning xianzhi de l'ère Wanli, où il est expliqué que cette division était en place au début des Song (975) et que les xiang furent transformés en $d u$ pendant les Yuan ; voir Xiuning xianzhi, ch. 2 (舆地志, 治革). Je remercie A Feng pour cette information.

129. Les deux monographies de préfecture du $x \vee I^{\circledR}$ siècle n'ont pas de cartes si détaillées; on trouve des cartes des arrondissements ( $d u)$ pour Xiuning, mais c'est le seul des trois districts où, dans notre texte, les lieux sont donnés par cantons (xiang). 


\section{L'épilogue de la production dans la préfecture}

À la fin de la dynastie des Ming, dans des ouvrages édités à titre privé, tel l'atlas Guangyu kao 廣锤考 ${ }^{130}$, on cite pour Huizhou exactement les mêmes produits que dans les ouvrages historiques plus anciens, compilés par l'administration locale ou centrale de l'encre, de la laque, des pierres à encre, du papier et du Coptis chinensis, etc., mais sans aucune précision sur leurs districts de production. Dans l'atlas, le papier est aussi mentionné parmi les produits de Guichi 貴池, alias Chizhou 池州. Pour notre préfecture, on donne des noms de types de papier (baihua, bingyi et ningshuang) qui semblent attester la reprise à l'identique des sources précédentes, bien que les éditeurs aient été des gens de Huizhou ${ }^{131}$, ce qui aurait pu faire espérer quelques contributions originales.

Entre-temps, certains locaux avaient migré et fait fortune, parfois grâce au papier. Par exemple, pendant l'ère Jiajing (1522-1567), Ruan Bi 阮预 originaire de Yansi zhen 岩寺鎮, dans le district de She, émigra à Wuhu 無湖 (une localité plus au nord, sur la rive méridionale du fleuve Yangzi). Il est connu pour figurer parmi les riches marchands de Huizhou, célèbre grâce aux teintures de tissus et aux papiers teints ; sa réussite, qui lui aurait permis d'importer produits et main-d'œuvre, mais aussi d'ouvrir des activités hors de Wuhu, est rappelée dans les monographies locales plus tardives, ainsi que dans le Taihan ji 太函集, compilé par le puissant Wang Daokun 汪道昆(1525-1593) ${ }^{132}$.

Des travaux sur l'histoire locale de la dynastie des Ming ont aussi permis de repérer un document qui relate que Wang Daoguan 汪道貫 (1541-1591, le frère de Wang Daokun) et Fang Yongbin 方用涁 (1542-1608) de She, firent venir des artisans chez eux pour fabriquer des objets en papier, d'abord deux rideaux (à partir de 15 grandes feuilles de papier), ensuite un vêtement rituel ou destiné à " recouvrir » une divinité (shenyi 神衣) ${ }^{133}$. Ce qui est intéressant dans cette anecdote, en dehors de la confirmation que cette production d'objets est attestée dans une rare source de première main (des archives privées), c'est le caractère itinérant de la prestation décrite, ce qui confirme des pratiques de travail évoquées également pour d'autres artisans locaux, tout particulièrement pour certaines catégories d'imprimeurs.

Les entrées consacrées au papier vont continuer dans les monographies suivantes, alors que l'on assiste à un déclin de la production; sous les Qing, on fait état du manque de mûrier à papier et, pendant la période républicaine, de son remplacement par d'autres matières ${ }^{134}$. Pendant la deuxième moitié de la dynastie, selon des travaux récents, une

130. Le Guanyu kao 廣輿考 est une réédition du célèbre Guanyu $u$ 廣輿圖, avec des compléments ; elle fut réalisée vers 1595 et comprend des textes signés par des gens originaires de Huizhou. Cf. W. Fuchs, "The «Mongol Atlas» of China by Chu Ssu-pen and the Kuang-yii-t " $u$, with 48 fac-simile maps dating from about $1555 »$, Monumenta Serica, 1946, monograph VIII, surtout p. 24-25.

131. La préface et la post-face indiquent que les deux auteurs venaient de Xingyuan 星源, qui serait un des anciens noms de Wuyuan. Il ne s'agit donc pas du Zhejiang, comme le dit Fuchs, mais du Jiangxi.

132. Chen Qiyu 陳基余 et al. (éd.), Anhui wenhua shi 多徽文化建, Nanjing, Nanjing daxue chubanshe, 2000, vol. 2, p. 1573-1574.

133. Chen Zhichao 陳智超, Meiguo Hafu daxue Hafu yanjing tushuguan cang Mingdai Huizhou Fangshi qinyou shouzha qibai tongkao shi (美國哈佛人學哈佛㗆京圖畫館藏明代徽州方氏親友手 札七西通考釋), Hefei, Anhui daxue chubanshe, 2001, vol. 1, p. 2-3, 459, 497-498.

134. Zhang Peifang 張佩范, Liu Dakui 劉人槐, Shexian zhi 㸹縣志, 1771, juan 6, p. 5 r (p. 335 dans l'édition de «Zhongguo fangzhi congshu: Huazhong difang», Taipei, Chengwen chubanshe, 1975); Shi Guozhu 石国柱, Xu Chengyao 許毮琴, Shexian zhi, 1937, juan 3, p. 19r (p. 423 dans ibid.). Les deux sources évoquent la disparition des foyers-papetiers à She, mais la plus récente explique qu'à présent « on prend les tiges du riz (daorang 稻穰) produit dans l'Est de She ou de l'écorce de mûrier 
production de papier persista à Jixi, et plus exactement à Zhaitan 宅坦. Dans ce village, trois cuves, six mortiers hydrauliques et au moins 70 ouvriers auraient été actifs dans la production du papier pendant la période Jiaqing (1796-1821), mais les auteurs qui évoquent ces faits ne citent malheureusement pas leurs sources ${ }^{135}$. Une représentation précise de ces espaces de travail reste difficile à modéliser. Des publications (relativement) récentes ${ }^{136}$ font mention de ces mortiers, dont on connait l'existence par des ouvrages plus anciens ${ }^{137}$, mais je ne suis pas en mesure d'établir avec certitude le moment où leur utilisation se généralise dans la région qui nous concerne ${ }^{138}$.

Pour une autre localité du district de Jixi, le village de Jikeng 際阬村 au cœur du district, on explique qu'en raison de sa localisation et de sa richesse en bambou, elle fut choisie pour y créer un atelier de huozhi 火紙 (papier pour le feu) à partir de 1903. Zhou Yugui 周玉貴 y aurait installé un petit atelier à production limitée où travaillaient une dizaine d'employés et des saisonniers locaux. L'endroit se serait ensuite transformé en une véritable usine aux implantations multiples, jusqu'à son évolution vers une production de papiers variés dans les années 1930, 1950, puis de nouveau dans les années 1980, année qui correspond aussi au déménagement définitif de l'activité ${ }^{139}$. Le huozhi était un papier enduit de nitre qui servait à allumer le feu ; il est donc normal que sa production ait laissé place à d'autres types de papier dans l'Après-guerre. Cependant, le terme huozhi peut aussi désigner le papier qui est brûlé pour les défunts, et il cst lćgitimc de sc demander si la production n'a pas été d'une tout autre nature. Dans ce cas, le changement dans l'interprétation du terme aurait été introduit par les auteurs contemporains pour des

commun (sangpi 桑皮) qui vient du Sud du district. » ̀̀ ce moment, il n'y a donc ni mûrier à papier ni bambou, mais l'activité prospère.

135. Bao Yilai, 2005, p. 128.

136. Pour l'utilisation de ces outils dans la préfecture de Xuanzhou, voir la fin de cet article. Une photographie de ces « piles à broyer » reconstituées se trouve dans le récent livre de Polastron ( $L e$ trésor des lettrés, Imprimerie nationale, 2010, p. 128), mais face à ces objets persiste le doute d'une reconstitution à partir des sources écrites diverses et non à partir de pièces anciennes. Une image de ces piles se trouve dans la planche 9 (Broyer des bambous) d'un album en couleurs de la dernière dynastie conservé à la Bibliothèque de l'Institut de France, qui illustre les étapes de la fabrication du papier de bambou. Ce manuscrit (MS1066) a été donné à la Bibliothèque par Nicolas Desrmarest (1725-1815); ses planches ont été reproduites et commentées par Jean-Pierre Drège, Chine Bambou Papier, Paris, Institut de France - Imprimérie de l'Indre, 1994. Selon ce dernier, ce type de pilon qui fonctionne par un système de leviers était déjà employé avant le début de notre ère et, «à partir du X: $v^{e}$ siècle, la force hydraulique succéda à l'action de l'homme, les piles étant montées en série ». Ibid. pl. 9 verso.

137. Une représentation précise de ces espaces de travail est difficile à imaginer mais on peut se référer à deux entrées incluses dans le Sancai tuhui 三才圖繪 (ch. 10 p. 38) pour le caodui 槽碓 et le shuidui 水碓, le deuxième étant une structure plus élaborée que la première, où plusieurs marteaux sont activés par l'eau. Cf. Wang Qi 王圻 et Wang Siyi 王思義, Sancai tuhui (zhong), Shanghai, Shanghai guji chubanshe, 1988, p. 1286-1287. Voir aussi des sources plus anciennes, telles que le Nongshu, juan 19, p. 16-17, version du SKQS en ligne.

138. La question des outils n'est pas négligeable et elle mériterait des recherches ultérieures. Françoise Sabban a démontré par ses recherches sur le sucre à quel point l'utilisation du moulin à cylindres, ou mieux, de différentes sortes de moulins, est représentative de différents systèmes productifs. Voir F. Sabban, " L'industrie sucrière, le moulin à sucre et les relations sino-portugaises aux XVI ${ }^{e}-\mathrm{XVIII}^{\mathrm{e}}$ siècles ", Annales. Histoire, sciences sociales 48, 1994, p. 817-861.

139. À côté de Nancao duqiao 南曹渡橋, dans le même district. Ces informations sont reprises de l'ouvrage généraliste et collectif Huizhou wuqiancun 徽州五千村 (Hefei, Huanghan shushe, 2004), volume sur Jixi, p. 172-175, où l'on explique que l'endroit fut aussi un centre de production d'encens. 
raisons idéologiques évidentes ${ }^{140}$. Il serait intéressant de mener d'autres recherches, mais il est clair que ce produit était un papier essentiellement fait à partir de bambou : il avait peu à partager avec les anciens papiers d'écorce produit localement et décrits dans les monographies locales les plus anciennes.

\section{Monographies et sources locales à propos de l'Anhui méridional}

Dans cette dernière section, nous allons rapidement évoquer l'histoire de produits «précieux » dont les noms reviennent souvent dans la littérature consacrée au papier. Sans volonté d'en restituer une étude complète, car des monographies existent et l'argument dépasserait les limites de nos intérêts, il est pertinent d'évoquer ces types de papiers et leurs lieux de production pour compléter notre analyse et lui donner un cadre plus étendu. Faute de documents directs, ces considérations nous permettent d'esquisser la cartographie d'une région de production dans laquelle la préfecture de Hui apparaît montagneuse et très enclavée. Elle partage au sud ses confins avec les territoires de Changshan et Kaihua de la province du Zhejiang et avec l'ancienne préfecture de Guangxin dans la province du Jiangxi ; au nord s'étend une vaste région entre le fleuve Yangzi et le relief en question, région qui produit un papier encore renommé de nos jours.

En plus d'une « ouverture » spatiale, ces pages offrent aussi une perspective temporelle diffërente : pour la préfecture de Huizhou, les témoignages sur le papier s'amoindrissent avec le temps, mais ils persistent pour la région. De nouvelles formes de récits apparaissent donc à côté des monographies, comme les témoignages littéraires de la période républicaine, par exemple, qui se veulent objectifs et descriptifs d'un travail accompli par des artisans qui, de leur côté, ne sont jamais les auteurs de tels écrits. Le tout est repris, englobé et retravaillé dans un discours contemporain - que nous n'avons pas exploré, puisqu'il se fait aussi par d'autres biais que l'écrit, sans plus avoir recours au papier comme support de transmission.

\section{Les papiers de Chizhi et Xuanzhou}

Pourquoi le meilleur papier de Chine provient-il de l'Anhui, et plus précisément aujourd'hui du district de Jingxian 涇縣 où sa production s'est réfugiée? La géographie l'explique clairement : région de vallées perdues à proximité du massif vertigineux du Huangshan 藏山, elle dispose de toutes les ressources naturelles nécessaires. Une eau très pure en permanence, le combustible à profusion, les végétaux pour la fibre, et ceux pour le zhiyao ou médicaments du papier... Le xuanzhi s'est d'abord appelé huizhi, du nom de la province qui le produit, l'Anhui ; puis xuan parce que la ville qui le commercialisait était Xuanzhou (de nos jours Xuancheng) ${ }^{141}$.

Cette citation d'un ouvrage contemporain me semble à retenir pour sa simplicité, certes un peu expéditive, mais qui va à l'encontre des «pérégrinations » auxquelles nos disciplines académiques et érudites nous contraignent. Selon ce raisonnement, une continuité dans la production locale de papier de qualité se dégage à l'échelle régionale, en même temps

140. Le huozhi « papier à brûler » est décrit par Song Yingxing dans le Tiangong kaiwu 天上開物 (zhong p. $72 \mathrm{v}$ ) pour des finalités rituelles ; voir ce texte dans l'exemplaire de la fin des Ming reproduit dans "Zhongguo gudai banhua congkan ", Shanghai, Shanghai guji chubanshc, 1988, p. 950. Nous avons évoqué ce type de papier à propos des Song, voir plus haut, note 80 .

141. Polastron, 1999, p. 43-44. 
que les pôles principaux de cette manufacture semblent se déplacer, au fil des époques, d'une préfecture à l'autre, et surtout d'un versant à l'autre des massifs du Huangshan. Certains lieux que nous avons évoqués se trouvent en fait au nord de Huizhou. Ils ont changé de nom et de subdivision administrative au cours des dynasties successives : en direction du nord-ouest, près du Fleuve Bleu, se trouve Chi, ou Guizhou ; quand on quitte le Huangshan vers le nord-est, on traverse d'abord Jingxian 㽟縣 et ensuite Xuanzhou 宣州 (Xuancheng 宣城), cette région étant parfois appelée Ningguo 蜜或, du nom de l'ancienne préfecture (Cartes 3 et 4). Dans l'un des rares ouvrages encyclopédiques pour marchands qui nous soit parvenu, le Shishang leiyao 士商類要 des années 1620, nous découvrons, parmi les routes empruntées par les marchands, un parcours de 700 li entre terre et eau qui réunissait Si'an 四安 à Huizhou, transitant justement par deux zones de production du papier : Ningguo et Jixi ${ }^{142}$.

Les informations concernant une production de papier dans la préfecture de Xuan sont encore plus anciennes que les sources pour She. Zhang Yanyuan 張彣遠 (813879 ?) en fait mention ${ }^{143}$ et, dans l'Ancienne Histoire des Tang, il est écrit que pendant la première moitié du vIII ${ }^{\mathfrak{a}}$ siècle, de la macle (空青石), du papier et des pinceaux, ainsi que de la coptis japonica furent envoyés en tribut via les bateaux de la commanderie de Xuancheng ${ }^{144}$. Dans la Nouvelle Histoire des Tang, le papier est inclus dans les tributs de Xuanzhou (Xuancheng jun), de Shezhou (Xin'an jun) et de Chizhou, mais selon l'Histoire des Song, Xuancheng n'offre en tribut que des pinceaux ${ }^{145}$. Cependant, un papier dit de Xuan continue à être produit, comme le confirme le Song huiyao. Xuan fait partie, autant que Chi, She et d'autres localités, des grands centres producteurs de papier du circuit occidental du Jiangnan 江南西路 en $1077^{146}$.

Trois ans plus tôt (1074, Xining 7), «Zhang Cheng 張誠, vice-rapporteur à la Cour des Affaires militaires (樞密副都承旨), demanda “que la Commission des finances calcule d'une façon approximative les [quotas] réguliers annuels de xuanzhi", en envoyant un "officier supérieur des armées" (jun dajiang 軍大將) ou un "commissaire au palais" (dianshi 殿侍) afin que les produits soient escortés sous contrôle militaire de la préfecture à la capitale ". On explique ensuite que les papiers de format inférieur au (papier) jiang étaient faits dans le circuit de Hangzhou, à raison de 50000 unités par an. Puisque dans l'administration comme en privé, on utilisait beaucoup de papier, les dimensions

142. C'est la dix-septième route, p. 27rv du Shishang leiyao de Cheng Chunyu 程春宇: voir la copie photostatique conservée à la Bibliothèque de l'IHEC (2007). Cet ouvrage fut imprimé à Nankin, mais son préfacier est originaire de Huizhou, parmi les nombreuses routes présentées, ce qui explique que Huizhou occupe une place importante.

143. Zhang Yanyuan, Lidai minghua ji 歷代名畫記, juan 2, p. 8v, SKQS, version en ligne.

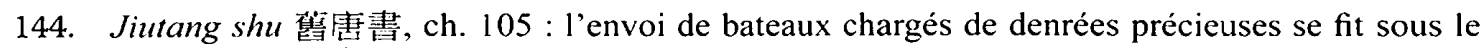
contrôle de Wei Jian (韋堅, qui serait mort en 746), aussi ces faits sont-ils relatés dans sa biographie, p. 3222-3225, et plus précisément p. 3223 .

145. Voir plus haut les références données aux notes 36 et 37 . Pour Xuanzhou, il n'y a pas non plus de tribut de papier pour l'ère Yuanfeng (1078-1085) selon le ch. 6 du YJZ, mais sont notés des tributs de 10 pièces de ramie, 30 jin de coptis japonica et 500 pinceaux (土貢白紀布一十正黄連三十斤滗 五百管). En revanche, selon la même source, Chi était tributaire de 1000 feuilles de papier, ce qui par aillcurs cst le seul tribut local inserit pour cette préfecture dans le YJZ; éd. de 1984, vol. 2, p. 244. Voir aussi plus haut, Tableau 2.

146. Xuanzhou pour la dachao lianzhi et la sanchao lianzhi, Chizhou pour la dachao lianzhi, voir plus haut, Tableau 2. 
ne devaient pas être confondues avec celles du papier de Xuan ${ }^{147}$. Le fait que le papier de Xuan aurait encore eu une place de choix au XII ${ }^{\circ}$ siècle est indirectement confirmé en 1156 , lorsque fut supprimée, en même temps que beaucoup d'autres postes, la fonction d'officier chargé de diriger le dépôt du papier de Xuan (jian Xuanzhi ku chenshi 監宣 紙庫臣使 $)^{148}$.

Le papier ne fait pas non plus partie des «produits locaux » de la section consacrée à la préfecture de Ningguo - qui inclut Xuangcheng, Nanling 南陵, Jingxian, Ningguo, Jingde et Taiping 太平 -dans l'ouvrage encyclopédique Fangyu shenglan au XIII' siècle. Zhu Mu cite parmi les principaux produits de la préfecture les fameux pinceaux faits de poils d'animaux (zihaobi 紫毫筆), des tapis et tentures (hongxiantan 紅線毛炎) et le mugua 木瓜 (cognassier [ou papayer dans les régions méridionales]). Il explique qu'au moment où la plante allait fructifier, des fleurs en papier y étaient apposées : l'exposition à l'humidité nocturne et à la chaleur du jour finissait par produire (sur les fruits ?) des motifs rouges, décoratifs et très appréciés. C'est donc dans une brève citation (à la lecture incertaine) ${ }^{149}$ que le papier est cité à propos de Xuanzhou, et ce pour une application « futile ».

Au contraire, le papier du district de Chi est cité dans les histoires dynastiques, dans le Song huiyao, ainsi que dans le Fangyu shenglan. Celui-ci parle du zhazhi 答紙 (un papier destiné à la correspondance ou à la rédaction des mémoires) et transcrit un long texte qui est, en réalité, un poème dans lequel Wang Anshi 王安石 ( $z i$ Jiefu 介甫, 10211086) remercie Wang Weizhi 王微之 qui lui a offert du papier de Chi. Sous cette forme et avec son titre, ce remerciement poétique sera reproduit beaucoup plus tard dans les monographies locales de Chizhou ${ }^{150}$.

En complément à ce poème, la Monographie locale de la préfecture de Chi de 1778 affirme que le papier provenait de Guichi 貴池, Shidai 石埭, Jiande 建得 et Tongling 銅陵. Le papier était de différentes sortes, obtenues en pilant l'écorce du mûrier à papier (chu et gu 款, Broussoneita kazinoki Sieb) et du santal (tan 檀). Sa production donnait du travail à beaucoup de monde dans la préfecture. Mais les auteurs des Qing, après avoir expliqué que le papier de Chi fut utilisé pour les tributs sous les Tang et les Song, ont eux aussi recours à la citation. Ils évoquent deux ouvrages de l'époque Song, dont le Dongbo zhilin 東坡志林: Su Shi 蘇軾(1037-1101) y fait l'éloge du papier « plaque de jade ", blanc pur de Chi et de She (Chi She jingbai yuban 池歌精白玉版), le seul

147. SHY, xingfa 二/禁約; vol. 7, p. 6512 (ce $165: 34$ ). Zhang Cheng apparaît plusieurs fois dans le Songshi mais je ne l'ai pas trouvé répertorié dans le Songren zhuanji ziliao suoyin de Chang Bide 昆彼得 et al., 1977.

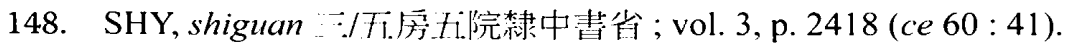

149. La citation est difficile à interpréter en raison d'un caractère écrit parfois $z u$ 鏃 (pointu comme une flèche), parfois $c u$ 簇 (groupés, réunis); le premier est dans le Fangyu shenglan (1986, ch. 15, p. 172), le deuxième dans le Bencao gangmu 本草綱目 (1578), ce qui montre que, déjà sous les Ming, on éprouvait une difficulté d'interprétation de la citation; Li Shizhen 李时珍, Bencao gangmu zhenzangban 本草綱目珍藏版, Beijing, Beijing yanshan chubanshe, 2006, p. 861. Voir aussi le Nongshu de Wang Zhen, ch. 9, mugua 木瓜, dans la version du SKQS en ligne.

150. Cf. Liu Quanzhi 劉權之et Zhang Shifan 張土範, Chizhou fuzhi 池州㑏, 43" année Qianglong (1778), fac-similé inclus dans «Zhongguo fangzhi congshu : Huazhong difang », Taipei, Chengwen chubanshe, 1985, p. 1450 : Song Wang Anshi yunchou Wang Weizhi zeng Chizhi 宋王

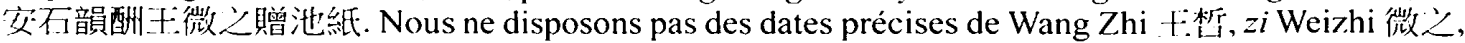
mais nous savons qu'il fut proche de Wang Anshi lorsqu'il fut en service à Chizhou; on trouve en ligne un texte l'évoquant (http://www.xz.bu.com/7/view-3800022.htm, consulté le 5/04/2014), notamment à propos des difficultés posées par son nom, qui est écrit avec trois calligraphies différentes (梠, 哲, 晢). 
papier à pouvoir valoriser complètement les qualités de l'encre. Le Shilin yanyu 石林 燕語 est également cité : Ye Mengde 葉夢得 (1077-1148) y écrit que le 《papier de chanvre blanc » (baima zhi 白麻紙) était en réalité un papier de mûrier de la préfecture de Chi ${ }^{151}$. La monographie nous livre en dernière information que le papier utilisé par le président du Département des Affaires d'État Zhou [? Hubu Zhou shangshu 戶部周 尚畫] pour ses registres (户部冊) pendant le règne de Hongwu (1368-1399) des Ming, était réalisé à Chi et plus exactement près de la Pagode Lingzhi 靈芝塔, un endroit qu'il est encore possible de repérer sur les cartes modernes de la région de Guichi dans la préfecture de Chizhou ${ }^{152}$.

Guichi est aussi mentionné pour son commerce du papier dans des documents populaires de la fin des Qing, notamment dans des généalogies imprimées en caractères mobiles dans le district de Qimen à Huizhou. C'est le cas d'une généalogie des Chen 陳 de Zhuyuan 竹源 réalisée en toute fin du XIX ${ }^{\mathfrak{e}}$ siècle : d'après le contrat, le « maître de généalogie » (pusi 譜间) se serait rendu dans cette ville pour y acheter dix mille feuilles de papier de 1,8 chi de largeur et 1,4 chi de hauteur, avec un poids (donc un grammage) et selon un prix déjà convenus avec le chef du clan ${ }^{153}$. La rédaction de la généalogie est un acte important dans l'histoire d'un lignage, ce qui suffirait à justifier ce déplacement vers un centre connu pour y acheter un papier de bonne facture. Comme il s'agissait d'une entreprise éditoriale hors commerce, ce choix n'était pas fondé sur des critères de rentabilité. Mais si les gens du clan avaient eu à leur disposition un tel papier sur place, ils n'auraient pas fait autant de route pour s'en procurer. Donc, non seulement le district de Qimen n'est jamais cité dans les textes de Huizhou sur le papier, mais cette anecdote tend à confirmer qu'à la fin de la période impériale, la production avait décliné dans la préfecture de Hui, ce qui avait contraint le maître de généalogie à s'en procurer ailleurs. Il est intéressant de constater que le maître et ses ouvriers ne se sont pas approvisionnés au Jiangxi, leur région d'origine ${ }^{154}$, qui était également un lieu de production du papier.

\section{La mythologie du papier : du papier du Chengxin tang à celui de Xuan}

Cependant, dans l'Antiquité, un papier d'une tout autre qualité que celui produit à Xuan et à Chi était beaucoup plus convoité par les lettrés : le Chengxin tang zhi 澄心堂紙 ou «papier de la Salle du cœur pur», un produit du x ${ }^{\text {e }}$ siècle. Dans les sources des Song que nous avons étudiées plus haut, son nom a été plusieurs fois cité, par exemple dans les pages du Xin'an zhi et dans les ouvrages qui reprennent cette monographie locale ${ }^{155}$. Selon certaines de ces sources, «... le papier du Chengxin tang... était un produit de premier choix, qui venait des préfectures de Chi et de She ». Ce papier « mythique » pour

151. Les citations dans le Chizhou fuzhi sont très abrégées. Les deux ouvrages sont disponibles dans le SKQS en ligne. Dongbo zhilin, juan 10, p. 7 et Shilin yanyu, juan 3, p. 8.

152. Je n'ai pas identifié ce personnage. Les registres hubu sont normalement les « registres jaunes ». "La Pagode de Lingzhi » serait un nom de lieu, correspondant au village Lingzhi zhen 鎮/ cun 村, entre Chizhou et Guizhou, dont les autorités font remonter l'existence à la dynastie des Tang : http:// www.ahage.net/chizhou/ 20940.html consulté le 05/04/2014.

153. Cf. Chen Qi 陳琪, «Qingmo Huizhou Qimen Zhuyuan Chenshi zongpu wenshu »清末徽州祁》 阴竹源陳民宗垣文書. Faguo hanxue 13, 2010, p. 332-358, surtout p. 339-340.

154. L'équipe d'artisans venait en partie de Fuzhou 撫州 (Jiangxi) et de Fuliang 浮梁, ibid., p. 337. L'autre option est que l'auteur de l'article cité à la note précédente, au moment de transcrire ces documents, ait confondu Guixi 貫溪 (au Jiangxi) et Guichi.

155. Voir plus haut, p. 281-282. 
ses qualités de blancheur et de solidité ainsi que pour son parfait rendu des traits tracés à l'encre, prend le nom de la «Salle du cœur pur» (Chengxin tang 澄心堂), le nom d'un palais à la cour des Tang du Sud (r. 937-943) à Nankin. C'est au dernier souverain, Li Yu 李煜 (r. 961-975), que certains auteurs attribuent l'invention de ce papier, ou plutôt à un artisan du Sichuan engagé à son service. Après que le royaume des Tang eut été vaincu par les Song, le Chengxin tang zhi, encore conservé dans les dépôts, fut dispersé. Ces faits nourrissent une tradition lettrée d'anecdotes et ce papier est célébré par une suite de compositions littéraires, dont des poèmes. Pan Jixing a réuni ces extraits et s'en est servi pour reconstituer la circulation de quelques rames de papier d'un personnage à un autre. D'une part Liu Chang 劉敞 (1019-1098) en aurait offert à Ouyang Xiu 歐陽修 (10071062), qui en aurait ensuite donné une partie à Mei Yaochen 梅堯臣 (1002-1060); Mei Yaochen en aurait reçu aussi de Mei Qiu 梅求 (1019-1079). Un autre échange de papier aurait eu lieu entre Mei Yaochen et Pan Gu 潘谷 ${ }^{156}$. Ce dernier, originaire du district de She, est surtout connu pour avoir été un producteur d'encre, mais il aurait profité des compétences des papetiers locaux pour faire fabriquer sur place un papier similaire à celui du Chengxin tang ${ }^{157}$.

Si nous examinons très attentivement les sources mineures et locales, nous constatons que ce papier mythique hante depuis longtemps l'esprit des savants de Huizhou. Xu Chengyao 阡承堯 (1874-1946, docteur en 1904, originaire de She) est surtout renommé pour avoir été le rédacteur principal du Shexian zhi 歌縣誌 (Monographie du district de She), achevé en 1937, après environ cinq ans de travail ${ }^{158}$. Mais il est aussi le compilateur d'autres ouvrages, parmi lesquels le Sheshi xiantan 㰸事閑談 (Bavardages sur les affaires de She), publié très récemment ${ }^{159}$. Ce recueil de trente-et-un chapitres, connu aussi sous le titre de Shegu 翖故, est apprécié car il réunit les notes que Xu aurait prises lors de la réalisation de la monographie : c'est donc une anthologie plus proche des sources de première main qu'une monographie traditionnelle, et dans cette partie de l'Anhui le Sheshi xiantan évoque un important précédent ${ }^{160}$. Au chapitre 17, il est écrit à propos des «Quatre trésors de Xin'an » :

Le Banyun manbi 扳芸漫筆 ${ }^{161}$ citant le Suhu bianlu 素蛣便錄 dit que ceux que le Shezhi appelle « les quatre trésors de Xin'an » sont : le papier du Chengxin tang, les

156. Cf. Bao Yilai, 2005, p. 100, 129-130.

157. Voir Pan Jixing, 1998, p. 178-179. Ces sources poétiques, certes intéressantes, célèbrent ce papier et nous disent combien il était précieux et cher, mais n'ajoutent rien in concreto à ce que nous venons de dire sur les lieux et les techniques de production. Elles sont reprises par Hu Huagai et Zhang Binglun (2005, p. 271-275), ainsi que Bao Yilai (2005, p. 128-133). Ces auteurs fournissent des noms de livres ou d'autres ouvrages anciens qui sont considérés comme réalisés sur papier Chengxin tang, notamment des estampages.

158. Voir Zhai, 2014, p. 293, 308, 315-320.

159. Version critique collective parue dans le «Anhui guji congshu », Hefei, Huangshan shushe, 2001. Le passage qui est cité dans cet article est tiré du vol. 1, p. 598-599.

160. Cheng Minzheng (程敏政, 1445-?), avec son Xiuning zhi et son Xin'an wenxian zhi 新安文 嘓志 (Monographie de documents de Xin’an) ; cf. M. Bussotti, « Compiler, éditer, illustrer... », 20082010 [2012], p. 213-290.

161. Comme l'explique une autre entrée du Sheshi xiantan (2001, vol. 1, p. 596, vol. 2, p. 621), le Banyun manbi est un texte de Jiang Meibin 江梅賓 ( $z i$ Shaolian 紹连 1738-1811) imprimé au début de l'ère Jiaqing ; le Suhu bianlu est un ouvrage de la période Qing, signé par son père Jiang Dengyun 江登整 et largement cité par Meibin. 
pinceaux de Wang Boli 汪伯立 (Song ?), les encres de Li Dinggui et la pierre à encre « cœur de jujube » de Longwei 龍尾 (à Wuyuan). Celui qui parle ainsi pense que le Chengxin tang est un des palais des Tang du Sud, à Nankin et non à Xin'an, sans savoir que ce papier était un produit de tribut pour le souverain, et que pour cette raison il a pris le nom du Chengxin tang; mais cet endroit n'était pas un lieu de production de papier. Ainsi, le papier de Xuan sur lequel était imprimé le sceau « Papier Jasmin de Xuande », comment aurait-il été produit au Palais ?

Le Sheshi xiantan introduit ici une théorie qui voudrait que le papier de Xuan désigne, à l'origine, le papier de l'ère Xuande, donc de l'empereur des Ming Xuanzong (r. 14261436). Cette idée, qui a été motivée par des sources écrites, telles que le Feifu yulüe 飛臼語略 (Propos sommaires du canard en vol) de Shen Defu 沈德符 (1578-1642), circule encore de nos jours ${ }^{162}$. Elle n'est pas acceptée communément, mais elle sert à justifier les affaires des centres - nombreux - éloignés de l'Anhui où l'on fait du papier dit xuan : l'appellation ne serait plus géographique si l'on accepte cette définition. Évidemment, cela a été déjà réfuté par Xu Changyao, qui sépare le lieu de production et le fait que l'empereur ait apprécié ce papier au point de lui attribuer un sceau avec le nom de l'ère. Ensuite, le texte se concentre sur le Chengxin tang zhi en donnant une version très locale, je dirais familiale, des faits. Bien sûr, cette anecdote a peu de poids. Mais du moment que les sources officielles sont très limitées et vagues, elle présente l'intérêt de nous faire découvrir le point de vue des intéressés, il y a environ un siècle.

De plus, mon maître Zhou Zaibai 周再白 ${ }^{163}$, nom taboué Hong 洪, était bachelier (zhusheng) à Jixi ; parmi ses ancêtres, certains avaient servi pendant les Tang du Sud ${ }^{164}$. Ils ont transmis de père en fils que Zaigong [周載公, Zhou Zai ?] était l'auteur de beaux poèmes appréciés par son souverain, qui lui a offert du papier de Chengxin tang de différentes sortes, produit à She, et des pierres de Duan[xi 端溪?] grandes et petites. Ainsi, les papiers étaient produits à She et n'étaient pas d'une seule qualité ${ }^{165}$.

162. De nombreuses sources, anciennes ou contemporaines, parlent d'un sceau datant de la cinquième année Xuande (1430, 宣德五年造素繤纸印) ; voir aussi Shen Defu, Feifu yulüe, «Congshu jicheng 》, Shanghai, Shangwu yinshuguan, 1937, p. 8-9.

163. Bao Yilai $(2005$, p. 131$)$ attribue cet extrait du Sheshi xiantan à Huang Chongxing 鱼崇惺 (1821-1908), auteur du Junzhi bianzheng 郡志辨証, qui est un autre ouvrage cité par Xu Chengyao (vol. 1, p. 574 et suiv.), mais il me semble qu'ici la personne qui prend la parole n'est pas Huang, mais l'un des Jiang (voir note 161).

164. L'arrivée de Zhou à Jixi pendant les Tang du Sud est confirmée dans des histoires familiales, par exemple le Jixi Zhoushi zupu 續溪周氏族譜 de Zhou Wenhua 周文化 du milieu du xve siècle, où l'on parle de l'ancêtre fondateur du lignage sur place, Zhou Yao 周圭; cf. l'original de la généalogie conservé à la Bibliothèque nationale de Pékin, voir aussi le Zhongguo jiapu zongmu 中國家譜總用 (Shanghai tushuguan éd.), Shanghai Shanghai guji chubanshe, 2008, vol. 3, p. 1566, n. 199-0611, selon lequel l'ancêtre fondateur aurait d'abord servi à She dans les années 880 , avant de démenager à Jixi pendant les désordres de la fin de la dynastie des Tang.

165. Le texte s'achève par une « Note : A cette période She était une préfecture, bien que l'on ne sache pas de quel district venait le papier, et qu'on l'attribue généralement à la commanderie de Xin'an, c'est une citation du Shezhi, donc acceptable. " Autrement dit, par le passé She était le nom de toute la préfecture, et l'appellation convient donc aussi pour des districts voisins. 


\section{Le papier Xuan de Jingxian}

Pour clore cette présentation, nous avons presque l'obligation de réunir quelques informations à propos du papier Xuan de Jingxian. Car ce produit a été élu sur les listes représentatives du Patrimoine culturel immatériel de l'humanité en 2009 : il va sans doute finir par rester emblématique de toute cette production, variée et millénaire, que nous avons évoquée pour la région. Nous ne nous attarderons cependant pas trop sur sa production, ni sur les caractéristiques et les applications du produit, notamment dans la peinture, les arts graphiques et la restauration. Ces thèmes ont déjà fait couler beaucoup d'encre, et ils mériteraient certes une monographie faisant appel à de multiples compétences. Demeure aussi la difficulté d'enquêter sur ce terrain qui est passé de la fermeture aux étrangers (et plus généralement aux visiteurs extérieurs) à une " politique d'ouverture » : le plus important centre de production du papier de Xuan est aujourd'hui une destination de tourisme organisé ${ }^{166}$, dans un processus parallèle de rentabilité économique et de muséification.

Les habitants auraient pris l'habitude d'être méfiants, après que les secrets de fabrication de leur papier eurent été « volés » en 1883. L' « espion » soupçonné, Inoue Nabunasa 井上陳政 (1862-1900, nom chinois Chen Zide 陳字得), est l'auteur des notes les plus anciennes et les plus complètes à propos du papier de Xuan ${ }^{167}$, car il ne se limita pas à décrire les procédés de fabrication, mais aussi les objets et les matériaux utilisés, dont il préleva des échantillons. Les recherches sur le papier d'Inoue, menées pendant six ans à partir de $1882^{168}$, ne sont qu'un volet de ses activités sur la Chine ; il se limita à rédiger un rapport sans jamais en faire un vrai livre. Ces enquêtes furent probablement motivées par le fait qu'il avait été formé dans le bureau d'impression de la province d'Ōkura 大藏省印刷局; mais on ne peut évidemment pas exclure qu'elles puissent avoir intéressé les autoriés japonaises, qui finançaient partiellement les voyages d'Inoue.

Les contributions d'Inoue les plus importantes portent sur le papier de Xuan et du papier de bambou, très appréciés pour la peinture et la calligraphie, et ainsi différentes, par leurs propriétés, du papier produit au Japon, très souvent fait d'écorces. La spécificité du papier de Xuan réside essentiellement dans un des produits de base, communément

166. Le site internet officiel de la Compagnie à responsabilité limitée du papier Xuan de Chine (中国 宣纸股份有限公司 http://www.hongxingxuanpaper.com.cn/default.asp), consulté le 20/01/2014, est extrêmement édifiant pour une lecture au premier et au deuxième degrćs, de cet cndroit créé en 1951 à Wuxi 烏溪. La compagnie compte à présent 1200 employés et se targue dans sa présentation en ligne d'avoir déjà dépassé les 85000 visiteurs sur le lieu de production du fameux papier de la marque à l'《étoile rouge » (Hongxing 红星), après l'ouverture officielle au tourisme en 2007 ; d'autres sites de production du xuanzhi étaient déjà accessibles auparavant.

167. Mes recherches à ce propos sont pour le moment insuffisantes pour en tirer quelque conclusion que ce soit. Pour cette synthèse, je me suis basée sur l'article de Chen Gang 陳剛, «Inoue Nabunasa yu Qingguo zhizhifa " 井上凍政與清國制紙法, Shilin, 2012, 3, p. 128-132. Chen retrace l'histoire du rapport d'enquête d'Inoue : il est d'abord imprimé en polycopie (? youyin 油印), puis reproduit par copie manuscrite pour en assurer la transmission. Le texte est ensuite publié dans des périodiques, avant d'alimenter les travaux d'autres spécialistes. Les enquêtes d'Inoue à Jingxian précèdent d'une vingtaine d'années celles d'autres Japonais, et de presque un demi siècle les travaux des Chinois, tels que Zhang Yonghui 張永惠 (vers 1937). En confrontant les informations d'Inoue et de Zhang, Chen constate une simplification des procédés techniques déjà en œuvre entre la fin des Qing et la période républicaine ; par exemple, trois « cuissons » à la place de quatre auparavant. Ou encore, l'exposition au soleil qui, selon le plus ancien témoignage, devait se faire sur des terrains différents, d'abord en plan et ensuite sur le relief; mais cette précision manque dans le rapport d'enquête successif. Ibid., p. 131 .

168. Inoue voyage dans des nombreuses régions du Sud-Est de la Chine ; à Xuan, il visite des producteurs de la famille des Cao 曹; ibid., p. 129. 
appelé « le santal vert » qingtan 青檀 ${ }^{169}$, et dans la capacité des papetiers à le mélanger, avec un dosage aux proportions équilibrées, à la «paille de riz» (daocao 稻草). Sur ce point, rappelons un commentaire de Pan Jixing qui a le mérite d'aller à l'encontre d'un lieu commun encore largement repris sur ce papier de Xuan, fréquemment décrit comme un papier unique de la meilleure des qualités ${ }^{170}$.

... la "paille de riz" 171 n'a pas les qualités de l'écorce des mûriers ni du chanvre, ainsi quand on la substitue à l'écorce du mûrier à papier, en raison de la petite taille des fibres, on réduit la résistance du papier. D'ailleurs les petites taches jaunes sur les manuscrits du Siku quanshu, écrits sur papier de Xuan pendant l'ère Qianlong, se sont produites à cause de la "paille de riz"... ${ }^{172}$

Selon les sources officielles, la recette de ce papier comporterait, de nos jours, « 108 étapes » de préparation ${ }^{173}$; elle est assez semblable aux procédés utilisés pour le papier d'écorce du Jiangxi et de Huizhou. Il y a presque un siècle, Hu Yunyu 胡輼玉 (1878-1947) a décrit cette méthode de fabrication en conclusion de son «Discours sur le papier », inclus dans le recueil Puxue zhai congkan 樸學齋叢刊 (Recueil du studio des études critiques) et publié en $1923^{174}$. Son supplément, le «Discours sur le papier de Xuan » s'ouvre ainsi :

Le district de Jing faisait autrefois partie de Xuanzhou, sa production de papier est la meilleure au monde et on l'appelle papier de Xuan. Récemment apprécié partout, de l'intérieur du pays jusqu'aux mers orientales, il est considéré comme un produit de qualité pour la calligraphie et la peinture. Le papier de Xuan est exporté chaque année pour un montant estimé à plus d'un million de yuan. Ce petit chiffre ne mérite que peu de louanges, mais en considérant que c'est la production d'un district unique, alors c'est important. Dans le domaine de la forêt des arts, c'est insurpassable et suffisant pour en être fier. Malheureusement les artisans ne manient pas pinceaux et encre, et les lettrés n'ont

169. Cette plante de la famille des ulmacées est le Pteroceltis tatarinowii Maxim, unique en son genre et endémique en Chine ; Fèvre \& Métaillé, 2005, p. 367. La longueur de ses fibres, savamment associée à la petite taille des fibres de la paille, permet d'obtenir un papier résistant tout en gardant sa souplesse.

170. C'est le cas dans le récent article de Cen Deling, « Le papier xuan dans la restauration des peintures et calligraphies chinoises », Arts Asiatiques 68,2013, p. 87-90, qui présente de jolies photos de l'usine de Hongxing 紅星; son discours n'est pas dépourvu de généralités « esthétisantes ».

171. J'ai introduit des guillemets pour la paille utilisée dans la composition du xuanzhi, car ce qu'on appelle paille de riz n'est pas toujours de riz, mais aussi d'herbe ; elle a surtout pour fonction d'assouplir la feuille, mais si sa composante est trop élevée, le papier perd alors de sa résistance et ses qualités au contact de l'encre sont réduites.

172. Pan Jixing, 1998, p. 250. Tous les auteurs ne partagent pas cette idée. Polastron explique que certaines taches présentes sur les beaux papiers seraient un effet des eaux de montagne, avec un excès en apport de particules ferriques (2010, p. 145).

173. Du moins selon le document de la convention pour la sauvegarde du patrimoine culturel immatériel pour l'inscription sur la liste représentative en 2009 (référence $n^{\circ} 00201$ ), sur le site de l'Unesco. Il est cependant impossible d'identifier les 108 étapes à partir des documents dont je dispose.

174. Le congshu est publié en 1923 (douzième année de la période républicaine) et le traité sur le papier est un des onze textes qui le composent. Le passage traduit ci-dessous est le complément final, après des sections plus générales sur les origines et l'histoire du produit, les matériaux utilisés et les différentes qualités, la présentation des données de façon chronologique et géographique, les techniques de productions et les noms de producteurs depuis Cai Lun 蔡倫 (50-121). 
pas encore fait de mémoires [à ce sujet], et bien que la valeur du papier soit grandement appréciée, personne n'en a narré les tenants et aboutissants. Sans dessein [particulier], j'ai humblement pris les notes ci-dessous, sans pouvoir traiter [le sujet] en détail. Selon le Zhenzhu chuan 珍珠船 de Hu Shi 胡侍 (1492-1553) ${ }^{175}$, pendant l'ère Yonghui (650656), un moine de Xuanzhou désirait écrire le Huayan jing 華嚴經 (Avatamsaka sūtra ou Sütra de la guirlande de fleurs) et il remplaça le bois d'aloès (Aquilaria agallocha $R o x b$ ) par une plantation d'arbres de mûrier à papier pour en faire du papier, et ainsi a commencé la production du xuanzhi. À Xuancheng, Ningguo, Jingxian et Taiping, partout on savait faire du papier, d'où son nom de xuanzhi; mais c'est à Jingxian que la production était la meilleure ${ }^{176}$. Aujourd'hui il n'est produit que dans le district de Jing et c'est pour cette raison qu'on l'appelle aussi Jingxian zhi. (Le Zhuanghuangzhi 裝㣴志 de Zhou Jiazhou 周嘉虫 (1582-1658) dit que [pour le montage] il faut choisir du liansi 連四 de Jingxian, ou du dangong 單供, ou encore des matériaux de bambou. Selon le Changwuzhi 長物志 de Wen Zhenheng 文震宁 (1585-1645), parmi les papiers de Wu 吳 il y avait le sajinjian 灑金箋 et aussi le tanjian 譚嘎 de Songjiang, mais tous ne résistaient pas longtemps et le liansi de Jingxian était le plus précieux ${ }^{177}$ ). À Jingxian, la zone de production du papier comprend Fengkeng 楓坑, et elle s'étend jusqu'à Xiaoling 小領 et Nikeng 泥坑 près de Caoxi 漕溪. Parmi les producteurs, les Cao 曹 et les Zhai 㛭 sont les plus nombreux. Dans des lieux autres que Jing(xian) on produit du papier, mais seuls les gens de Jingxian savent le faire! Ses qualités sont multiples, selon les monographies locales... ${ }^{178}$

...[Parmi ces qualités de papier] le huaxin 畫心 suit exactement la méthode transmise du Chengxin tang; adapté aux calligraphies et aux peintures, il est précieux dans le jardin des arts. Ses dimensions atteignent deux, huit, six et quatre chi; son épaisseur peut être simple, double ou triple. Les matériaux utilisés varient de la pure écorce, à la moitié, ou encore ou sept (dixièmes) d'écorce et trois (dixièmes) de riz. Pour faire le papier, l'essentiel est dans la matière ; comme matière, on utilise l'écorce du mûrier à papier ou du santal (vert) qui doivent avoir poussé sur des reliefs rocheux hérissés et pentus pour devenir des matériaux de qualité. Au milieu de l'hiver, pendant le douzième mois, les hommes qui habitent ces lieux brisent les branches de ces arbres, les découpent et les font cuire à la vapeur. On détache ainsi l'écorce, on la fait blanchir avec l'eau des ruisseaux et de la chaux pendant plus de dix jours ou vingt jours, selon les cas. La substance de l'écorce se dissout et ce qu'on en retire est pilé. Les pilons sont activés à l'eau, ils fonctionnent ainsi et les hommes restent à côté pour contrôler. Dès que (les fibres) se défont, de nouveau on blanchit la pâte et on la pile, en tout trois, quatre fois ; on

175. En fait Hu Shi est l'auteur de Zhenzhu chuan 真珠船 et Chen Jiru 陳繼儒 (1558-1639) du Zhenzhu chuan 珍珠船 où se trouve la citation, un deuxième problème étant que dans les versions que j'ai consultées, le moine est dit être de Dingzhou 定州! Cf. Chen Jiru, Zhenzhu chuan, dans Baoyan tang miji 䩀顏堂秘策, Shanghai, Wenming shuju, 1922, ch. 1, p. 5v; "Congshu jicheng chubian ", Shanghai, Shangwu yinshuguan, $1936:$ Zhenzhu chuan, p. 10.

176. Le texte renvoie ici au Wanling junzhi 宛陵郡志, mais je n'ai pas identifié cet ouvrage.

177. Zhou Jiazhou, Zhuanghuangzhi, « Congshu jicheng chubian », Shanghai, Shangwu yinshuguan, 1939, p. 9. Pour Wen Zhenheng, voir plus bas, note 185. Ce passage entre parenthèse est présenté comme commentaire - écrit en petits caractères, dans le Puxue zhai congkan, op. cit., p. 14 v.

178. Au milieu du texte il y une énumération de noms de papiers, mais comme ceux-ci sont tirés de sources de l'administration locale, nous les citons par la suite à propos des monographies locales, voir notes 190-191. 
élimine les impuretés et on conserve cette substance fluide. On prend du jus d'actinidia chinensis ${ }^{179}$ que l'on a fait infuser, on l'introduit dans la cuve et on mélange de façon uniforme. Utilisant un treillis de bambou fin porté par deux hommes, il est retiré (de la pâte) une, deux ou trois fois pour obtenir autant d'épaisseurs. Une grosse quantité (de feuilles) est entassée et pressée. Quand toute l'eau est évacuée, on pose (les feuilles) sur un objet façonné en argile avec du feu (à l'intérieur), les feuilles sont pressées et, une fois retirées, on les expose dans un lieu au soleil et au vent, et ainsi se fait le papier. »

Quelques éléments de ce bref exposé sont à retenir, notamment les réflexions qui montrent que la personne qui écrit vit au $\mathrm{xx}^{\mathrm{e}}$ siècle. Hu est conscient que ce "trésor » de la calligraphie et de la peinture est un produit connu hors de Chine, en Asie orientale et qui a une valeur économique non négligeable pour le district où il est produit. Il attire aussi l'attention sur le fait que les artisans ne savent pas assez bien écrire pour décrire leurs pratiques de production, et que les lettrés n'ont pas pris la peine d'en parler non plus. Il explique qu'il existe différentes qualités de produit, mais surtout différentes catégories d'un même produit selon la taille et l'épaisseur de la feuille, ou la composition de la matière utilisée. Il ne fait qu'une seule mention explicite de la paille de riz; dans son discours, cette paille, qui est de nos jours devenue emblématique du xuanzhi lui-même ${ }^{180}$, est un produit parmi d'autres, tandis que l'écorce reste indispensable. $\mathrm{Hu}$ indique aussi l'utilisation, comme additif, du jus de kiwi déjà présent à Huizhou longtemps auparavant ; en revanche, la chaux a désormais pris la place des cendres utilisées quelques siècles plus tôt.

Un dernier sujet à retenir, à partir de travaux ayant une approche historique ${ }^{181}$, est l'introduction de la production de ce papier à Jingxian, que l'on fait remonter à la fin des Song. Un certain Cao Dasan 曹大三 (1255-1342) quitta le district de Nangling 南陵, à l'ouest de la ville de Xuan, en se déplaçant vers le sud, dans les montagnes, plus exactement à Xiaoling 小領 ${ }^{182}$ : les Cao s'installent et reprennent une activité qu'ils avaient probablement auparavant. Cette histoire, qui mérite d'être vérifiée, serait racontée dans les généalogies de la famille, notamment le Jingchuan Xiaoling Caoshi zongpu 涇川 小領曹氏宗譜 imprimé au Jishan tang 繼善堂 en $1872^{183}$. La production de papier se

179. Dans ce texte, les caractères sont yangzhiteng 陽枝藤 mais il n'y a pas de raison de penser qu'il s'agit d'autre chose que de la plante décrite plus haut, notes 75 et 114 .

180. Il suffit de s'intéresser au xuanzhi pour découvrir les images de cette paille exposée au soleil pour sécher et blanchir. Ceci donne un aspect particulier au paysage, parsemé de champs avec des différents degrés de blancheur, en fonction du temps d'exposition du produit.

181. Pour cette conclusion, je me suis basée sur le livre de Pan Jixing (1998, p. 249 et suiv.), tout en étant consciente de ses limites.

182. Plusieurs endroits de ce nom dans le district de Jingxian sont repérables sur des cartes en ligne. Ils sont plus ou moins éloignés de Nanling, Jing et Wuxi ; au vu des informations disponibles, il est difficile de trancher.

183. C'est Pan Jixing qui donne ces informations en disant que la généalogie contient une préface de 1778 portant sur ce sujet. Selon le Zhongguo jiapu zongmu (2008) deux généalogies portant le titre Jingchuan Xiaoling Caoshi zongpu existent, imprimées par caractères mobiles en bois : l'une fut effectivement réalisée à l'endroit indiqué en 1873, l'autre ailleurs en 1914. Trois autres généalogies des Cao de Jingxian sont répertoriées : deux Qiaogwo Caoshi zongpu 譙國曹柇宗譜 de 1778 et 1812, dont la première pourrait contenir des informations supplémentaires, vu sa date qui coïncide avec la préface citée par Pan Jixing ; une dernière généalogie, en date de 1894, a pour titre Pingyang Caoshi zongpu 平. 陽曹氏宗譜; cf. Zhongguo jiapu zongmu, 2008, vol. 5, p. 2823, entrées de 328-0144 à 328-0148. Je n'ai 
serait perpétuée, menée essentiellement par les familles Cao et Zhai 翟 ${ }^{184}$. Le papier de Jingxian devint célèbre et les auteurs des manuels « de bon goût » de la période Ming en firent l'éloge : « le liansi de Jingxian est extrêmement précieux », écrit Wen Zhenheng 文震亨 (1585-1645) dans son Changwu zhi 長物志 ${ }^{185}$. Son contemporain Shen Defu raconte que les peintres de Suzhou du siècle précédent l'appréciaient particulièrement et l'employaient après l'avoir fait vieillir ${ }^{186}$.

Il est aussi intéressant de signaler une des rares citations dans l'encyclopédie impériale Gujin tushu jicheng 古今圖書集成 des années 1720, qui affirme que (à Suzhou) l'on faisait des tentures en papier (zhizhang 紙帳) peintes, avec des motifs de branches de prunier : “ le papier utilisé est du papier mianzhi 綿紙 de Jingxian ${ }^{187}$ ». Dans ce cas, le nom du district est associé à ce papier « cotonneux », une expression qui désigne son aspect - soyeux et blanc -, et non sa composition. Le mianzhi n'est pas un terme fréquent dans les sources concernant notre région, mais de nos jours son usage s'est banalisé pour désigner les papiers de très bonne qualité.

Sous les Qing, pendant la période républicaine ou encore de nos jours, les auteurs en viennent à «tisser des liens » entre le papier fait à Jingxian et celui de Xuan(zhou), voire avec le papier du Chengxin tang, en échangeant les termes ou en concluant que Jingxian fut parfois sous l'administration de Xuan, d'où l'appellation commune ${ }^{188}$. En réalité, les monographies locales du district de Jing et de la préfecture de Xuan sont assez avares d'informations sur ce sujet. Le Jingxian zhi des Qing ${ }^{189}$ ne fait qu'une énumération de produits : dix papiers faits d'écorce, dont « un qui est aussi appelé Chengxin tang » ${ }^{190}$, deux papiers de bambou et trois de paille ${ }^{191}$. On constate aussi que des objets comme le parasol ou le parapluie sont fabriqués en papier d'écorce ; quant aux yizhi (vêtements)

pu que consulter rapidement un exemplaire du Xiaoling Caoshi zongpu 小領曹氏宗譜 du Yuqing tang 餘慶堂, compilé par Cao Hongkui 曹鸿逵, qui se trouve à la Bibliothèque de la Columbia University à New York : East Asian Special Collection 2252.95560 .32 f. Selon cette généalogie qui comprend une préface de 1914, Cao Dasan (1264-1342) arriva à Xiaoling à la fin de la dynastie des Song, pour fuir les désordres mais aussi attiré par la bonne situation des lieux (宋末時游至涇川, 見小領L水秀麗風土).

184. Quatre généalogies des Zhai (réalisées entre 1767 et 1893), toutes faisant mention de Shuidong 水東 dans le district de Jingxian, sont répertoriées dans Zhongguo jiapu zongmu, 2008, vol. 7, p. 4170, entrées de 497-0011 à 497-0014.

185. Cf. Wen Zhenheng, Changwu zhi jiaozhu 長物志校汸., [s.1.] : Jiangsu kexue jishu chubanshe, 1984, juan 7, p. 307.

186. Cf. Shen Defu, p. 9.

187. Cf. Chen Menglei 陳夢靁 (éd.), Gujin tushu jicheng, version en ligne sur le site de l'université de Harvard, consultée le 20/01/2014: «Suzhou fu wuchang kao »蘇州府物產考.

188. Cen Delin, 2013, p. 87.

189. Cette monographie a été imprimée deux fois sous les Qing et une fois pendant la période républicaine : cf. Li Degan 李德淦, Hong Liangji 洪亮吉, Jingxian zhi, «Zhongguo fangzhi congshu : Huazhong difang ", Taipei, Chengwen chubanshe, 1975, p. 511.

190. Cette liste énumère : jinbang 金枋, luwang 路王, bailu 白鹿, huaxin 甾心 qui est aussi appelé chengxin tang, luowen 羅䋌, juanlian 卷䬼, liansi 連四, gong[dan] 公[單], xueshu學書 et sanzhi 金紙 (parasol en papier).

191. Ce sont le ganzhang 古張 et le huozhi 火紙, ainsi que le xiabao 下包, gaolian 高廉 et yizhi 衣紙 (papier pour vêtements ?); sur le huozhi, voir plus haut, p. 299-300. 
en papier de paille, il est légitime de se demander s'il s'agissait de quelque chose de comparable aux descriptions anciennes, ou plutôt à des objets rituels ${ }^{192}$.

Exactement les mêmes papiers de paille et de bambou sont cités dans le Xuancheng xianzhi (宣城縣志, 1888) ${ }^{193}$. Cette monographie du district de Xuancheng est dépourvue en revanche de la liste de noms des papiers d'écorce de l'arbre de santal (tanshupi 檀樹皮), qui se trouve dans le Jingxian zhi. Mais, ici, il est précisé que ce papier est appelé xuanzhi, et que tous savent le produire à Xuan(zhou), Ning(guo), Jing(xian) et Tai(ping ?). Enfin, cette monographie transcrit un poème d'un auteur local des Qing, Zhao Tinghui 趙廷揮, en quatre vers de sept caractères : «À quoi s'occupent les foyers dans les montagnes? Ils sont occupés en nombre à faire tourner les pierres (des moulins). Des liasses (de papier forment) des murs nouveaux, et près des ruisseaux les piles ne s'arrêtent jamais. Un coup de pilon résonne et fait trembler le soleil couchant. "

\section{Conclusion}

Même si la partie conclusive de cet article propose quelques informations à propos d'une qualité de papier qui est aujourd'hui valorisée au nom du patrimoine de l'humanité et pris comme symbole de la culture et des arts chinois, cette recherche visait à retracer, à partir des sources historiques et des documents de l'administration provincialc, l'histoire d'un produit local. En poussant l'exercice à son extrême, nous espérons avoir décrit un cas susceptible d'apporter un éclairage à une plus vaste échelle, d'autant plus que l'importance « économique » de ce produit a toujours dépassé son territoire de production. Car le territoire correspondant à la préfecture de Hui est évidemment dans un rapport d'échange, et parfois de concurrence, avec d'autres localités productrices de papier, toutes situées dans cette vaste région au croisement des actuelles provinces de l'Anhui, du Zhejiang et du Jiangxi, où des conditions climatiques et géographiques étaient favorables à la réunion des matières nécessaires à la fabrication. La production du papier reste plus liée que d'autres manufactures au monde rural : grande consommatrice de végétaux et d'eau, relativement simple par rapport à d'autres techniques, complémentaire à d'autres activités agricoles, cette manufacture avait des difficultés objectives à s'implanter dans les villes ou les capitales. Il n'est d'ailleurs pas impossible que la presque disparition de la production du papier d'écorce à Huizhou pendant les périodes plus récentes, soit tout simplement liée à un manque de matière première, à cause d'une surexploitation ou de sa substitution par d'autres cultures.

Dans les différents exemples proposés, nous avons retrouvé quelques constantes que les techniques du papier partagent avec d'autres productions également pratiquées par des artisans anonymes, comme l'implication de familles, génération après génération, dans la transmission de connaissances pratiques; ou encore, la mobilité de ces derniers, et des marchands, comme probable facteur de transmission des techniques elles-mêmes ${ }^{194}$. Ces artisans-papetiers «ne maniaient pas pinceaux et encre » : ils n'ont donc pas laissé d'écrit concernant l'histoire ou la transmission des techniques qu'ils maîtrisaient. Enfin,

192. Voir plus haut, p. 282-283 et 298.

193. Li Yingtai 李應泰, Zhang Shou 章綬, Xuancheng xianzhi, «Zhongguo fangzhi congshu : Huazhong difang. Anhui sheng », Taipei, Chengwen chubanshe, 1985, p. 272.

194. La question de l'anonymat des artisans et de l'importance de la mobilité des populations dans la diffusion des techniques est avancée à plusieurs reprises dans différentes contributions du volume édité par M. Schäfer, op. cit., 2012. 
tandis que la littérature technique chinoise, plus ou moins spécialisée, a tendance à associer des compétences à des personnalités précises ${ }^{195}$, historiques et mythiques, souvent exemplaires, leurs noms manquent à notre récit : ce fait n'a certes pas contribué au développement d'une littérature à ce propos.

Ainsi nous comprenons mieux l'intérêt de ces quelques textes, inclus dans les monographies locales, qui nous transmettent quelques techniques anciennes utilisées par des gens du commun pour produire du papier. Le procédé est toujours décrit globalement, sans distinction par rapport à d'autres activités qui pourraient être parallèles et spécifiques (s'occuper des mûriers à papier, traiter leur écorce, préparer les outils nécessaires pendant la production...). On n'y fait pas non plus mention de compétences spécialisées de certains groupes de personnes : est-ce que les hommes et les femmes avaient des rôles spécialisés, comme on le constate des nos jours dans les manufactures de type traditionnel ${ }^{196}$ ? Quand le travail nécessitait la réunion d'un nombre important de personnes - par exemple pour le papier de très grandes dimensions comment les réunissait-on et à quelle occasion ${ }^{197}$ ?

Néanmoins, dans le contexte chinois, malgré leur récit parfois incomplet, ces textes restent précieux, en raison de leur relative rareté. Ils ont bien évidemment été composés par des fonctionnaires, qui détiennent d'ailleurs le monopole de la littérature technique ancienne. Mais ils décrivent ici des productions ou des objets faits en papier qui nous éloignent du monde des lettrés, ou mieux finissent par donner une image plus nuancée et élargie de celui-ci - nous préservant en partie de la rhétorique répétitive à propos des « trésors du lettré »- et nous conduisent tant à la campagne que sur des marchés, dans une cour impériale comme dans le yamen d'un petit bourg.

Les exemples analysés explicitent aussi les enjeux économiques, au moins pour la première partie du deuxième millénaire, indiquant la place que le papier trouvait parmi les produits locaux de valeur et les tributs prélevés annuellement. Cette intervention de l'État via la fiscalité montre aussi que l'administration se souciait de récupérer au mieux une production qui paraît comme étant faite en dehors de son organisation, au moins dans l'Anhui sous les Song. Cette situation aurait changé sous les Yuan quand, au moins au début de leur règne, le travail des artisans est organisé directement « dans » l'administration, par la gestion de foyers, même si cela ne semble pas avoir fonctionné durablement à Huizhou : les conditions étaient-elles trop dures ou les compensations insuffisantes? Cette dernière hypothèse qui présuppose une commercialisation privée

195. Le cas de Cai Lun (vers 50-121), inventeur du papier, est un exemple de cette pratique ; le culte de ce personnage a été perpétué à l'époque contemporaine, avec des conséquences par moments lourdes pour le développement de la discipline, comme le raconte Pan Jixing dans « Review on the debate of paper history during recent 30 years in China ", Journal of the International Association of Paper Historians, vol. 15-2, 2011 , p. 6-12 ; ce sujet a été évoqué par Jean-Pierre Drège, dans son intervention « Les controverses concernant les origines du papier en Chine ", D'Est en Ouest : relations bilatérales autour. du papier entre l'Extrême-Orient et l'Occident ( 10 octobre 2014). Il existe par ailleurs une version de l'histoire qui présente un disciple de Cai Lun, Kong Dan 孔ff de la période des Han, comme étant celui qui découvrit le papier de Xuan ; je n'ai cependant retrouvé cette histoire que dans des sources modernes.

196. C'est notamment le cas pour certains papiers encore produits au Japon : le nettoyage de l'écorce pour les qualités les plus précieuses est traditionnellement une tâche féminine. Voir le papier de Echizen décrit par É. Orsenna, op. cit., 2012, p. 94-95.

197. Pour cette production, Wei Huaxian (2006, p. 141) arrive à imaginer une forme de travail coopératif de plusieurs ateliers ; voir plus haut note 70 . Sans avoir aucun élément pour le contredire à propos des Song, nous ne pouvons pas ignorer le développement du système clanique à Huizhou : réunir plusieurs membres d'une seule famille n'était pas impossible. On peut également se demander à quelle échéance se faisait ce papier hors-échelle, pour une production régulière ou à des occasions particulières pendant des réunions de la communauté ? 
du papier produit, nous laisse imaginer l'intervention d'une autre catégorie d'acteurs économiques, également peu représentés dans la littérature mais certes très importants : les marchands et les commerçants.

L'ensemble des informations pour les dynasties des Song et des Yuan permet de comprendre comment le papier, décliné en différents types, était à la fois banalisé et indispensable pour l'administration impériale et son énorme structure bureaucratique, mais aussi pour la monnaie de l'Empire. Car, comme avait pu le remarquer un témoin plus que célèbre déjà à la fin du XIII ${ }^{\mathrm{e}}$ siècle, son souverain " [...] quant ces chartretes sont faites [...], si en fait faire tous ses paiements. Et les fait despendre à chacun par toutes ses provinces, et par tous ses règnes et par toutes ses terres, et partout où il a pouvoir... $1{ }^{198}$.

Quant aux deux dernières dynasties, si les sources de première main des archives locales sont (trop) rares, les quelques informations glanées dans différents documents ont été réunies afin de compléter un discours qui comporte souvent une approche uniquement qualitative, insistant sur les propriétés esthétiques et les emplois artistiques de ces précieux papiers. Ces caractéristiques sont certes très importantes, d'autant plus que les plus célèbres lettrés des Song, puis des Ming, se sont appliqués aux compositions littéraires les plus variées - des notes, des poèmes, des manuels - pour les célébrer. Mais nous avons également cherché à rester proche des ateliers et des cuves éparpillés dans le territoire montagneux situé à l'extrémité méridionale de la province de l'Anhui, ainsi que les lieux de vie et de travail évoqués en poésie dans la dernière des monographies citées.

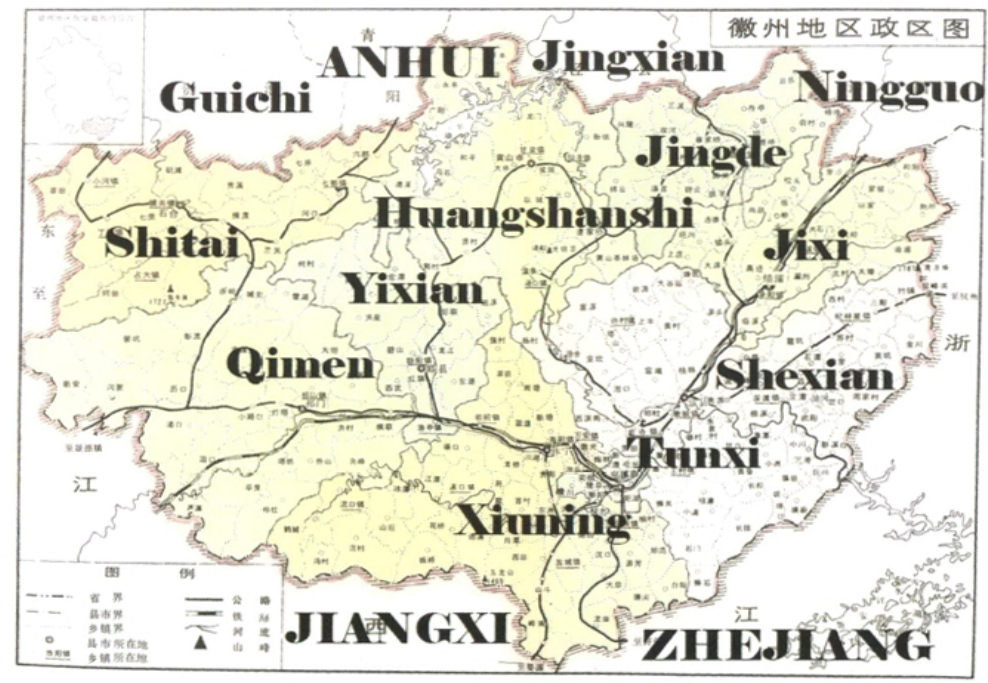

Carte 1 : Carte contemporaine de Huizhou, avec de nouveaux territoires au nord mais sans Wuyuan, inclus quant à lui dans la province du Jiangxi (carte extraite de Huizhou diqu jianzhi, s.l., Huangshan shushe, 1989).

198. Le livre de Marco Polo citoven de Venise... par M. G. Pauthier, Paris, typographie de Firmin Didot frères imprimeurs de l'Institut de France, 1865, ch. XCV, p. 323 : le mot "chatretes" indique ici le papier-monnaie. À propos du papier-monnaie des Yuan et des témoignages de M. Polo, voir le récent livre de H. U. Vogel, 2013. 


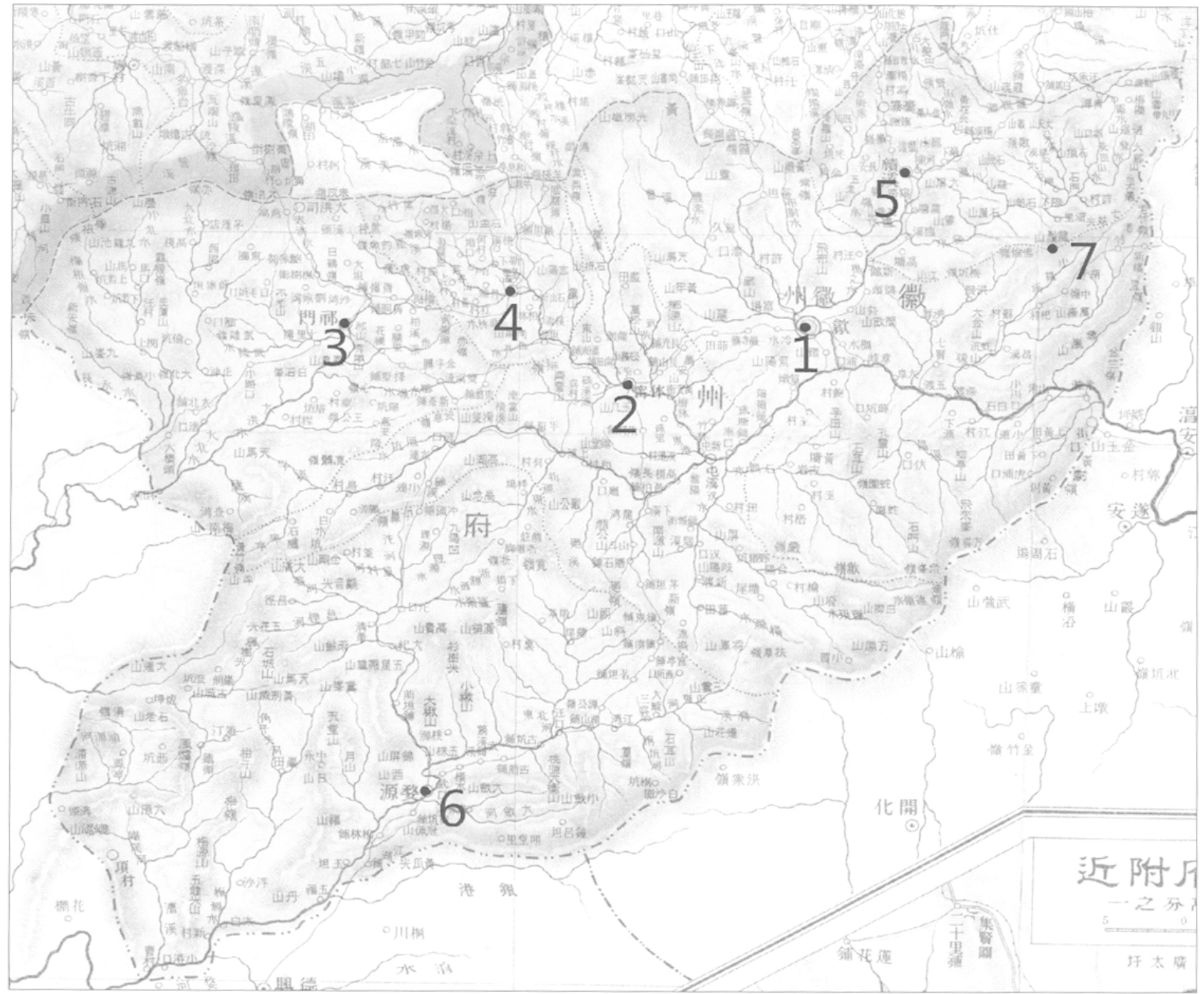

Carte 2 : Préfecture de Huizhou au début du $x^{\mathrm{c}}$ siècle à partir d'une carte de la période républicaine (reproduction extraite du volume 13 de Sinologie française, 2010).

1. Shexian - 2. Xiuning - 3. Qimen - 4. Yixian - 5. Jixi - 6. Wuyuan - 7. Longxu (entre Shexian et Jixi).

Par cette carte, on comprend que Wuyuan (6) ait pu être détaché de la circonscription administrative pour intégrer la province du Jiangxi ; d'ailleurs, ce lieu n'apparaît pas dans notre récit. 


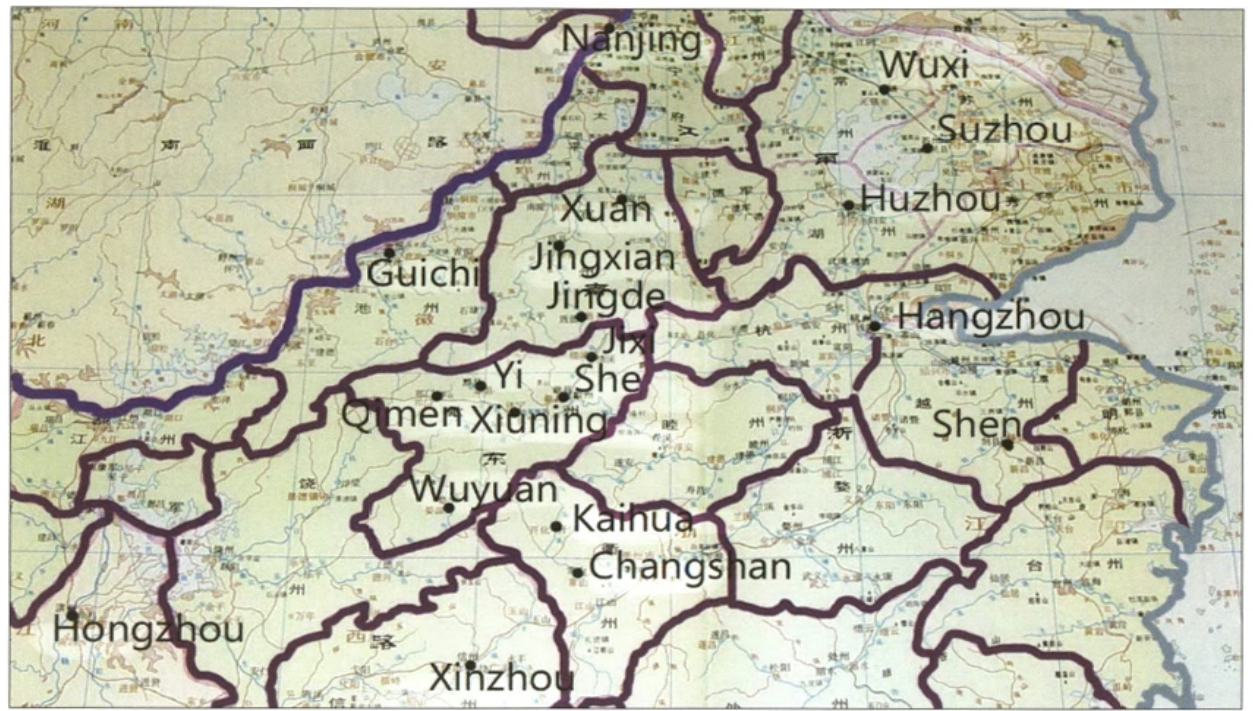

Carte 3 : Le sud de l'Anhui et les régions orientales limitrophes (carte fondée sur une carte historique de la période des Song, extraite de Zhongguo lishi ditu ji, Beijing, Zhongguo ditu chubanshe, $1982 ; 3^{\mathrm{e}}$ édition, 1996).

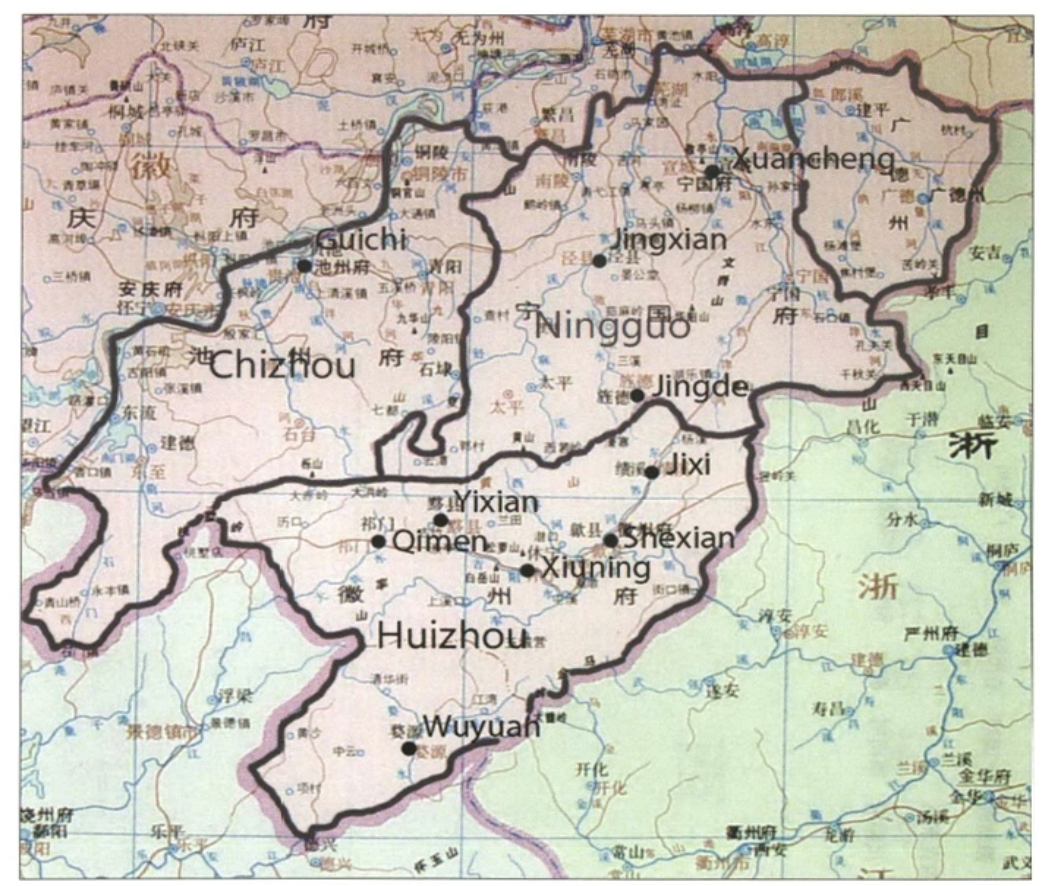

Carte 4 : Carte de Huizhou, Chizhou et Xuanzhou (Ningguo) (carte historique de la période des Qing, extraite de Zhongguo lishi ditu ji, Beijing. Zhongguo ditu chubanshe. $1982 ; 3^{e}$ édition, 1996). 


\section{BIBLIOGRAPHIE}

\section{Abréviations}

HF-HZ, Huizhou fuzhi (ère Hongzhi), voir PENG Ze \& WANG Shunmin HF-JJ, Huizhou fuzhi (ère Jiajing), voir HE Dongxu \& W ANG Shangning SHY, Song huiyao jikao, voir XU Song

SKQS, «Wenyuan ge Siku quanshu dianziban », voir YoNG Rong

SS-SKQS, Songshi, voir TUOTUo

XAZ-EC ; XAZ-F ; XAZ-SKQS, voir Luo Yuan

YJZ, Yuanfeng jiuyu zhi, voir WANG Cun

\section{Sources}

CAI Xiang 蔡襄 (1012-1067)

Wenfang sishuo 文房四説, édition en ligne : http://site.douban.com/111253/widget/ notes/1317348/note/128255594/.

CAO Hongkui 曹鴻迕 (s.d.) et al.

1914 Xiaoling Caoshi zongpu 小領曹氏宗譜, édition du Yuqing tang 餘慶堂, 24 volumes.

CAO Zhao 曹昭 (1330-1400 environ) et al.

1939 Xinzeng gegu yaolun 新增格古要論, 《Congshu jicheng chubian 》䠫書集成 初編, Shanghai, Shangwu yinshuguan.

CHFN Jiru 陳繼儒 (1558-1639)

1922 Baoyan tang miji 珼顏堂秘笈, Shanghai, Wenming shuju.

1936 Zhenzhu chuan 珍珠船, "Congshu jicheng chubian », Shanghai, Shangwu yinshuguan.

CHEN Menglei 陳夢雷 (1650-1741) et al.

Gujin tushu jicheng 古今圖書集成, «Zhonghua wenming baike quanshu » 中華文 明百科全畫, édition en ligne : http://greatman.eastview.com.ezp-prodl.hul. harvard.edu/Chinesebookweb/home/index.asp.

Cheng Chunyu 程春宇 (fin des Ming)

2007 Shishang leiyao 士商類要 (copie photostatique), Paris, Bibliothèque de l'Institut des hautes études chinoises.

FAN Chengda 范成大 (1126-1193)

1966 Canluan lu驂鸞錄, «Conghu jicheng jianbian», Taipei, Shangwu yinshuguan.

FE.l Zhu 費著 (1303-1363 environ)

1936 Shujian pu 蜀茂譜, 《Meishu congshu » 美術叢書, Shanghai ; reproduit en 1986, Yangzhou, Jiangsu guji chubanshe.

GAO Lian 高濂 (actif : 1580-1600)

1979 [?] Zunsheng bajian 遵生八茂, Taipei, Shangwu yinshuguan.

Guangyu kao, voir WANG Fengyu. 
HE Dongxu 何東序 (Ming) \& WANG Shangning 汪尚寧 (Ming)

1998 Huizhou fuzhi 徽州府志 (Monographie de Huizhou, éd. de l'ère Jiajing), dans Beijing tushuguan guji chuban bianji zu 北京圖書館古籍出版編輯組 (éd.), “ Beijing tushuguan guji zhenben congkan 》北京圖書館古籍珍本叢刊, vol. 29 (Shibu 史部 dililei 地理类), Beijing, Shumu wenxian chubanshe [abrégé en HF-JJ].

HoNG Mai 洪邁 (1123-1202)

Yijianzhi 夷堅志, SKQS ; autre version en ligne : http://blog.sina.com.cn/s/ blog_6a5826b60102e2w8.html.

HoNG Mai 洪邁 (1123-1202) \& Kong Fanli 孔凡禮 (éd.)

2002 Rongzhai suibi 容齋隨筆, Beijing, Zhonghua shuju.

Hu Shi 胡侍 (1492-1553)

1936 Zhenzhu chuan 真珠船, "Congshu jicheng chubian ", Shanghai, Shangwu yinshuguan.

Hu Yunyu 胡輼玉 (1878-1947)

1923 Puxue zhai congkan 樸學齋叢刊, Anwu, éd. de la famille Hu.

Jiutang shu, voir LıU Xu.

Lı Degan 李德涂 (XVII ${ }^{\mathrm{e}}$-XVIII ${ }^{\mathrm{e}}$ siècles) \& HoNG Liangji 洪亮吉 (1746-1809)

1975 Jingxian zhi 涇縣志, «Zhongguo fangzhi congshu: Huazhong difang 》中國 方志叢書 華中地方, Taipei, Chengwen chubanshe.

Li Shizhen 李时珍 (1518-1593)

2006 Bencao gangmu zhenzangban 本草綱目珍藏版, Beijing, Beijing yanshan chubanshe.

Li Tao 李壽 (1115-1184)

1979 Xu Zizhi tongjian changbian 續資治通鑑長編, Beijing, Zhonghua shuju ; édition en ligne : http://www.guoxue123.com/shibu/0101/01jzztjcp/ 037. htm [abrégé XZCB].

LI Yingtai 李應泰 (XIX ${ }^{\mathrm{e}}$ siècle) \& ZHANG Shou 章綬 (1827-1896)

1985 Xuancheng xianzhi 宣城縣志, «Zhongguo fangzhi congshu : Huazhong difang », Taipei, Chengwen chubanshe.

LıU Quanzhi 劉權之 (1698-1780) \& ZHANG Shifan 張士範 (1731-1795)

1985 Chizhou fuzhi 池州府志 (édition de l'ère Qianglong [1778]), « Zhongguo fangzhi congshu : Huazhong difang », Taibei, Chengwen chubanshe.

Liv Xu 劉昫 (887-946) et al.

1975 Jiutang shu 舊唐書, Beijing, Zhonghua shuju.

Liu Yueyun 劉獄雲 (1849-1919)

1995 Gewu zhongfan 格物中法, «Zhongguo kexue jishu dianji tonghui »中國科 學技術典籍通彙 zonghe juan 綜合卷, Zhengzhou, Henan jiaoyu chubanshe.

Luo Yuan 羅願 (1136-1184)

Xin'an zhi 新安志, SKQS [abrégé XAZ-SKQS].

1988 Chunxin Xin'an zhi 淳熙新安志, «Zhongguo fangzhi congshu : Huazhong difang ", Taipei, Chengwen chubanshe, 1970 [abrégé en XAZ-F].

2008 《Xin'anzhi》zhengli yuyanjiu 《新安志》整理與研究, XIAO Jianxin 蕭建新 (éd.), Hefei, Huangshan shushe [abrégé en XAZ-EC]. 
OUYANG Xiu 歐陽修 (1007-1072) \& SONG Qi 宋祁 (998-1061)

1975 Xin Tangshu 新唐書, Beijing, Zhonghua shuju.

PENG Ze 彭澤 (Ming) \& WANG Shunmin 汪舜民 (Ming)

1964 Huizhou fuzhi 徽州府志 (Monographie de Huizhou, éd. de l'ère Hongzhi), dans «Tianyi ge cang Mingdai fangzhi xuankan »天一閣藏明代方志選刊, Shanghai, Shanghai guji chubanshe [abrégé en HF-HZ].

SHEN Defu 沈德符 (1578-1642)

1937 Feifu yulüe 飛睤語略, dans le "Congshu jicheng ", Shanghai, Shangwu yinshuguan.

SoNG Yingxing 宋應星 (1587-1666)

1959 Tiangong kaiwu 天工開物 (1637), dans « Zhongguo gudai keji tulu congbian chuji »中國古代科技圖錄叢編初集, Beijing, Zhonghua shuju ; 1988, 《Zhongguo gudai banhua congkan »中國古代版畫叢刊, Shanghai, Shanghai guji chubanshe.

Su Shi 蘇軾 (1037-1101)

Dongbo zhilin 東坡志林, SKQS.

Su Yijian 蘇易間 (958-996)

1936 Wenfang sipu文房四譜, «Congshu jicheng», Shanghai, Shangwu yinshuguan.

Tu Long 屠隆 (1543-1605)

1936 Zhimo biyan jian 紙墨筆硯笺, “ Meishu congshu ", Shanghai ; reproduit en 1986, Yangzhou, Jiangsu guji chubanshe, vol. 2.

TUOTUo 脫脫 (1314-1355)

1997 Songshi 宋史, Beijing, Zhonghua shuju; SKQS [abrégé SS-SKQS].

WANG Cun 王存 (1023-1101)

1984 Yuanfeng jiuyu zhi 元豐九域志, Beijing, Zhonghua shuju; SKQS [abrégé YJZ].

WANG Fengyu 汪缝預 \& WANG Zuochou 汪作舟 (fin des Ming) (éd.)

1594-1595 Guangyu kao 廣舆考, Wuyuan (?).

WANG Qi 王圻 \& WANG Siyi 王思義 (fin des Ming)

1988 Sancai tuhui 三才圖繪, Shanghai, Shanghai guji chubanshe.

WANG Zhen 王禎 (1271-1333)

Nongshu 農書, SKQS.

WEN Zhenheng 文震亭 (1585-1645)

1984 Changwu zhi jiaozhu 長物志校注, CHEN Zhi 陳植 \& YANG Chaobo 楊超伯 (éd.), s.l., Jiangsu kexue jishu chubanshe.

XIE Jin 解經 (1369-1415) et al.

1997 Yongle dadian 永樂大典, “Siku quanshu cunmu congshu bubian 》, Jinan, Qilu shushe, 58-72.

2003 Yongle dadian shiqijuan: Haiwaixinfaxian 永樂大典十七卷：海外新發現, Shanghai, Shanghai cishu chubanshe.

XIE Min 謝旻 $\left(\mathrm{XVII}^{\mathrm{c}}\right.$-XVIII ${ }^{\mathrm{c}}$ siècles)

Jiangxi tongzhi 江西通志, SKQS.

Xiuning xianzhi 修寧縣志, Hefei, Anhui jiaoyu chubanshe, 1990.

Xin Tangshu, voir OuYang Xiu. 
XU Chengyao 許承堯 (1874-1946)

2001 Sheshi xiantan 翊事閑談, dans le «Anhui guji congshu », Hefei, Huangshan shushe.

Xu Song 徐松 (Qing)

1957 Song huiyao jikao 宋會要輯稿, Beijing, Zhonghua shuju; édition en ligne http://songhuiyao.hul.harvard.edu.ezp-prod 1.hul.harvard.edu/ [abrégé SHY].

YE Mengde 葉夢德 (1077-1148)

Bishu luhua 避暑録話, SKQS.

Shilin yanyu 石林燕語, SKQS.

Yongle dadian, voir XIE Jin.

YONG Rong 永瑢 (1743-1790) \& JI Yun 紀旳 (1724-1805)

1986 《Wenyuan ge Siku quanshu » 文淵閣四庫全書, Taipei, Taiwan Shangwu yinshuguan, 1500 vol.

《Wenyuan ge Siku quanshu dianziban » 文淵閣四庫全書電子版, édition en ligne : http://10.34.2.9.ezp-prod1.hul.harvard.edu/scripts/skinet.dll?OnLoginPage [abrégé en SKQS]

ZHANG Bangji 張邦基 $\left(\mathrm{XII}^{\mathrm{e}}\right.$ siècle)

Mozhuang manlu 墨莊漫錄, SKQS.

ZHANG Peifang 張佩芳 (1732-1793) \& Liu Dakui 劉大槐 (1698-1779)

1975 Shexian zhi 銿縣志 (éd. de 1771), «Zhongguo fangzhi congshu : Huazhong difang ", Taipei, Chengwen chubanshe.

ZHANG Yanyuan 張彣遠 (813-879?)

Lidai minghua ji 歷代名畫記, SKQS.

ZHAO Ding 趙鼎 (1085-1147)

Zhongzhengde wenji 忠正德文集, SKQS.

ZHOU Jiazhou 周嘉冒 (1582-1658)

1939 Zhuanghuangzhi 裝㣴志, «Congshu jicheng chubian », Shanghai, Shangwu yinshuguan.

ZHU Mu 祝穆 $\left(\mathrm{XIII}^{\mathrm{e}}\right.$ siècle)

Fangyu shenglan 方輿勝覽, SKQS.

\section{Études et ouvrages}

Auteur(s) : collectif

1988 «Effets d'ordre dans la civilisation chinoise (rangements à l'œuvre, classifications implicites) ", Extrême-Orient, Extrême-Occident, n ${ }^{\circ} 10$.

1989 Huizhou diqu jianzhi 徽州地區簡志, s.l., Huangshan shushe.

2004 Huizhou wuqiancun 徽州五千村, Hefei, Huanghan shushe.

A Feng 阿風 \& ZHANG Guowang 張國旺

" Ming Longqing ben Xiuning Dunxi Jinshi zupu suoshou Song Yuan Ming gongwenshu kaoxi 》明龍慶本修寧《璫溪金氏族譜》所收宋元明公文書考析, texte inédit. 
Balazs, Étienne \& Hervouet, Yves

1978 A Sung Bibliography, Hong Kong, The Chinese University Press.

BAO Yilai 鮑義來

2005 Huizhou gongyi 徽州工㙯, 《Huizhou wenhua quanshu 》徽州文化全書, Hefei, Anhui renmin daxue chubanshe.

BARRETT, T.H.

2011 "The Women Who Invented Notepaper: Towards a Comparative Historiography of Paper and Print ", Journal of the Royal Asiatic Society, series 3, 21/2, p. 199-210.

BisCHOFF, F.A.

1963 La forêt des pinceaux. Étude sur l'Académie du Han-lin sous la Dynastie des T'ang et traduction du Han lin tche, Bibliothèque de l'Institut des hautes études chinoises, vol. XVII, Paris, PUF.

BROOK, Tymothy

2005 The Chinese State in Ming Society, London, Routledge Curzon, 2005.

Bussottr, Michela

1997 Gravures de l'école de Hui. Étude du livre illustré chinois de la fin du XVI siècle au milieu du XVIr siècle, Paris, EPHE, thèse de doctorat, 1997, surtout p. 30-53.

2005 «Note sulla carta prodotta nel Sud dell'Anhui durante le dinastie Song e Yuan », dans Maurizio Scarpari \& Tiziana Lippiello (éd.), Scritti in onore di Lionello Lanciotti per l'ottantesimo compleanno, Venezia, Libreria Cafoscarina, p. 181-189.

2012 "Compiler, éditer, illustrer : les monographies du district de Xiuning ", BEFEO 95-96, 2008-2010 [2012], p. 213-290.

2014 "Le papier de Xuanzhou, quelques sources historiques ", intervention à la journée d'études $D^{\prime}$ 'Est en Ouest : relations bilatérales autour du papier entre l'Extrême-Orient et l'Occident organisée par l'HiCSA et l'INP, 10 octobre 2014 à l'INHA, Paris.

Bussotr, Michela \& B^o Guoqiang 魫國強

"Les généalogies de Huizhou » (catalogue des généalogies de Huizhou conservées

à la Bibliothèque nationale de Chine, département des livres rares et des collections spéciales, http://www.efeo.fr/base_huizhou/).

CARTER, Thomas Francis

1955 The Invention of Printing in China and Its Spread Westward, L. Carrington Goodricu (éd.), $2^{\mathfrak{c}}$ éd., New York, Ronald Press.

Cen Deling

2013 «Le papier xuan dans la restauration des peintures et calligraphies chinoises », Arts Asiatiques 68, p. 87-90.

CHANG Bide 昌彼得 et al.

1977 Songren zhuanji ziliao suoyin 宋人傳記資料索引, Taipei, Dingwen shuju.

CHEN Gang 陳剛

2012 《Inoue Nabunasa yu Qingguo zhizhifa »井上陳政與清國制紙法, Shilin 史林, 3, p. 128-132. 
CHFN Qi 陳琪

2010 《Qingmo Huizhou Qimen Zhuyuan Chenshi zongpu wenshu 》清末徽州礿 門竹源陳氏宗譜文書, Faguo hanxue 法國漢學, 13, p. 332-358.

CHEN Qiyu 陳基余 et al. (éd.)

2000 Anhui wenhua shi 安徽文化史, Nanjing, Nanjing daxue chubanshe.

CHEN Rui 陳瑞

2009 《Yuandai Huizhou lu de shougong ye " 元代徽州路的手工业, Anhui daxue xuebao 安徽大學學報, 33/1, p. 101-107.

CHEN Zhichao 陳智超

1995 Jiekai Song huiyao zhimi 解開《宋會要》之謎, Beijing, Shehui kexue wenxian chubanshe.

2001 Meiguo Hafu daxue Hafu yanjing tushuguan cang Mingdai Huizhou Fangshi qinyou shouzha qibai tongkao shi (美國哈佛大學哈佛燕京圖書館藏明代 徽州方氏親友手札七百通考釋), Hefei, Anhui daxue chubanshe.

Снü Ch'ing-yuan

1956 "Gouvernement artisans of the Yuan Dynasty », dans E-tu ZeN \& John DE FrAncis, Chinese Social History: translations of selected studies, Washington, American council of learned societies, p. 234-246.

Daniels, Christian \& MenzIes, Nicholas Kay

1996 Agro-industries: Sugarcane technology, Forestry, dans Joseph NeEdHam (éd.), "Science \& civilisation in China », vol. 6, part 3, Cambridge, Cambridge University Press.

DrĖGF, Jean-Pierre

1994 Chine Bambou Papier, Paris, Institut de France - Imprimerie de l'Indre.

2014 "Les controverses concernant les origines du papier en Chine », intervention à la journée d'études D'Est en Ouest : relations bilatérales autour du papier entre l'Extrême-Orient et l'Occident organisée par l'HiCSA et l'INP, 10 octobre 2014 à l'INHA, Paris.

FARQUHAR, David M.

1990 The Government of China under Mongolian Rule. A Reference Guide, Stuttgart, Franz Steiner, Verlag GmbH.

FENG Qicai 冯䩀才 (éd.) et al.

2007 Zhongguo muban nianhuajicheng: Tandoujuan 中國木版年畫集成滩头卷, Beijing, Zhonghua shuju.

FĖVRE, Francine \& MétalLié, Georges

2005 Dictionnaire Ricci des plantes de Chine, Paris, éditions du Cerf.

Foucault, Michel

1991 Les mots et les choses, une archéologie des sciences humaines, Paris, Gallimard.

Franke, Herbert \& Twitchett, Denis C.

1994 Alien Regimes and Border States 907-1368, The Cambridge history of China, vol. 6, New York, Cambridge Univesity Press.

Fuchs, Walter

1946 "The "Mongol Atlas" of China by Chu Ssu-pen and the Kuang-yii-t'u, with 48 fac-simile maps dating from about $1555 »$, Monumenta Serica, monograph VIII. 
HARGET, James M.

1989 On the road in twelfth century China: The travel diaries of Fan Chengda (1126-1193), Stuttgart, Steiner Verl. Wiesbaden.

Hou Ching-ling

1975 Monnaies d'offrande et la notion de trésorerie dans la religion chinoise, Paris, Collège de France, Institut des hautes études chinoises.

Hu Huakai 胡化凱 \& ZHANG Binglun 㢭秉倫

2005 Huizhou keji 徽州科技, “ Huizhou wenhua quanshu », Hefei, Anhui renmin daxue chubanshe.

Hucker, Charles Oscar

1985 A Dictionary of Official titles in Imperial China, Taipei, SMC Publishing INC.

Lamouroux, Christian

2003 Fiscalité, comptes publics et politiques financières dans la Chine des Song, Paris, Collège de France, Institut des hautes études chinoises.

2013 " À travers le Miroir, Une controverse politique sous les Song ", Études chinoises XXXII/1, p. 129-151.

"L'émergence du papier monétaire dans l'empire chinois $\left(\mathrm{XI}^{\mathrm{e}}-\mathrm{XII}^{\mathrm{e}}\right.$ siècles $)$ », synthèse inédite.

ORSENNA, Érik

2013 Sur la route du papier. Petit précis de mondialisation III, Paris, Stock.

PAN Jixing 潘吉星

1998 Zaozhi yu yinshua juan 造紙與印刷卷, dans «Zhongguo kexue jishu shi » 中國科學技術史, Beijing, Kexue chubanshe.

2011 "Review on the debate of paper history during recent 30 years in China ", Journal of the International Association of Paper Historians, vol. 15/2, p. 6-12.

Pauthier, Guillaume (1801-1873) (éd.)

1865 Le livre de Marco Polo, citoyen de Venise... par M. G. Pauthier, Paris, Typographie de Firmin Didot frères imprimeurs de l'Institut de France.

Polastron, Lucien

1999 Le papier. 2000 ans d'histoire et de savoir-faire, Paris, Imprimerie nationale.

2010 Le trésor des lettrés, Paris, Imprimerie nationale.

$\mathrm{Pu} X \mathrm{Xia}$ 蒲霞

2010 《Zhu Tong Xin'an zhi de jiazhi ji qi liyong》朱同《新安志》的價值及其 利用, Huixue 榇學, 6, p. 211-219.

RatCHNEVSKY, Paul et al.

1972-1977 Un code des Yuan, Paris, PUF.

1985 Un code des Yuan, Paris, Collège de France, Institut des hautes études chinoises.

Rossabi, Morris

1988 Khubilai Khan His Life and Times, London / Berkeley, University of California Press.

SABBAN, Françoise

1994 "L'industrie sucrière, le moulin à sucre et les relations sino-portugaises aux $\mathrm{XVI}^{\mathrm{e}}$-XVIII" siècles », Annales. Histoire, Sciences Sociales 48, p. 817-861. 
SCHÄFER, Dagmar (éd.)

2012 Cultures of Knowledge: Technology in Chinese History, Leiden, Brill.

Schmitt, Camille

2011 La médecine de l'art. Conservation des calligraphies et peintures chinoises en rouleaux à partir des textes de la dynastie Ming (1368-1644), Paris, Picquier.

SCHURMANN, H. F.

1956 Economic Structure of the Yuan Dynasty, Translation of Chapter 93 and 94 of the "Yuanshi », Cambridge - Massachusetts, Harvard University Press.

Shanghai TuShuguan 上海圖書館 (éd.)

2008 Zhongguo jiapu zongmu 中國家譜總目, Shanghai, Shanghai guji chubanshe.

VON STROMER, Wolfgang

2000 Le mystère Gutenberg de Tourfan à Karlstein. Les origines chinoises de l'imprimerie, Genève, Slatkine.

TSIEN Tsuen-Hsuin

1985 Paper and Printing, dans Joseph Needham (éd.), « Science and Civilisation in China », vol. 5, part 1, Cambridge, Cambridge University Press.

Vogel, Hans Ulrich

2013 Marco Polo Was in China, New Evidence from Currencies, Salts and Revenues, Leiden / Boston, Brill.

WeI Huaxian 魏華仙

2006 Songdai silei wupin de shengchan he xiaofei yanjiu 宋代四類物品的生產和 消費研究, Chengdu, Sichuan chuban tuanji - Sichuan kexue jishu chubanshe.

WILL, Pierre-Étienne

1992 «Chinese Local Gazetteers: An Historical and Practical Introduction », Notes de recherches $d u$ centre Chine, $\mathrm{n}^{\circ} 3$, Centre de Recherches et de Documentation sur la Chine contemporaine.

XIAO Jianxin 蕭建新

2006 《Lun Xin'an zhi keben de xitong he jiazhi 》論《新安志》刻本的系統和 價值, Huazhong keji daxue xuebao (shekeban) 华中科技大学学报（社科 版) , 2006/3.

2008 voir Luo Yuan.

ZHAI Tunjian

2015 « Étude comparative des monographies de la préfecture de Hui et du district de She », dans M. BussotTI \& J.-P. Drège (éd.), Imprimer sans profit, Paris / Genève, EPHE / Droz, p. 289-337.

ZHANG Binglun 張秉倫, FAN Jialu 樊嘉祿 \& FANG Xiaoyang 方曉陽

2005 Zaozhi yu yinshua 造紙與印刷, «Zhongguo chuantong gongyi quanji » 中國 傳統工藝全集, Zhengzhou, Daxiang chubanshe.

ZHANG Yingpin 張英聘

2005 Mingdai Nanzhili fangzhi yanjiu 明代南直隸方志研究, Beijing, Shehui kexue wenxuan chubanshe. 